\title{
ASYMPTOTIC STABILITY OF SHOCK PROFILES AND RAREFACTION WAVES UNDER PERIODIC PERTURBATIONS FOR 1-D CONVEX SCALAR VISCOUS CONSERVATION LAWS
}

\author{
ZHOUPING XIN, QIAN YUAN, AND YUAN YUAN
}

\begin{abstract}
This paper studies the asymptotic stability of shock profiles and rarefaction waves under space-periodic perturbations for one-dimensional convex scalar viscous conservation laws. For the shock profile, we show that the solution approaches the background shock profile with a constant shift in the $L^{\infty}(\mathbb{R})$ norm at exponential rates. The new phenomena contrasting to the case of localized perturbations is that the constant shift cannot be determined by the initial excessive mass in general, which indicates that the periodic oscillations at infinities make contributions to this shift. And the vanishing viscosity limit for the shift is also shown. The key elements of the poof consist of the construction of an ansatz which tends to two periodic solutions as $x \rightarrow \pm \infty$, respectively, and the anti-derivative variable argument, and an elaborate use of the maximum principle. For the rarefaction wave, we also show the stability in the $L^{\infty}(\mathbb{R})$ norm.
\end{abstract}

\section{Contents}

1. Introduction and main results

2. Preliminaries and ansatz 5

3. Stability of shock profiles 8

3.1. Shift function 8

3.2. Decay to ansatz 14

3.3. More results for Burgers' equation 27

3.4. An example of non-zero shift 31

3.5. Vanishing viscosity limit for the shift 32

4. Stability of rarefaction waves 35

$\begin{array}{lll}\text { Appendix A. } & \text { Proof of Lemma 2.1 } & 37\end{array}$

Appendix B. Proof of Lemma 2.2 40

Appendix C. Proof of Lemma 2.4 41

References $\quad 42$

\section{INTRODUCTION AND MAIN RESULTS}

We consider the Cauchy problem for one-dimensional convex scalar viscous conservation laws

$$
\begin{array}{rlrl}
\partial_{t} u^{\nu}+\partial_{x} f\left(u^{\nu}\right) & =\nu \partial_{x}^{2} u^{\nu}, & & x \in \mathbb{R}, t>0, \\
u^{\nu}(x, 0) & =u_{0}(x), \quad x \in \mathbb{R},
\end{array}
$$

This research is partially supported by Zheng Ge Ru Foundation, Hong Kong RGC Earmarked Research Grants, CUHK-14300917, CUHK-14305315, and CUHK-14302917.

The research of Yuan Yuan is also supported by the Start-up Research Grant of South China Normal University (8S0328). 
where $u^{\nu}(x, t) \in \mathbb{R}$ is the unknown, the flux $f$ is smooth and strictly convex, and $\nu \in(0,1]$ denotes the viscosity. This paper is concerned with the asymptotic behavior of the solution $u^{\nu}(x, t)$ to $(1.1),(1.2)$ with $u_{0}(x) \in L^{\infty}(\mathbb{R})$ satisfying

$$
\left\{\begin{array}{l}
\left|u_{0}(x)-\bar{u}_{l}-w_{0 l}(x)\right| \leqslant C_{0} e^{\beta_{0} x}, \\
\left|u_{0}(x)-\bar{u}_{r}-w_{0 r}(x)\right| \leqslant C_{0} e^{-\beta_{0} x},
\end{array} \quad x \in \mathbb{R},\right.
$$

where $\bar{u}_{l}, \bar{u}_{r}, C_{0}>0$ and $\beta_{0}>0$ are constants, and $w_{0 l}, w_{0 r} \in L^{\infty}(\mathbb{R})$ are two arbitrary periodic functions.

It is well known from $[28,20]$ that for $\nu>0$, the equation (1.1) generates a semigroup $\left\{S_{t}^{\nu}: L^{\infty}(\mathbb{R}) \rightarrow L^{\infty}(\mathbb{R}) ; t \geqslant 0\right\}$ to ensure that, for any initial data $u_{0} \in L^{\infty}(\mathbb{R})$, the function $u^{\nu}(x, t):=S_{t}^{\nu} u_{0}$ is the unique bounded solution to (1.1), (1.2), which is smooth for $t>0$, and satisfies the initial condition in the weak sense: for any continuous functions $\varphi(x, t)$ compactly supported in $\mathbb{R} \times[0,+\infty)$, there holds

$$
\int_{\mathbb{R}}\left[\varphi(x, t) u^{\nu}(x, t)-\varphi(x, 0) u_{0}(x)\right] d x \rightarrow 0 \quad \text { as } t \rightarrow 0 .
$$

The semi-group $S_{t}^{\nu}$ satisfies the following classical Co-properties:

- (Comparison) If $u_{0}, v_{0} \in L^{\infty}(\mathbb{R})$ and $u_{0} \leqslant v_{0}$ almost everywhere, then $S_{t}^{\nu} u_{0} \leqslant$ $S_{t}^{\nu} v_{0}$ for any $x \in \mathbb{R}, t>0$.

- (Contraction) If $u_{0}, v_{0} \in L^{\infty}(\mathbb{R})$ and $u_{0}-v_{0} \in L^{1}(\mathbb{R})$, then $S_{t}^{\nu} u_{0}-S_{t}^{\nu} v_{0} \in L^{1}(\mathbb{R})$ and $\left\|S_{t}^{\nu} u_{0}-S_{t}^{\nu} v_{0}\right\|_{L^{1}(\mathbb{R})}$ is non-increasing with respect to $t$.

- (Conservation) If $u_{0}, v_{0} \in L^{\infty}(\mathbb{R})$ and $u_{0}-v_{0} \in L^{1}(\mathbb{R})$, then

$$
\int_{\mathbb{R}}\left(S_{t}^{\nu} u_{0}-S_{t}^{\nu} v_{0}\right) d x=\int_{\mathbb{R}}\left(u_{0}-v_{0}\right) d x \quad \forall t \geqslant 0 .
$$

Moreover, there exists a constant $E>0$, depending only on $f$ and $\left\|u_{0}\right\|_{L^{\infty}}$, such that

$$
\partial_{x} u^{\nu}(x, t) \leqslant \frac{E}{t} \quad \forall x \in \mathbb{R}, t>0 .
$$

Shocks and rarefaction waves are most important nonlinear solutions to conservation laws. A viscous shock profile $\phi^{\nu}(x-s t)$ is a classical traveling wave solution to the viscous conservation law (1.1), solving the problem:

$$
\left\{\begin{array}{l}
\nu\left(\phi^{\nu}\right)^{\prime \prime}=f^{\prime}\left(\phi^{\nu}\right)\left(\phi^{\nu}\right)^{\prime}-s\left(\phi^{\nu}\right)^{\prime} \\
\lim _{x \rightarrow-\infty} \phi^{\nu}(x)=\bar{u}_{l}, \lim _{x \rightarrow+\infty} \phi^{\nu}(x)=\bar{u}_{r}
\end{array}\right.
$$

where $\bar{u}_{l}>\bar{u}_{r}$ and $s$ is the shock speed defined by the Rankine-Hugoniot condition:

$$
s=\frac{f\left(\bar{u}_{l}\right)-f\left(\bar{u}_{r}\right)}{\bar{u}_{l}-\bar{u}_{r}} .
$$

The existence of the shock profile follows from a simple phase plane analysis, and can also follow from the center-manifold theorem in Kopell-Howard [18]. For $\bar{u}_{l}<\bar{u}_{r}$, a centered rarefaction wave

$$
u^{R}(x, t)= \begin{cases}\bar{u}_{l}, & \frac{x}{t}<f^{\prime}\left(\bar{u}_{l}\right), \\ \left(f^{\prime}\right)^{-1}\left(\frac{x}{t}\right), & f^{\prime}\left(\bar{u}_{l}\right) \leqslant \frac{x}{t} \leqslant f^{\prime}\left(\bar{u}_{r}\right), \\ \bar{u}_{r}, & \frac{x}{t}>f^{\prime}\left(\bar{u}_{r}\right),\end{cases}
$$

is an entropy weak solution to the Riemann problem for the inviscid conservation law (1.1) with $\nu=0$. 
When the initial data $u_{0}$ approaches constant states as $|x| \rightarrow+\infty$, i.e. $w_{0 l}=$ $w_{0 r}=0$ in (1.3), the asymptotic behaviors of solutions to (1.1), (1.2) have been studied widely so far. The pioneering work of Hopf [14] showed the $L^{\infty}$ stability of constants with a decay rate $1 / \sqrt{t}$, by using an explicit representation of the solution to the Burgers' equation. Later, Il'in-Olernik [16] applied the maximum principle on the anti-derivative variables to prove the $L^{\infty}$ stability of constants, shocks and rarefaction waves for general convex conservation laws. Moreover, Freistühler-Serre [5] combined a lap number argument and maximum principle to prove the $L^{1}$ stability of viscous shock profiles. For more results, we refer to [27, 11, 15] for the onedimensional scalar case, $[9,32,12,13,17]$ for the multi-dimensional scalar case, and $[26,8,23,24,31,25,29]$ for the important one-dimensional system case.

For the initial data which keeps oscillating at infinities, Lax [22] was the first one to study the periodic data. He showed that the entropy periodic solutions to inviscid scalar conservation laws approach their constant averages in the $L^{\infty}$ norm at algebraic rates. And then Glimm-Lax [7] and Dafermos [1] used the generalized characteristics to extend the results to some $2 \times 2$ systems. Besides the constants, Xin-Yuan-Yuan [33] and Yuan-Yuan [34] proved the $L^{\infty}$ stability of shocks and rarefaction waves under periodic perturbations for the scalar inviscid conservation laws, by using the generalized characteristics. One can also see [3, 4] for the $L^{1}$ stability of the stationary viscous "shock profile" which connects two periodic functions as end states, where the flux in the equation (1.1) is $f(x, u)$ that is periodic with respect to $x$.

In this paper, we deal with the initial data (1.3), where $w_{0 l}, w_{0 r}$ are arbitrary periodic functions, in order to see how the initial oscillations at infinities influence the stability of these two nonlinear waves in the viscous case. Throughout this paper, we let $\phi(x)$ denote any fixed shock profile solving (1.5) with $\nu=1$, and then for any $\nu>0$, we define

$$
\phi^{\nu}(x):=\phi\left(\frac{x}{\nu}\right) .
$$

Then $\phi^{\nu}$ solves (1.5) for any $\nu>0$ and tends to the inviscid Lax-shock

$$
u^{S}(x, t)= \begin{cases}\bar{u}_{l}, & x<s t, \\ \bar{u}_{r}, & x>s t,\end{cases}
$$

almost everywhere as the viscosity $\nu \rightarrow 0+$. With the constants $\bar{u}_{l}, \bar{u}_{r}$ and periodic perturbations $w_{0 l}, w_{0 r} \in L^{\infty}(\mathbb{R})$ in $(1.3)$, we let $u_{l}^{\nu}, u_{r}^{\nu}$ denote the periodic solutions to (1.1) with the respective initial data

$$
u_{l}^{\nu}(x, 0)=\bar{u}_{l}+w_{0 l}(x), \quad u_{r}^{\nu}(x, 0)=\bar{u}_{r}+w_{0 r}(x) .
$$

Now the main results of this paper are stated as follows:

Theorem 1.1. Assume that $\nu>0$ and the initial data $u_{0} \in L^{\infty}(\mathbb{R})$ satisfies (1.3) with $\bar{u}_{l}>\bar{u}_{r}$ and the periodic functions $w_{0 l}, w_{0 r} \in L^{\infty}$ with the respective periods $p_{l}, p_{r}>0$, satisfying

$$
\frac{1}{p_{l}} \int_{0}^{p_{l}} w_{0 l}(x) d x=\frac{1}{p_{r}} \int_{0}^{p_{r}} w_{0 r}(x) d x=0 .
$$

Then the unique bounded solution $u^{\nu}$ to (1.1), (1.2) satisfies

$$
\sup _{x \in \mathbb{R}}\left|u^{\nu}(x, t)-\phi^{\nu}\left(x-s t-X_{\infty}^{\nu}\right)\right| \leqslant C e^{-\mu t}, \quad t>0,
$$


with the constant shift $X_{\infty}^{\nu}=\frac{1}{\bar{u}_{l}-\bar{u}_{r}}\left(X_{\infty, 1}^{\nu}+X_{\infty, 2}^{\nu}\right)$ given by

$$
\begin{aligned}
X_{\infty, 1}^{\nu}= & \int_{-\infty}^{0}\left(u_{0}-\phi^{\nu}-w_{0 l}\right)(x) d x+\int_{0}^{+\infty}\left(u_{0}-\phi^{\nu}-w_{0 r}\right)(x) d x \\
X_{\infty, 2}^{\nu}= & \int_{0}^{+\infty} \frac{1}{p_{l}} \int_{0}^{p_{l}}\left[f\left(u_{l}^{\nu}(x, t)\right)-f\left(\bar{u}_{l}\right)\right] d x d t-\frac{1}{p_{l}} \int_{0}^{p_{l}} \int_{0}^{x} w_{0 l}(y) d y d x \\
& -\int_{0}^{+\infty} \frac{1}{p_{r}} \int_{0}^{p_{r}}\left[f\left(u_{r}^{\nu}(x, t)\right)-f\left(\bar{u}_{r}\right)\right] d x d t+\frac{1}{p_{r}} \int_{0}^{p_{r}} \int_{0}^{x} w_{0 r}(y) d y d x .
\end{aligned}
$$

Theorem 1.1 shows that in contrast to the case of localized perturbations, besides the localized part of the initial perturbation, the periodic oscillations at infinities generate another shift $X_{\infty, 2}^{\nu}$ to the background viscous shock profile. The next theorem shows that this shift is non-zero in general even in the case the periods of the perturbations at $x= \pm \infty$ are the same.

Theorem 1.2. Under the assumptions of Theorem 1.1, if $w_{0 l}=w_{0 r}=w_{0}$, the constant $X_{\infty, 2}^{\nu}$ defined in (1.11) may be non-zero in general. More precisely,

(1) for the Burgers' equation, i.e. $f(u)=u^{2} / 2$, it holds that $X_{\infty, 2}^{\nu}=0$;

(2) for any periodic perturbation $w_{0}$ with zero average, if

$$
0<\left\|w_{0}\right\|_{L^{\infty}(\mathbb{R})}<\left(\bar{u}_{l}-\bar{u}_{r}\right) / 2,
$$

there exists a smooth and strictly convex flux $f$ such that $X_{\infty, 2}^{\nu} \neq 0$.

However, for the inviscid conservation law (1.1) with $\nu=0$, it is shown in [33] that if $w_{0 l}=w_{0 r}$, the entropy solution $u^{0}$ tends to the background shock $u^{S}$ with no shift, i.e. $X_{\infty, 2}^{0}=0$ in this case. The vanishing viscosity limit for the shift $X_{\infty, 2}^{\nu}$ is presented in the next theorem, which agrees with the results in [33, 34].

Theorem 1.3. Under the assumptions of Theorem 1.1, as the viscosity $\nu \rightarrow 0+$,

$$
X_{\infty, 2}^{\nu} \rightarrow X_{\infty, 2}^{0}:=-\min _{x \in \mathbb{R}} \int_{0}^{x} w_{0 l}(y) d y+\min _{x \in \mathbb{R}} \int_{0}^{x} w_{0 r}(y) d y .
$$

Furthermore, if both $w_{0 l}$ and $w_{0 r}$ have bounded total variations on their respective periodic domains, there exists a constant $C>0$, independent of the viscosity $\nu$, such that

$$
\left|X_{\infty, 2}^{\nu}-X_{\infty, 2}^{0}\right| \leqslant C \nu^{1 / 5} .
$$

At last, we state the result for rarefaction waves.

Theorem 1.4. Assume that $\nu>0$ and the initial data $u_{0}$ satisfies (1.3) with $\bar{u}_{l}<$ $\bar{u}_{r}$ and the periodic functions $w_{0 l}, w_{0 r} \in L^{\infty}$ with the respective periods $p_{l}, p_{r}>0$, satisfying (1.8). Then the unique bounded solution $u^{\nu}$ to (1.1), (1.2) satisfies

$$
\sup _{x \in \mathbb{R}}\left|u^{\nu}(x, t)-u^{R}(x, t)\right| \rightarrow 0 \quad \text { as } t \rightarrow \infty .
$$

Consequently, different from the localized perturbations, i.e., the background shock can absorb all localized perturbations on the two sides and finally tends to itself with no shift (if the perturbation has zero mass), the periodic perturbations at infinities produce infinite perturbations onto the background shock, ended up with a constant shift, which cannot be determined explicitly by the initial perturbations in the viscous case. 
The main difficulty to prove Theorem 1.1 is that the perturbation $u^{\nu}-\phi^{\nu}$ is not integrable anymore, which makes it difficult to use an anti-derivative argument as before that plays an important role in the previous study of the stability under localized perturbations. In fact, if one considers the equation of the perturbation, the coefficient of the zero-order term is $f^{\prime \prime}\left(\phi^{\nu}\right)\left(\phi^{\nu}\right)^{\prime}<0$, which makes it harder to use either the maximum principle or the energy method. One of the key elements of our proof is that we find an ansatz $\psi_{X^{\nu}(t)}^{\nu}(x, t)$, where $X^{\nu}(t)$ is a shift function (see (2.15)) such that the difference $u^{\nu}-\psi_{X^{\nu}(t)}^{\nu}$ is integrable and has zero mass for large time. Therefore, it is plausible to study the equation of the anti-derivative variable of the difference $u^{\nu}-\psi_{X^{\nu}(t)}^{\nu}$ (for the Burgers' equation, the ansatz actually coincides with the solution $u^{\nu}$ at an arithmetic sequence of time $\left\{t_{k}\right\}$; see Proposition 2.8). We show that the limit of $X^{\nu}(t)-s t$ as $t \rightarrow+\infty$ is actually the constant shift $X_{\infty}^{\nu}$ in Theorem 1.1. And although the ansatz $\psi_{X^{\nu}(t)}^{\nu}$ is not a solution to (1.1), the error (see (2.10)) decays exponentially both in space and in time. Then following the idea of Il'in-Oleinnik [16], one can construct auxiliary functions and use the maximum principle to obtain our main results.

\section{Preliminaries AND ANSATZ}

We first present some useful lemmas and introduce some notations, and then construct the ansatz. Important properties of the ansatz will be stated as propositions, which will be proved in the rest of the paper. In the end of this section, we outline the organizations of the proof.

Lemma 2.1. Assume that $u_{0}(x) \in L^{\infty}(\mathbb{R})$ is periodic with period $p>0$ and average $\bar{u}=\frac{1}{p} \int_{0}^{p} u_{0}(x) d x$. Then there exists a constant $\alpha>0$, depending only on $p$ and $\nu$, such that for any integers $k, l \geqslant 0$, the periodic solution $u^{\nu}(x, t)$ to (1.1),(1.2) satisfies that

$$
\left\|\partial_{t}^{k} \partial_{x}^{l}\left(u^{\nu}-\bar{u}\right)\right\|_{L^{\infty}(\mathbb{R})} \leqslant C e^{-\alpha t}, \quad t \geqslant 1,
$$

where $C>0$ is independent of time $t$.

The proof of Lemma 2.1 can be obtained by standard energy estimates and the Poincaré inequality, which is given in the Appendix A. Thus, there exists an $\alpha>0$, depending on $p_{l}, p_{r}$ and $\nu$, such that $u_{l}^{\nu}$ and $u_{r}^{\nu}$ satisfy (2.1) with $\bar{u}=\bar{u}_{l}$ and $\bar{u}_{r}$, respectively.

Lemma 2.2. Assume that $u_{0}, \tilde{u}_{0} \in L^{\infty}(\mathbb{R})$ and there exist constants $C>0$ and $\delta \in \mathbb{R}$ such that

$$
\left|u_{0}(x)-\tilde{u}_{0}(x)\right| \leqslant C e^{\delta x}, \quad x \in \mathbb{R} .
$$

Then it holds that

$$
\left|S_{t}^{\nu} u_{0}-S_{t}^{\nu} \tilde{u}_{0}\right| \leqslant C(t) e^{\delta x}, \quad x \in \mathbb{R}, t>0,
$$

where the constant $C(t)>0$ is bounded on any compact subset of $[0,+\infty)$.

The proof of Lemma 2.2, based on approximate solutions solving linear parabolic equations, is given in the Appendix B.

For the periodic solutions $u_{l}^{\nu}$ and $u_{r}^{\nu}$ defined in (1.7), the following result can follow from Lemma 2.2. 
Lemma 2.3. Assume that the initial data $u_{0} \in L^{\infty}(\mathbb{R})$ satisfies (1.3). Then the unique solution $u^{\nu}$ to (1.1), (1.2) satisfies that

$$
\left\{\begin{array}{l}
\left|u^{\nu}(x, t)-u_{l}^{\nu}(x, t)\right| \leqslant C(t) e^{\beta_{0} x}, \\
\left|u^{\nu}(x, t)-u_{r}^{\nu}(x, t)\right| \leqslant C(t) e^{-\beta_{0} x},
\end{array} \quad x \in \mathbb{R}, t>0,\right.
$$

where $C(t)$ is bounded on any compact subset of $[0,+\infty)$.

To prove Theorem 1.1, we now construct the ansatz. For the shock profile $\phi^{\nu}$ defined in (1.6), we first define the function:

$$
g^{\nu}(x):=\frac{\phi^{\nu}(x)-\bar{u}_{r}}{\bar{u}_{l}-\bar{u}_{r}}, \quad x \in \mathbb{R} .
$$

Lemma 2.4. The function $g^{\nu} \in C^{\infty}(\mathbb{R})$ satisfies that

(i) there exist positive constants $\beta_{1}$ and $\beta_{2}$, depending on $\nu, f, \bar{u}_{l}$ and $\bar{u}_{r}$, such that

$$
\beta_{1} \leqslant \frac{-\left(g^{\nu}\right)^{\prime}(x)}{\left(\bar{u}_{l}-\bar{u}_{r}\right) g^{\nu}(x)\left(1-g^{\nu}(x)\right)} \leqslant \beta_{2}, \quad x \in \mathbb{R}
$$

(ii) with the inequality (2.4), there exists a constant $C>0$, depending on $\nu, f, \bar{u}_{l}$ and $\bar{u}_{r}$, such that

$$
\begin{array}{ll}
\frac{1}{C} e^{-\beta_{2} x} \leqslant g^{\nu}(x) \leqslant C e^{-\beta_{1} x}, & x>0, \\
\frac{1}{C} e^{\beta_{2} x} \leqslant 1-g^{\nu}(x) \leqslant C e^{\beta_{1} x}, & x<0, \\
\frac{1}{C} e^{-\beta_{2}|x|} \leqslant-\left(g^{\nu}\right)^{\prime}(x) \leqslant C e^{-\beta_{1}|x|}, & x \in \mathbb{R} .
\end{array}
$$

The proof of Lemma 2.4 can be found in [10]. And we will give a simplified proof for the scalar viscous conservation laws in the Appendix C. For convenience, in the following part of this paper we define

$$
\beta:=\min \left\{\beta_{0}, \beta_{1}, \beta_{2}\right\}>0 .
$$

Then for any $C^{1}$ curve $\xi(t):[0,+\infty) \rightarrow \mathbb{R}$, we set

$$
g_{\xi}^{\nu}(x):=g^{\nu}(x-\xi(t)), \quad x \in \mathbb{R}, t \geqslant 0,
$$

with the derivatives:

$$
\left(g_{\xi}^{\nu}\right)^{(k)}(x):=\left(g^{\nu}\right)^{(k)}(x-\xi(t)), \quad k \geqslant 1 .
$$

Motivated by Lemma 2.3 and the formula of the viscous shock profile

$$
\phi_{\xi}^{\nu}(x):=\phi^{\nu}(x-\xi(t))=\bar{u}_{l} g_{\xi}^{\nu}(x)+\bar{u}_{r}\left(1-g_{\xi}^{\nu}(x)\right),
$$

we construct the ansatz as

$$
\psi_{\xi}^{\nu}(x, t):=u_{l}^{\nu}(x, t) g_{\xi}^{\nu}(x)+u_{r}^{\nu}(x, t)\left(1-g_{\xi}^{\nu}(x)\right) .
$$

It is noted that the shift $\xi(t)$ appears only in $g^{\nu}$. Thus $\psi_{\xi}^{\nu}$ satisfies

$$
\partial_{t} \psi_{\xi}^{\nu}+\partial_{x} f\left(\psi_{\xi}^{\nu}\right)-\nu \partial_{x}^{2} \psi_{\xi}^{\nu}=h_{\xi}^{\nu},
$$

where the source term $h_{\xi}^{\nu}$ is

$$
\begin{aligned}
h_{\xi}^{\nu}= & \partial_{x} f\left(\psi_{\xi}^{\nu}\right)-\partial_{x} f\left(u_{l}^{\nu}\right) g_{\xi}^{\nu}-\partial_{x} f\left(u_{r}^{\nu}\right)\left(1-g_{\xi}^{\nu}\right)-2 \nu \partial_{x}\left(u_{l}^{\nu}-u_{r}^{\nu}\right)\left(g_{\xi}^{\nu}\right)^{\prime} \\
& -\left(u_{l}^{\nu}-u_{r}^{\nu}\right)\left(\left(g_{\xi}^{\nu}\right)^{\prime} \xi^{\prime}+\nu\left(g_{\xi}^{\nu}\right)^{\prime \prime}\right),
\end{aligned}
$$


which can be rewritten as

$$
\begin{aligned}
h_{\xi}^{\nu}= & \partial_{x}\left[\left(f\left(\psi_{\xi}^{\nu}\right)-f\left(u_{l}^{\nu}\right)\right) g_{\xi}^{\nu}+\left(f\left(\psi_{\xi}^{\nu}\right)-f\left(u_{r}^{\nu}\right)\right)\left(1-g_{\xi}^{\nu}\right)-2 \nu\left(u_{l}^{\nu}-u_{r}^{\nu}\right)\left(g_{\xi}^{\nu}\right)^{\prime}\right] \\
& +\left(f\left(u_{l}^{\nu}\right)-f\left(u_{r}^{\nu}\right)\right)\left(g_{\xi}^{\nu}\right)^{\prime}-\left(u_{l}^{\nu}-u_{r}^{\nu}\right)\left(g_{\xi}^{\nu}\right)^{\prime} \xi^{\prime}+\nu\left(u_{l}^{\nu}-u_{r}^{\nu}\right)\left(g_{\xi}^{\nu}\right)^{\prime \prime} .
\end{aligned}
$$

This, together with the fact $\nu\left(g_{\xi}^{\nu}\right)^{\prime \prime}=f^{\prime}\left(\phi_{\xi}^{\nu}\right)\left(g_{\xi}^{\nu}\right)^{\prime}-s\left(g_{\xi}^{\nu}\right)^{\prime}$, yields that

$$
\begin{aligned}
h_{\xi}^{\nu}= & \left(f^{\prime}\left(\psi_{\xi}^{\nu}\right)-f^{\prime}\left(u_{l}^{\nu}\right)\right) \partial_{x} u_{l}^{\nu} g_{\xi}^{\nu}+\left(f^{\prime}\left(\psi_{\xi}^{\nu}\right)-f^{\prime}\left(u_{r}^{\nu}\right)\right) \partial_{x} u_{r}^{\nu}\left(1-g_{\xi}^{\nu}\right) \\
& -2 \nu \partial_{x}\left(u_{l}^{\nu}-u_{r}^{\nu}\right)\left(g_{\xi}^{\nu}\right)^{\prime}-\left(u_{l}^{\nu}-u_{r}^{\nu}\right)\left(\xi^{\prime}(t)-s+f^{\prime}\left(\phi_{\xi}^{\nu}\right)-f^{\prime}\left(\psi_{\xi}^{\nu}\right)\right)\left(g_{\xi}^{\nu}\right)^{\prime} .
\end{aligned}
$$

The formulas (2.11) and (2.12) will be used later.

The ansatz $\psi_{\xi}^{\nu}$ is expected to satisfy $\int_{\mathbb{R}}\left(u^{\nu}-\psi_{\xi}^{\nu}\right)(x, t) d x \equiv 0$, so that the antiderivative variable can vanish at both infinities $|x| \rightarrow \infty$. Under the assumptions of Theorem 1.1, it follows from Lemma 2.1 that there exists a large $T_{0}>0$ such that

$$
0<\frac{\bar{u}_{l}-\bar{u}_{r}}{2}<\left(u_{l}^{\nu}-u_{r}^{\nu}\right)(x, t)<2\left(\bar{u}_{l}-\bar{u}_{r}\right), \quad x \in \mathbb{R}, t \geqslant T_{0} .
$$

The time $T_{0}$ is chosen to guarantee $\int_{\mathbb{R}}\left(u_{l}^{\nu}-u_{r}^{\nu}\right)(x, t)\left(g_{\xi}^{\nu}\right)^{\prime} d x<0$ for all $t \geqslant T_{0}$. It is noted that the terms appearing in the square brackets of (2.11) vanish as $|x| \rightarrow \infty$. For the equation of the perturbation,

$$
\partial_{t}\left(u^{\nu}-\psi_{\xi}^{\nu}\right)+\partial_{x}\left(f\left(u^{\nu}\right)-f\left(\psi_{\xi}^{\nu}\right)\right)=\nu \partial_{x}^{2}\left(u^{\nu}-\psi_{\xi}^{\nu}\right)-h_{\xi}^{\nu},
$$

we aim to choose a curve $\xi=X^{\nu}(t)$ such that $\int_{\mathbb{R}} h_{X^{\nu}}^{\nu}(x, t) d x=0$. Integrating (2.11) with respect to $x$, we require that the curve $X^{\nu}(t)$ solves the problem:

$$
\left\{\begin{array}{l}
\left(X^{\nu}\right)^{\prime}(t)=F^{\nu}\left(X^{\nu}(t), t\right), \quad t>T_{0}, \\
X^{\nu}\left(T_{0}\right)=X_{0}^{\nu}
\end{array}\right.
$$

where

$$
F^{\nu}(\xi, t):=\frac{\int_{\mathbb{R}}\left[\nu\left(u_{l}^{\nu}-u_{r}^{\nu}\right)\left(g_{\xi}^{\nu}\right)^{\prime \prime}+\left(f\left(u_{l}^{\nu}\right)-f\left(u_{r}^{\nu}\right)\right)\left(g_{\xi}^{\nu}\right)^{\prime}\right] d x}{\int_{\mathbb{R}}\left(u_{l}^{\nu}-u_{r}^{\nu}\right)\left(g_{\xi}^{\nu}\right)^{\prime} d x},
$$

and the initial data $X^{\nu}\left(T_{0}\right)=X_{0}^{\nu}$ is chosen so that

$$
\int_{\mathbb{R}}\left(u^{\nu}-\psi_{X_{0}^{\nu}}^{\nu}\right)\left(x, T_{0}\right) d x=0 .
$$

Proposition 2.5. Under the assumptions of Theorem 1.1, there exists a unique $X_{0}^{\nu} \in$ $\mathbb{R}$ such that (2.17) holds, and the problem (2.15) admits a unique $C^{\infty}$ solution $X^{\nu}(t)$ with

$$
\left|X^{\nu}(t)-s t-X_{\infty}^{\nu}\right| \leqslant C e^{-\alpha t}, \quad t \geqslant T_{0},
$$

where $X_{\infty}^{\nu}$ is the constant defined in Theorem 1.1 and the constant $C>0$ is independent of time $t$.

Remark 2.6. The choice of $X^{\nu}(t)$ can make the source term $h_{X^{\nu}}^{\nu}(x, t)$ and its antiderivative variable $\int_{-\infty}^{x} h_{X^{\nu}}^{\nu}(y, t) d y$ decay exponentially fast both in space and in time (see Proposition 3.5 for details), which plays an important role in the proof of Theorem 1.1.

With the shift curve $X^{\nu}(t)$ so determined, we have the following results. 
Proposition 2.7. Under the assumptions of Theorem 1.1, there exist constants $C>$ 0 and $0<\mu \leqslant \alpha$, independent of time $t$, such that the unique bounded solution $u^{\nu}$ to (1.1), (1.2) satisfies

$$
\sup _{x \in \mathbb{R}}\left|u^{\nu}(x, t)-\psi_{X^{\nu}(t)}^{\nu}(x, t)\right| \leqslant C e^{-\mu t}, \quad t \geqslant T_{0} .
$$

Proposition 2.8. Assume that $f(u)=u^{2} / 2$ in (1.1), and the initial data satisfies

$$
u_{0}(x)=\phi^{\nu}(x)+w_{0}(x),
$$

where $w_{0}(x)$ is periodic with period $p>0$ and zero average. Then at each time $t_{k}=k p /\left(\bar{u}_{l}-\bar{u}_{r}\right), k=0,1,2, \cdots$, the solution $u^{\nu}$ to (1.1), (1.2) satisfies that

$$
u^{\nu}\left(x, t_{k}\right)=\psi_{s t_{k}}^{\nu}\left(x, t_{k}\right), \quad x \in \mathbb{R} .
$$

And the shift function $X^{\nu}$ in Proposition 2.5 satisfies that for $t_{k} \geqslant T_{0}$,

$$
X^{\nu}\left(t_{k}\right)=s t_{k} .
$$

Remark 2.9. It can follow from (2.21) and (2.22) that for $t_{k} \geqslant T_{0}, u^{\nu}\left(x, t_{k}\right)=$ $\psi_{X^{\nu}}^{\nu}\left(x, t_{k}\right)$, which is compatible with (2.19). And it also implies that the ansatz $\psi_{X^{\nu}}^{\nu}$ is a suitable choice to approach the actual solution $u^{\nu}$.

This paper proceeds as follows: We first prove Theorems 1.1, 1.2 and 1.3 for viscous shock profiles in Section 3. More precisely, it is shown that Theorem 1.1 follows from Propositions 2.5 and 2.7 easily. Proposition 2.5 for the shift function $X^{\nu}(t)$ is proved in Section 3.1. Proposition 2.7 is proved in Section 3.2, which is independent of Proposition 2.5. In Section 3.3, we prove the result (1) in Theorem 1.2 and Proposition 2.8 for the Burgers' equation. The proof of Theorem 1.2 is completed in Section 3.4, where a strictly convex flux $f$ is constructed such that $X_{\infty, 2}^{\nu} \neq 0$. In Section 3.5, we prove Theorem 1.3 for the vanishing viscosity limit for $X_{\infty, 2}^{\nu}$. At last, Theorem 1.4 for rarefaction waves is proved in Section 4.

\section{Stability of SHOCK PROFILES}

Theorem 1.1 can follow from Lemma 2.1, Propositions 2.5 and 2.7. In fact, it holds that

$$
\begin{aligned}
\left|u^{\nu}(x, t)-\phi^{\nu}\left(x-s t-X_{\infty}^{\nu}\right)\right| \leqslant & \left|u^{\nu}(x, t)-\psi_{X^{\nu}}^{\nu}(x, t)\right|+\left|\psi_{X^{\nu}}^{\nu}(x, t)-\phi^{\nu}\left(x-X^{\nu}(t)\right)\right| \\
& +\left|\phi^{\nu}\left(x-X^{\nu}(t)\right)-\phi^{\nu}\left(x-s t-X_{\infty}^{\nu}\right)\right| \\
\leqslant & C e^{-\mu t}+\left|u_{l}^{\nu}(x, t)-\bar{u}_{l}\right|+\left|u_{r}^{\nu}(x, t)-\bar{u}_{r}\right|+C e^{-\alpha t} \\
\leqslant & C e^{-\mu t}
\end{aligned}
$$

which proves (1.9). Thus, it remains to prove Propositions 2.5 and 2.7 to finish the proof of Theorem 1.1.

\subsection{Shift function.}




\subsubsection{Existence and uniqueness of the shift function.}

For any $\xi \in \mathbb{R}$,

$$
\int_{\mathbb{R}}\left(u^{\nu}-\psi_{\xi}^{\nu}\right)\left(x, T_{0}\right) d x=\int_{\mathbb{R}}\left(u^{\nu}-u_{l}^{\nu} g_{\xi}^{\nu}-u_{r}^{\nu}\left(1-g_{\xi}^{\nu}\right)\right)\left(x, T_{0}\right) d x .
$$

As $\xi \rightarrow-\infty$ (resp., $+\infty), g_{\xi}^{\nu}(x)=g^{\nu}(x-\xi) \rightarrow 0$ (resp., 1), then due to (2.2) and (2.13), one has that $\int_{\mathbb{R}}\left(u^{\nu}-\psi_{\xi}^{\nu}\right)\left(x, T_{0}\right) d x \rightarrow+\infty$ (resp., $\left.-\infty\right)$ as $\xi \rightarrow-\infty$ (resp., $\left.+\infty\right)$. Thus, there exists an $X_{0}^{\nu} \in \mathbb{R}$ such that (2.17) holds. And the uniqueness follows from (2.13) and the strict monotonicity of $g^{\nu}$.

Now we will prove the existence and uniqueness of $X^{\nu}(t)$ solving the problem (2.15).

Lemma 3.1. The problem (2.15) has a unique $C^{\infty}$ solution $X^{\nu}(t):\left[T_{0},+\infty\right) \rightarrow \mathbb{R}$, satisfying

$$
\left|\left(X^{\nu}\right)^{\prime}(t)-s\right| \leqslant C e^{-\alpha t}, \quad t \geqslant T_{0},
$$

where $C>0$ is independent of time $t$.

Proof. By Lemma 2.4 and (2.13), the denominator of $F^{\nu}(\xi, t)$ satisfies that

$$
\begin{aligned}
\int_{\mathbb{R}}\left(u_{l}^{\nu}-u_{r}^{\nu}\right)(x, t)\left(g_{\xi}^{\nu}\right)^{\prime}(x) d x & \leqslant \frac{\bar{u}_{l}-\bar{u}_{r}}{2} \int_{\mathbb{R}}\left(g_{\xi}^{\nu}\right)^{\prime}(x) d x \\
& =-\frac{\bar{u}_{l}-\bar{u}_{r}}{2}<0 .
\end{aligned}
$$

Since for any $k \geqslant 1,\left(g^{\nu}\right)^{(k)}$ is integrable and $u_{l}^{\nu}, u_{r}^{\nu}$ are bounded and smooth, thus $F^{\nu}$ is smooth and all the derivatives are bounded. Then the existence and uniqueness of $X^{\nu}$ can follow from the Cauchy-Lipschitz theorem.

Now we prove (3.1). By Lemmas 2.1 and 2.4, one can get that

$$
\begin{aligned}
\int_{-\infty}^{+\infty}\left(u_{l}^{\nu}-u_{r}^{\nu}\right)\left(g_{X^{\nu}}^{\nu}\right)^{\prime \prime} d x & =\int_{-\infty}^{+\infty}\left(u_{l}^{\nu}-\bar{u}_{l}-u_{r}+\bar{u}_{r}\right)\left(g_{X^{\nu}}^{\nu}\right)^{\prime \prime} d x=O\left(e^{-\alpha t}\right), \\
\int_{-\infty}^{+\infty}\left(f\left(u_{l}^{\nu}\right)-f\left(u_{r}^{\nu}\right)\right)\left(g_{X^{\nu}}^{\nu}\right)^{\prime} d x & =-f\left(\bar{u}_{l}\right)+f\left(\bar{u}_{r}\right)+O\left(e^{-\alpha t}\right), \\
\int_{-\infty}^{+\infty}\left(u_{l}^{\nu}-u_{r}^{\nu}\right)\left(g_{X^{\nu}}^{\nu}\right)^{\prime} d x & =-\bar{u}_{l}+\bar{u}_{r}+O\left(e^{-\alpha t}\right) .
\end{aligned}
$$

Therefore, (3.1) holds true.

\subsubsection{The limit of the shift function as $t \rightarrow+\infty$.}

In order to compute $\lim _{t \rightarrow+\infty} X^{\nu}(t)-s t$ in terms of the initial data $u_{0}(x)$, the information of the solution $u^{\nu}(x, t)$ for $t \in\left[0, T_{0}\right]$ should be used. However, when $t \in\left[0, T_{0}\right]$, it may fail to find a unique $\xi$ such that $\int_{\mathbb{R}}\left(u^{\nu}-\psi_{\xi}^{\nu}\right)(x, t) d x=0$ or fail to ensure the denominator of $F^{\nu}, \int_{\mathbb{R}}\left(u_{l}^{\nu}-u_{r}^{\nu}\right)(x, t)\left(g_{\xi}^{\nu}\right)^{\prime}(x) d x$, is non-zero. Therefore, we need the following modifications to extend the definition of $X^{\nu}(t)$ on $\left[0, T_{0}\right]$.

For the bounded periodic solutions $u_{l}^{\nu}$ and $u_{r}^{\nu}$, one can first choose a large number $M>0$ such that for all $t \geqslant 0$,

$$
\begin{aligned}
\int_{\mathbb{R}}\left(u_{l}^{\nu}-u_{r}^{\nu}\right)(x, t)\left(g_{\xi}^{\nu}\right)^{\prime}(x) d x-M & \leqslant C \int_{\mathbb{R}}\left|\left(g_{\xi}^{\nu}\right)^{\prime}(x)\right| d x-M \\
& =C-M<0 .
\end{aligned}
$$


Thanks to (3.2), there exists a unique solution $\hat{X}^{\nu}(t) \in C^{1}\left[0, T_{0}\right]$ to the problem

$$
\left\{\begin{array}{l}
\left(\hat{X}^{\nu}\right)^{\prime}(t)=\hat{F}^{\nu}\left(\hat{X}^{\nu}(t), t\right), \quad t \leqslant T_{0}, \\
\hat{X}^{\nu}\left(T_{0}\right)=X_{0}^{\nu}
\end{array}\right.
$$

where

$$
\hat{F}^{\nu}(\xi, t):=\frac{\int_{\mathbb{R}}\left[\nu\left(u_{l}^{\nu}-u_{r}^{\nu}\right)\left(g_{\xi}^{\nu}\right)^{\prime \prime}+\left(f\left(u_{l}^{\nu}\right)-f\left(u_{r}^{\nu}\right)\right)\left(g_{\xi}^{\nu}\right)^{\prime}\right] d x}{\int_{\mathbb{R}}\left(u_{l}^{\nu}-u_{r}^{\nu}\right)\left(g_{\xi}^{\nu}\right)^{\prime} d x-M} .
$$

The proof of existence and uniqueness of $\hat{X}^{\nu}$ is similar to that in Lemma 3.1. Now we claim that

$$
M\left(X_{0}^{\nu}-\hat{X}_{0}^{\nu}\right)+\int_{\mathbb{R}}\left(u_{0}(x)-\psi_{\hat{X}_{0}^{\nu}}^{\nu}(x, 0)\right) d x=0,
$$

where $\hat{X}_{0}^{\nu}:=\hat{X}^{\nu}(0)$.

Indeed, for any $N>0$, one can choose a cut-off function $\varphi_{N}(x) \in C_{0}^{\infty}(\mathbb{R})$ satisfying $\varphi_{N}(x)=1$, if $|x|<N$, and $\varphi_{N}(x)=0$, if $|x|>N+1$. Then by multiplying $\varphi_{N}(x)$ on each side of (2.14) and integrating by parts, one can get that for any $t>0$,

$$
\begin{aligned}
& \int_{\mathbb{R}}\left(u^{\nu}-\psi_{\hat{X}^{\nu}}^{\nu}\right)(x, t) \varphi_{N}(x) d x \\
= & \int_{\mathbb{R}}\left(u^{\nu}-\psi_{\hat{X}^{\nu}}^{\nu}\right)(x, 0) \varphi_{N}(x) d x+\nu \int_{0}^{t} \int_{\mathbb{R}}\left(u^{\nu}-\psi_{\hat{X}^{\nu}}^{\nu}\right) \varphi_{N}^{\prime \prime} d x d \tau \\
& +\int_{0}^{t} \int_{\mathbb{R}}\left(f\left(u^{\nu}\right)-f\left(\psi_{\hat{X}^{\nu}}^{\nu}\right)\right) \varphi_{N}^{\prime} d x d \tau-\int_{0}^{t} \int_{\mathbb{R}} h_{\hat{X}^{\nu}}^{\nu} \varphi_{N} d x d \tau,
\end{aligned}
$$

Thus, by applying Lemmas 2.4 and 3.2 , one can take limit $N \rightarrow+\infty$ and use the dominated convergence theorem in (3.6) to get that

$$
\int_{\mathbb{R}}\left(u^{\nu}-\psi_{\hat{X}^{\nu}}^{\nu}\right)(x, t) d x=\int_{\mathbb{R}}\left(u_{0}(x)-\psi_{\hat{X}_{0}^{\nu}}^{\nu}(x, 0)\right) d x-\int_{0}^{t} \int_{\mathbb{R}} h_{\hat{X}^{\nu}}^{\nu}(x, \tau) d x d \tau .
$$

It follows from (2.11) and (3.3) that

$$
-\int_{\mathbb{R}} h_{\hat{X}^{\nu}}^{\nu}(x, t) d x=M \frac{d}{d t} \hat{X}^{\nu}(t) .
$$

This, together with (2.17) and (3.7), yields (3.5).

Now, we define

$$
\tilde{X}^{\nu}(t):= \begin{cases}X^{\nu}(t), & t>T_{0}, \\ \hat{X}^{\nu}(t), & 0 \leqslant t \leqslant T_{0}\end{cases}
$$

then $\tilde{X}^{\nu}$ is a Lipschitz continuous curve on $[0,+\infty)$. For $y \in(0,1), N \in \mathbb{N}^{*}$ and $t>T_{0}$, we define the domain

$$
\Omega_{y}^{N}:=\left\{(x, \tau): \tilde{X}^{\nu}(\tau)+(-N+y) p_{l} \leqslant x \leqslant \tilde{X}^{\nu}(\tau)+(N+y) p_{r}, 0 \leqslant \tau \leqslant t\right\} ;
$$

see Figure 1.

It follows from the equations of $u_{l}^{\nu}$ and $u_{r}^{\nu}$ that

$$
\iint_{\Omega_{y}^{N}}\left\{\left(\partial_{t} u_{l}^{\nu}+\partial_{x} f\left(u_{l}^{\nu}\right)-\nu \partial_{x}^{2} u_{l}^{\nu}\right) g_{\tilde{X}^{\nu}}^{\nu}+\left(\partial_{t} u_{r}^{\nu}+\partial_{x} f\left(u_{r}^{\nu}\right)-\nu \partial_{x}^{2} u_{r}^{\nu}\right)\left(1-g_{\tilde{X}^{\nu}}^{\nu}\right)\right\} d x d \tau=0 .
$$




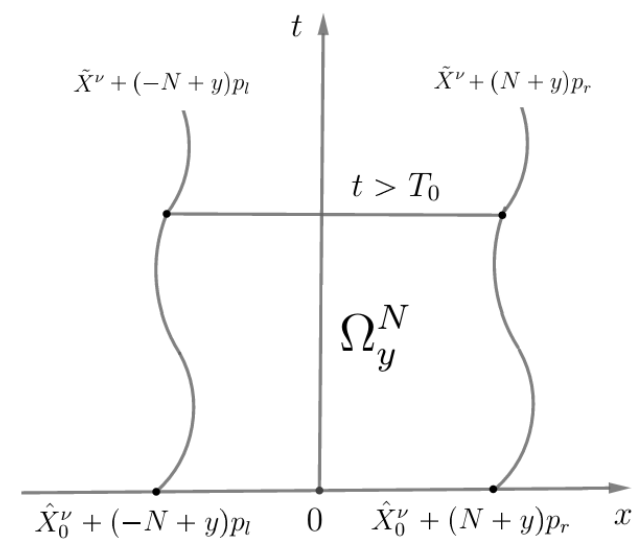

Figure 1.

Then integration by parts yields that

$$
\begin{aligned}
& \iint_{\Omega_{y}^{N}}\left\{-\left(\tilde{X}^{\nu}\right)^{\prime}(t)\left(u_{l}^{\nu}-u_{r}^{\nu}\right)+\left(f\left(u_{l}^{\nu}\right)-\nu \partial_{x} u_{l}^{\nu}\right)-\left(f\left(u_{r}^{\nu}\right)-\nu \partial_{x} u_{r}^{\nu}\right)\right\}\left(g_{\tilde{X}^{\nu}}^{\nu}\right)^{\prime} d x d \tau \\
= & A^{N}(y, t)-A^{N}(y, 0)-B_{l}^{N}(y, t)+B_{r}^{N}(y, t),
\end{aligned}
$$

where

$$
\begin{aligned}
& A^{N}(y, t):= \int_{\tilde{X}^{\nu}(t)+(-N+y) p_{l}}^{\tilde{X}^{\nu}(t)+(N+y) p_{r}}\left[u_{l}^{\nu}(x, t) g_{\tilde{X}^{\nu}}^{\nu}(x)+u_{r}^{\nu}(x, t)\left(1-g_{\tilde{X}^{\nu}}^{\nu}(x)\right)\right] d x, \\
& A^{N}(y, 0):= \int_{\hat{X}_{0}^{\nu}+(-N+y) p_{l}}^{\hat{X}_{0}^{\nu}+(N+y) p_{r}}\left[u_{l}^{\nu}(x, 0) g_{\hat{X}_{0}^{\nu}}^{\nu}(x)+u_{r}^{\nu}(x, 0)\left(1-g_{\hat{X}_{0}^{\nu}}^{\nu}(x)\right)\right] d x, \\
& B_{l}^{N}(y, t):= \int_{0}^{t}\left\{\left(f\left(u_{l}^{\nu}\right)-\nu \partial_{x} u_{l}^{\nu}\right) g_{\tilde{X}^{\nu}}^{\nu}+\left(f\left(u_{r}^{\nu}\right)-\nu \partial_{x} u_{r}^{\nu}\right)\left(1-g_{\tilde{X}^{\nu}}^{\nu}\right)\right. \\
&\left.-\left(\tilde{X}^{\nu}\right)^{\prime}(\tau)\left[u_{l}^{\nu} g_{\tilde{X}^{\nu}}^{\nu}+u_{r}^{\nu}\left(1-g_{\tilde{X}^{\nu}}^{\nu}\right)\right]\right\}\left(\tilde{X}^{\nu}(\tau)+(-N+y) p_{l}, \tau\right) d \tau, \\
& B_{r}^{N}(y, t):=\int_{0}^{t}\left\{\left(f\left(u_{l}^{\nu}\right)-\nu \partial_{x} u_{l}^{\nu}\right) g_{\tilde{X}^{\nu}}^{\nu}+\left(f\left(u_{r}^{\nu}\right)-\nu \partial_{x} u_{r}^{\nu}\right)\left(1-g_{\tilde{X}^{\nu}}^{\nu}\right)\right. \\
&\left.-\left(\tilde{X}^{\nu}\right)^{\prime}(\tau)\left[u_{l}^{\nu} g_{\tilde{X}^{\nu}}^{\nu}+u_{r}^{\nu}\left(1-g_{\tilde{X}^{\nu}}^{\nu}\right)\right]\right\}\left(\tilde{X}^{\nu}(\tau)+(N+y) p_{r}, \tau\right) d \tau .
\end{aligned}
$$

It follows from (2.15) and (3.3) that the left hand side of (3.9)

$$
\begin{gathered}
\iint_{\Omega_{y}^{N}} \cdots d x d \tau=\iint_{\Omega_{y}^{N} \cap\left\{0<\tau<T_{0}\right\}} \cdots d x d \tau+\iint_{\Omega_{y}^{N} \cap\left\{T_{0}<\tau<t\right\}} \cdots d x d \tau \\
\rightarrow \quad-M \int_{0}^{T_{0}}\left(\hat{X}^{\nu}\right)^{\prime}(t) d t+0=-M\left(X_{0}^{\nu}-\hat{X}_{0}^{\nu}\right) \quad \text { as } N \rightarrow+\infty .
\end{gathered}
$$

Thus it remains to evaluate the right hand side of (3.9) as $N \rightarrow+\infty$.

(i) The integrals on $\{\tau=0\}$ and $\{\tau=t\}$.

Set

$$
w_{l}^{\nu}(x, t):=u_{l}^{\nu}(x, t)-\bar{u}_{l}, \quad w_{r}^{\nu}(x, t):=u_{r}^{\nu}(x, t)-\bar{u}_{r} .
$$


Then it follows from Lemma 2.1 that $\left\|w_{l}^{\nu}\right\|_{L^{\infty}}+\left\|w_{r}^{\nu}\right\|_{L^{\infty}} \leqslant C e^{-\alpha t}$. Since $w_{0 l}$ and $w_{0 r}$ are both of zero average, one has that

$$
\begin{aligned}
& J^{N}(y, t)=: A^{N}(y, t)-A^{N}(y, 0) \\
= & \int_{(-N+y) p_{l}}^{(N+y) p_{r}}\left[w_{l}^{\nu}\left(x+\tilde{X}^{\nu}(t), t\right) g^{\nu}(x)+w_{r}^{\nu}\left(x+\tilde{X}^{\nu}(t), t\right)\left(1-g^{\nu}(x)\right)\right] d x \\
& -\int_{\hat{X}_{0}^{\nu}+(-N+y) p_{l}}^{\hat{X}_{0}^{\nu}+(N+y) p_{r}}\left[w_{0 l}(x) g^{\nu}\left(x-\hat{X}_{0}^{\nu}\right)+w_{0 r}(x)\left(1-g^{\nu}\left(x-\hat{X}_{0}^{\nu}\right)\right)\right] d x \\
= & O\left(e^{-\alpha t}\right)+\int_{\hat{X}_{0}^{\nu}+(-N+y) p_{l}}^{\hat{X}_{0}^{\nu}+y p_{l}}\left(w_{0 l}-w_{0 r}\right)(x)\left(1-g^{\nu}\left(x-\hat{X}_{0}^{\nu}\right)\right) d x \\
& -\int_{\hat{X}_{0}^{\nu}+y p_{l}}^{\hat{X}_{0}^{\nu}+y p_{r}}\left[w_{0 l}(x) g^{\nu}\left(x-\hat{X}_{0}^{\nu}\right)+w_{0 r}(x)\left(1-g^{\nu}\left(x-\hat{X}_{0}^{\nu}\right)\right)\right] d x \\
& -\int_{\hat{X}_{0}^{\nu}+y p_{r}}^{\hat{X}_{0}^{\nu}+(N+y) p_{r}}\left(w_{0 l}-w_{0 r}\right)(x) g^{\nu}\left(x-\hat{X}_{0}^{\nu}\right) d x .
\end{aligned}
$$

Then one can get that

$$
\begin{aligned}
J(y, t):= & \lim _{N \rightarrow \infty} J^{N}(y, t) \\
= & O\left(e^{-\alpha t}\right)+\int_{-\infty}^{\hat{X}_{0}^{\nu}+y p_{l}}\left(w_{0 l}-w_{0 r}\right)(x)\left(1-g^{\nu}\left(x-\hat{X}_{0}^{\nu}\right)\right) d x \\
& -\int_{\hat{X}_{0}^{\nu}+y p_{l}}^{\hat{X}_{0}^{\nu}+y p_{r}}\left[w_{0 l}(x) g^{\nu}\left(x-\hat{X}_{0}^{\nu}\right)+w_{0 r}(x)\left(1-g^{\nu}\left(x-\hat{X}_{0}^{\nu}\right)\right)\right] d x \\
& -\int_{\hat{X}_{0}^{\nu}+y p_{r}}^{+\infty}\left(w_{0 l}-w_{0 r}\right)(x) g^{\nu}\left(x-\hat{X}_{0}^{\nu}\right) d x .
\end{aligned}
$$

By (3.5), it holds that

$$
\begin{aligned}
& M\left(X_{0}^{\nu}-\hat{X}_{0}^{\nu}\right)=-\int_{\mathbb{R}}\left[u_{0}(x)-\phi^{\nu}\left(x-\hat{X}_{0}^{\nu}\right)-w_{0 l}(x) g^{\nu}\left(x-\hat{X}_{0}^{\nu}\right)\right. \\
& \left.-w_{0 r}(x)\left(1-g^{\nu}\left(x-\hat{X}_{0}^{\nu}\right)\right)\right] d x \\
& =-\int_{-\infty}^{0}\left(u_{0}-\phi^{\nu}-w_{0 l}\right)(x) d x-\int_{0}^{+\infty}\left(u_{0}-\phi^{\nu}-w_{0 r}\right)(x) d x \\
& +\left(\bar{u}_{l}-\bar{u}_{r}\right) \hat{X}_{0}^{\nu}-\int_{-\infty}^{0}\left(w_{0 l}-w_{0 r}\right)(x)\left(1-g^{\nu}\left(x-\hat{X}_{0}^{\nu}\right)\right) d x \\
& +\int_{0}^{+\infty}\left(w_{0 l}-w_{0 r}\right)(x) g^{\nu}\left(x-\hat{X}_{0}^{\nu}\right) d x
\end{aligned}
$$

This, together with (3.13), yields that

$$
\begin{aligned}
J(y, t)= & O\left(e^{-\alpha t}\right)-M\left(X_{0}^{\nu}-\hat{X}_{0}^{\nu}\right)+\left(\bar{u}_{l}-\bar{u}_{r}\right) \hat{X}_{0}^{\nu} \\
& -\int_{-\infty}^{0}\left(u_{0}-\phi^{\nu}-w_{0 l}\right)(x) d x-\int_{0}^{+\infty}\left(u_{0}-\phi^{\nu}-w_{0 r}\right)(x) d x \\
& +\int_{0}^{\hat{X}_{0}^{\nu}+y p_{l}} w_{0 l}(x) d x-\int_{0}^{\hat{X}_{0}^{\nu}+y p_{r}} w_{0 r}(x) d x .
\end{aligned}
$$


Note that for $i=l, r$, since $w_{0 i}$ has zero average, $\int_{0}^{y} w_{0 i}(x) d x$ is periodic with respective to $y$ with period $p_{i}$. Therefore,

$$
\begin{aligned}
\int_{0}^{1} \int_{0}^{\hat{X}_{0}^{\nu}+y p_{i}} w_{0 i}(x) d x d y & =\frac{1}{p_{i}} \int_{0}^{p_{i}} \int_{0}^{\hat{X}_{0}^{\nu}+y} w_{0 i}(x) d x d y \\
& =\frac{1}{p_{i}} \int_{0}^{p_{i}} \int_{0}^{y} w_{0 i}(x) d x d y .
\end{aligned}
$$

Thus one can get that

$$
\begin{aligned}
\int_{0}^{1} J(y, t) d y= & O\left(e^{-\alpha t}\right)-M\left(X_{0}^{\nu}-\hat{X}_{0}^{\nu}\right)+\left(\bar{u}_{l}-\bar{u}_{r}\right) \hat{X}_{0}^{\nu} \\
& -\int_{-\infty}^{0}\left(u_{0}-\phi^{\nu}-w_{0 l}\right)(x) d x-\int_{0}^{+\infty}\left(u_{0}-\phi^{\nu}-w_{0 r}\right)(x) d x \\
& +\frac{1}{p_{l}} \int_{0}^{p_{l}} \int_{0}^{y} w_{0 l}(x) d x d y-\frac{1}{p_{r}} \int_{0}^{p_{r}} \int_{0}^{y} w_{0 r}(x) d x d y .
\end{aligned}
$$

(ii) The integrals on two sides.

Since $u_{l}^{\nu}$ is periodic, it holds that

$$
\begin{aligned}
B_{l}^{N}(y, t)=\int_{0}^{t}\{ & \left(f\left(u_{l}^{\nu}\right)-\nu \partial_{x} u_{l}^{\nu}\right)\left(\tilde{X}^{\nu}(\tau)+y p_{l}, \tau\right) g^{\nu}\left((-N+y) p_{l}\right) \\
& -\left(\tilde{X}^{\nu}\right)^{\prime}(\tau) u_{l}^{\nu}\left(\tilde{X}^{\nu}(\tau)+y p_{l}, \tau\right) \\
& \left.+[\cdots]\left(1-g_{\tilde{X}^{\nu}}^{\nu}\right)\left(\tilde{X}^{\nu}(\tau)+(-N+y) p_{l}, \tau\right)\right\} d \tau
\end{aligned}
$$

where $[\cdots]$ denotes the remaining terms which are bounded. Then taking the limit $N \rightarrow+\infty$ in (3.16) and using Lemma 2.4, one can get that

$$
\begin{aligned}
\lim _{N \rightarrow+\infty} \int_{0}^{1} B_{l}^{N}(y, t) d y & =\int_{0}^{t} \frac{1}{p_{l}} \int_{0}^{p_{l}} f\left(u_{l}^{\nu}\right)\left(\tilde{X}^{\nu}+x, \tau\right) d x d \tau-\int_{0}^{t}\left(\tilde{X}^{\nu}\right)^{\prime}(\tau) \bar{u}_{l} d \tau \\
& =\int_{0}^{t} \frac{1}{p_{l}} \int_{0}^{p_{l}} f\left(u_{l}^{\nu}\right)(x, \tau) d x d \tau-\bar{u}_{l}\left(\tilde{X}^{\nu}(t)-\hat{X}_{0}^{\nu}\right) .
\end{aligned}
$$

Similarly, it holds that

$$
\lim _{N \rightarrow+\infty} \int_{0}^{1} B_{r}^{N}(y, t) d y=\int_{0}^{t} \frac{1}{p_{r}} \int_{0}^{p_{r}} f\left(u_{r}^{\nu}\right)(x, \tau) d x d \tau-\bar{u}_{r}\left(\tilde{X}^{\nu}(t)-\hat{X}_{0}^{\nu}\right) .
$$

Now, with the calculations in (i) and (ii), one can integrate the equation (3.9) with respect to $y$ over $(0,1)$, and then let $N \rightarrow+\infty$, to get that for any $t>T_{0}$,

$$
\begin{aligned}
& \int_{0}^{1} J(y, t) d y+\left(\bar{u}_{l}-\bar{u}_{r}\right)\left(\tilde{X}^{\nu}(t)-\hat{X}_{0}^{\nu}\right) \\
= & \int_{0}^{t}\left[\frac{1}{p_{l}} \int_{0}^{p_{l}} f\left(u_{l}^{\nu}\right)(x, \tau) d x-\frac{1}{p_{r}} \int_{0}^{p_{r}} f\left(u_{r}^{\nu}\right)(x, \tau) d x\right] d \tau-M\left(X_{0}^{\nu}-\hat{X}_{0}^{\nu}\right) .
\end{aligned}
$$

Note also that for $i=l, r$,

$$
\int_{0}^{t} \frac{1}{p_{i}} \int_{0}^{p_{i}} f\left(u_{i}^{\nu}\right) d y d \tau=\int_{0}^{t} \frac{1}{p_{i}} \int_{0}^{p_{i}}\left(f\left(u_{i}^{\nu}\right)-f\left(\bar{u}_{i}\right)\right) d y d \tau+f\left(\bar{u}_{i}\right) t
$$




$$
=\int_{0}^{+\infty} \frac{1}{p_{i}} \int_{0}^{p_{i}}\left(f\left(u_{i}^{\nu}\right)-f\left(\bar{u}_{i}\right)\right) d y d \tau+O\left(e^{-\alpha t}\right)+f\left(\bar{u}_{i}\right) t
$$

where Lemma 2.1 is used. Then it can follow from (3.15) and (3.17) that for $t>T_{0}$,

$$
X^{\nu}(t)-s t=\tilde{X}^{\nu}(t)-s t=O\left(e^{-\alpha t}\right)+X_{\infty}^{\nu}
$$

where $X_{\infty}^{\nu}$ is defined in Theorem 1.1. The proof of Proposition 2.5 is finished.

\subsection{Decay to ansatz.}

In this section, we will prove Proposition 2.7. First, $u^{\nu}(x, t)$ will be proved to be close to $u_{l}^{\nu}(x, t)$ (resp., $\left.u_{r}^{\nu}(x, t)\right)$ in the region $x<s t-N_{\varepsilon}\left(\right.$ resp. $x>s t-N_{\varepsilon}$ ) with large enough $t$ and $N_{\varepsilon}$ (independent of time); see Proposition 3.4. Then motivated by [16], the equation of the anti-derivative variable of $u^{\nu}-\psi_{X^{\nu}}^{\nu}$ is studied and the comparison principle is applied to prove Proposition 2.7.

\subsubsection{Time-independent estimates.}

The following result follows directly from Lemma 2.3.

Lemma 3.2. Under the assumptions of Theorem 1.1, there holds that

$$
\left|u^{\nu}(x, t)-\psi_{\xi}^{\nu}(x, t)\right| \leqslant C(t) e^{\beta|\xi|} e^{-\beta|x-\xi|}, \quad \xi, x \in \mathbb{R}, t \geqslant 0,
$$

where $C(t)>0$ is bounded on any compact subset of $[0,+\infty)$.

Then by Lemmas 2.3 and 3.2, for any $x \in \mathbb{R}, t \geqslant 0$, one can define the anti-derivative variables:

$$
\begin{aligned}
U_{l}^{\nu}(x, t) & :=\int_{-\infty}^{x}\left(u^{\nu}-u_{l}^{\nu}\right)(y, t) d y, \\
U_{r}^{\nu}(x, t) & :=\int_{x}^{+\infty}\left(u^{\nu}-u_{r}^{\nu}\right)(y, t) d y, \\
\check{U}_{\xi}^{\nu}(x, t): & =\int_{-\infty}^{x}\left(u^{\nu}-\psi_{\xi}^{\nu}\right)(y, t) d y, \\
\hat{U}_{\xi}^{\nu}(x, t) & :=\int_{x}^{+\infty}\left(u^{\nu}-\psi_{\xi}^{\nu}\right)(y, t) d y .
\end{aligned}
$$

Lemma 3.3. For any $C^{\infty}$ curve $\xi(t):[0,+\infty) \rightarrow \mathbb{R}$ with bounded derivatives, the functions $U_{l}^{\nu}, U_{r}^{\nu}, \check{U}_{\xi}^{\nu}$ and $\hat{U}_{\xi}^{\nu}$ defined above satisfy the following equations:

$$
\begin{aligned}
& \partial_{t} U_{l}^{\nu}-\nu \partial_{x}^{2} U_{l}^{\nu}=f\left(u_{l}^{\nu}\right)-f\left(u^{\nu}\right), \quad x \in \mathbb{R}, t>1, \\
& \partial_{t} U_{r}^{\nu}-\nu \partial_{x}^{2} U_{r}^{\nu}=f\left(u^{\nu}\right)-f\left(u_{r}^{\nu}\right), \quad x \in \mathbb{R}, t>1, \\
& \partial_{t} \check{U}_{\xi}^{\nu}-\nu \partial_{x}^{2} \check{U}_{\xi}^{\nu}=f\left(\psi_{\xi}^{\nu}\right)-f\left(u^{\nu}\right)-\int_{-\infty}^{x} h_{\xi}^{\nu}(y, t) d y, \quad x \in \mathbb{R}, t>1, \\
& \partial_{t} \hat{U}_{\xi}^{\nu}-\nu \partial_{x}^{2} \hat{U}_{\xi}^{\nu}=f\left(u^{\nu}\right)-f\left(\psi_{\xi}^{\nu}\right)-\int_{x}^{+\infty} h_{\xi}^{\nu}(y, t) d y, \quad x \in \mathbb{R}, t>1,
\end{aligned}
$$

where the derivatives appearing in these equations are all continuous in $\mathbb{R} \times[1,+\infty)$. Proof. Here we prove only (3.23) and (3.25), since the proofs of the other two are similar. 
(1) To prove (3.23), for any $T>1$, one considers the following problem:

$$
\left\{\begin{array}{l}
\partial_{t} V-\nu \partial_{x}^{2} V=f\left(u_{l}^{\nu}\right)-f\left(u^{\nu}\right), \quad x \in \mathbb{R}, 1<t \leqslant T, \\
V(x, 1)=U_{l}^{\nu}(x, 1) .
\end{array}\right.
$$

It follows from Lemma 3.2 that

$$
\begin{cases}\left|f\left(u_{l}^{\nu}\right)-f\left(u^{\nu}\right)\right| \leqslant C(T) e^{\beta x} & \forall x \in \mathbb{R}, 1<t \leqslant T, \\ \left|U_{l}^{\nu}(x, 1)\right| \leqslant C e^{\beta x} & \forall x \in \mathbb{R} .\end{cases}
$$

Note that

$$
e^{\beta|x|} \leqslant C(T) e^{\frac{1}{4 \nu T}|x|^{2}},
$$

and the initial data $U_{l}^{\nu}(x, 1)$ and the source term $f\left(u_{l}^{\nu}\right)-f\left(u^{\nu}\right)$ are smooth functions, then by the standard parabolic theories (see [6, Chapter 1, Theorem 12]), the function

$$
V(x, t)=\int_{0}^{t-1} K^{\tau}(\cdot) *\left(f\left(u_{l}^{\nu}\right)-f\left(u^{\nu}\right)\right)(\cdot, t-\tau) d \tau+K^{t-1}(\cdot) * U_{l}^{\nu}(\cdot, 1)
$$

solves (3.27) and all the derivatives of $V$ appearing in the equation exist and are continuous in $\mathbb{R} \times[1, T]$, where $K^{t}(x):=(2 \pi \nu t)^{-\frac{1}{2}} e^{-\frac{x^{2}}{4 \nu t}}$ denotes the heat kernel. It then follows from (3.28) and (3.29) that $V(x, t)$ vanishes as $x \rightarrow-\infty$. Then by (3.29) and the equations of $u^{\nu}$ and $u_{l}^{\nu}$, it holds that

$$
\begin{aligned}
\partial_{x} V(x, t)= & \int_{0}^{t-1}\left[\partial_{x} K^{\tau} *\left(f\left(u_{l}^{\nu}\right)-f(u)\right)\right](x, t-\tau) d \tau \\
& +\left[K^{t-1} *\left(u-u_{l}^{\nu}\right)\right](x, 1) \\
= & \left(u-u_{l}^{\nu}\right)(x, t), \quad x \in \mathbb{R}, 1 \leqslant t \leqslant T,
\end{aligned}
$$

which implies that $V(x, t)=U_{l}^{\nu}(x, t)$ for all $x \in \mathbb{R}$ and $1 \leqslant t \leqslant T$. And since $T>1$ is arbitrary, (3.23) holds true.

(2) Now we prove (3.25). By integrating (2.11) with the space variable on $(-\infty, x)$, one can get from Lemmas 2.1 and 2.4 that

$$
\begin{aligned}
\int_{-\infty}^{x} h_{\xi}^{\nu} d y= & \left(f\left(\psi_{\xi}^{\nu}\right)-f\left(u_{l}^{\nu}\right)\right) g_{\xi}^{\nu}+\left(f\left(\psi_{\xi}^{\nu}\right)-f\left(u_{r}^{\nu}\right)\right)\left(1-g_{\xi}^{\nu}\right) \\
& -2 \nu\left(u_{l}^{\nu}-u_{r}^{\nu}\right)\left(g_{\xi}^{\nu}\right)^{\prime}+\int_{-\infty}^{x}\left(f\left(u_{l}^{\nu}\right)-f\left(u_{r}^{\nu}\right)\right)\left(g_{\xi}^{\nu}\right)^{\prime} d y \\
& -\xi^{\prime}(t) \int_{-\infty}^{x}\left(u_{l}^{\nu}-u_{r}^{\nu}\right)\left(g_{\xi}^{\nu}\right)^{\prime} d y+\nu \int_{-\infty}^{x}\left(u_{l}^{\nu}-u_{r}^{\nu}\right)\left(g_{\xi}^{\nu}\right)^{\prime \prime} d y
\end{aligned}
$$

is smooth. And it follows from Lemmas 2.4, 3.2 and $\psi_{\xi}^{\nu}-u_{l}^{\nu}=-\left(u_{l}^{\nu}-u_{r}^{\nu}\right)(1-$ $\left.g_{\xi}^{\nu}\right)$ that for any $T>1$,

$$
\left|\int_{-\infty}^{x} h_{\xi}^{\nu} d y\right| \leqslant C(T)\left[1-g_{\xi}^{\nu}(x)+\left|\left(g_{\xi}^{\nu}\right)^{\prime}(x)\right|+\int_{-\infty}^{x}\left|\left(g_{\xi}^{\nu}\right)^{\prime}\right| d y+\int_{-\infty}^{x}\left|\left(g_{\xi}^{\nu}\right)^{\prime \prime}\right| d y\right] \text {. }
$$

Note that $\int_{-\infty}^{x}\left|\left(g_{\xi}^{\nu}\right)^{\prime}\right| d y=1-g_{\xi}^{\nu}$. And by $\left(g^{\nu}\right)^{\prime \prime}=\frac{1}{\bar{u}_{l}-\bar{u}_{r}}\left(f^{\prime}\left(\phi^{\nu}\right)-s\right)\left(\phi^{\nu}\right)^{\prime}$, there exists a unique point $x_{0} \in \mathbb{R}$ such that $\left(g^{\nu}\right)^{\prime \prime}<0$ for $x<x_{0}$, and $\left(g^{\nu}\right)^{\prime \prime}>0$ for $x>x_{0}$. Then $\int_{-\infty}^{x}\left|\left(g_{\xi}^{\nu}\right)^{\prime \prime}\right| d y=-\left(g_{\xi}^{\nu}\right)^{\prime}$ if $x<x_{0}+\xi$. Hence, it follows from Lemma 2.4 that

$$
\left|\int_{-\infty}^{x} h_{\xi}^{\nu} d y\right| \leqslant C(T) e^{\beta x} \quad \forall x \in \mathbb{R}, 1 \leqslant t \leqslant T .
$$


By Lemma 3.2, one can verify easily that

$$
\left|\check{U}_{\xi(1)}^{\nu}(x, 1)\right| \leqslant C e^{\beta x} \quad \forall x \in \mathbb{R} .
$$

Now we consider the problem:

$$
\begin{cases}\partial_{t} \check{V}-\nu \partial_{x}^{2} \check{V}=\check{H}^{\nu}:=f\left(\psi_{\xi}^{\nu}\right)-f\left(u^{\nu}\right)-\int_{-\infty}^{x} h_{\xi}^{\nu}(y, t) d y, & x \in \mathbb{R}, 1<t \leqslant T, \\ \check{V}(x, 1)=\check{U}_{\xi(1)}^{\nu}(x, 1), & x \in \mathbb{R} .\end{cases}
$$

By Lemma 3.2 and (3.30), $\check{H}^{\nu}$ satisfies that

$$
\left|\check{H}^{\nu}(x, t)\right| \leqslant C(T) e^{\beta x} \quad \forall x \in \mathbb{R}, 1<t \leqslant T .
$$

Then similar to the proof in $(1)$, since the initial data $\check{U}_{\xi(1)}^{\nu}(x, 1)$ is smooth and satisfies (3.31), and the source term $\check{H}^{\nu}$ is smooth and satisfies (3.33), one can obtain that the function

$$
\check{V}(x, t)=\int_{0}^{t-1} K^{\tau}(\cdot) * \check{H}^{\nu}(\cdot, t-\tau) d \tau+K^{t-1}(\cdot) * \check{U}_{\xi(1)}^{\nu}(\cdot, 1)
$$

solves (3.32) and all the derivatives of $\check{V}$ appearing in the equation exist and are continuous in $\mathbb{R} \times[1, T]$. It follows from (3.33) and (3.34) that $\check{V}(x, t)$ vanishes as $x \rightarrow-\infty$. Then (3.34) and the equation of $\psi_{\xi}^{\nu}$ yield

$$
\begin{aligned}
\partial_{t}\left(u^{\nu}-\psi_{\xi}^{\nu}\right)-\nu \partial_{x}^{2}\left(u^{\nu}-\psi_{\xi}^{\nu}\right) & =\partial_{x}\left[f\left(\psi_{\xi}^{\nu}\right)-f\left(u^{\nu}\right)-\int_{-\infty}^{x} h_{\xi}^{\nu}(y, t) d y\right] \\
& =\partial_{x} \check{H}^{\nu}
\end{aligned}
$$

And then

$$
\begin{aligned}
\partial_{x} \check{V}(x, t) & =\int_{0}^{t-1} \partial_{x} K^{\tau}(\cdot) * \check{H}^{\nu}(\cdot, t-\tau) d \tau+K^{t-1}(\cdot) *\left(u^{\nu}-\psi_{\xi}^{\nu}\right)(\cdot, 1) \\
& =\left(u^{\nu}-\psi_{\xi}^{\nu}\right)(x, t) \quad \forall x \in \mathbb{R}, 1<t \leqslant T,
\end{aligned}
$$

which implies that $\check{V}=\check{U}_{\xi}^{\nu}$.

Now we choose a fixed small number $\varepsilon_{0}>0$ such that

$$
f^{\prime}\left(\bar{u}_{l}-2 \varepsilon_{0}\right)-s>0 \text { and } f^{\prime}\left(\bar{u}_{r}+2 \varepsilon_{0}\right)-s<0 .
$$

Then it can follow from Lemma 2.1 that there exists $T_{1}>T_{0}$ large enough such that

$$
u_{l}^{\nu}(x, t)>\bar{u}_{l}-\varepsilon_{0} \text { and } u_{r}^{\nu}(x, t)<\bar{u}_{r}+\varepsilon_{0} \quad \forall x \in \mathbb{R}, t>T_{1},
$$

where $T_{0}$ is the number chosen in (2.13).

For later use, we define

$$
\begin{aligned}
& a(v, w):=\int_{0}^{1} f^{\prime}(w+\rho(v-w)) d \rho \\
& b(v, w):=\int_{0}^{1} f^{\prime \prime}(w+\rho(v-w)) d \rho .
\end{aligned}
$$

Now, we give the results of the time-independent estimates of $u^{\nu}-u_{l}^{\nu}$ and $u^{\nu}-u_{r}^{\nu}$. 
Proposition 3.4. Under the assumptions of Theorem 1.1. There exists $T_{2}>T_{1}$ such that for any $\varepsilon>0$, there exists $N(\varepsilon)>0$, independent of time $t$, such that

$$
\begin{array}{ll}
\left|u^{\nu}(x, t)-u_{l}^{\nu}(x, t)\right| \leqslant \varepsilon, & t>T_{2}, x<s t-N(\varepsilon), \\
\left|u^{\nu}(x, t)-u_{r}^{\nu}(x, t)\right| \leqslant \varepsilon, & t>T_{2}, x>s t+N(\varepsilon) .
\end{array}
$$

Proof. We prove (3.39) only, since the proof of (3.40) is similar. And the proof will be divided into four steps.

Step 1 . We will prove that there exist $T_{2}>T_{1}$, independent of $\varepsilon$, and $N_{1}=N_{1}(\varepsilon)>$ 0 such that

$$
U_{l}^{\nu}(x, t)<\varepsilon, \quad t>T_{2}, x<s t-N_{1},
$$

where $U_{l}^{\nu}$ is defined in (3.19).

By (3.35), one can define a constant

$$
\tilde{\beta}:=f^{\prime}\left(\bar{u}_{l}-2 \varepsilon_{0}\right)-s>0 .
$$

For a constant $M_{1}>1$ to be determined later (see (3.47)), we define the function:

$$
\tilde{u}(x, t):=M_{1} e^{\tilde{\beta}(x-s t)}+u_{l}^{\nu}(x, t), \quad x \in \mathbb{R}, t>0 .
$$

Then by the equation of $u_{l}^{\nu}$, one has that

$$
\partial_{t} \tilde{u}-\nu \partial_{x}^{2} \tilde{u}+\partial_{x} f(\tilde{u})=\tilde{h}:=-\tilde{\beta} s M_{1} e^{\tilde{\beta}(x-s t)}-\nu \tilde{\beta}^{2} M_{1} e^{\tilde{\beta}(x-s t)}+\partial_{x}\left(f(\tilde{u})-f\left(u_{l}^{\nu}\right)\right) .
$$

Since $f$ is strictly convex, (3.36) implies that

$$
f^{\prime}(\tilde{u}) \geqslant f^{\prime}\left(u_{l}^{\nu}\right) \geqslant f^{\prime}\left(\bar{u}_{l}-\varepsilon_{0}\right) \quad \forall x \in \mathbb{R}, t \geqslant T_{1} .
$$

Hence, for the given constant $M_{1}$, if $t \geqslant T_{1}$, the following two inequalities hold.

(1) If $x$ satisfies $M e^{\tilde{\beta}(x-s t)} \geqslant 1$, then it holds that

$$
\begin{aligned}
\partial_{x}\left(f(\tilde{u})-f\left(u_{l}^{\nu}\right)\right) & =\left(f^{\prime}(\tilde{u})-f^{\prime}\left(u_{l}^{\nu}\right)\right)\left(\tilde{\beta} M_{1} e^{\tilde{\beta}(x-s t)}+\partial_{x} u_{l}^{\nu}\right)+f^{\prime}\left(u_{l}^{\nu}\right) \tilde{\beta} M_{1} e^{\tilde{\beta}(x-s t)} \\
& \geqslant\left(f^{\prime}(\tilde{u})-f^{\prime}\left(u_{l}^{\nu}\right)\right)\left(\tilde{\beta}-\left|\partial_{x} u_{l}^{\nu}\right|\right)+f^{\prime}\left(\bar{u}_{l}-\varepsilon_{0}\right) \tilde{\beta} M_{1} e^{\tilde{\beta}(x-s t)} .
\end{aligned}
$$

Then by (3.43), Lemma 2.4 and $\nu \leqslant 1$, one has that

$$
\begin{aligned}
\tilde{h} & \geqslant\left(f^{\prime}(\tilde{u})-f^{\prime}\left(u_{l}^{\nu}\right)\right)\left(\tilde{\beta}-\left|\partial_{x} u_{l}^{\nu}\right|\right)+\tilde{\beta} M_{1} e^{\tilde{\beta}(x-s t)}\left(f^{\prime}\left(\bar{u}_{l}-\varepsilon_{0}\right)-(s+\tilde{\beta})\right) \\
& \geqslant\left(f^{\prime}(\tilde{u})-f^{\prime}\left(u_{l}^{\nu}\right)\right)\left(\tilde{\beta}-C e^{-\alpha t}\right)+\tilde{\beta} M_{1} e^{\tilde{\beta}(x-s t)}\left(f^{\prime}\left(\bar{u}_{l}-\varepsilon_{0}\right)-f^{\prime}\left(\bar{u}_{l}-2 \varepsilon_{0}\right)\right) \\
& \geqslant\left(f^{\prime}(\tilde{u})-f^{\prime}\left(u_{l}^{\nu}\right)\right)\left(\tilde{\beta}-C e^{-\alpha t}\right) .
\end{aligned}
$$

(2) If $x$ satisfies $M_{1} e^{\tilde{\beta}(x-s t)}<1$, one has $0<\tilde{u}-u_{l}^{\nu} \leqslant 1$. Then it holds that

$$
\begin{aligned}
\partial_{x}\left(f(\tilde{u})-f\left(u_{l}^{\nu}\right)\right) & =f^{\prime}(\tilde{u}) \tilde{\beta} M e^{\tilde{\beta}(x-s t)}+\left(f^{\prime}(\tilde{u})-f^{\prime}\left(u_{l}^{\nu}\right)\right) \partial_{x} u_{l}^{\nu} \\
& =f^{\prime}(\tilde{u}) \tilde{\beta} M_{1} e^{\tilde{\beta}(x-s t)}+f^{\prime \prime}(\cdot)\left(\tilde{u}-u_{l}^{\nu}\right) \partial_{x} u_{l}^{\nu} \\
& \geqslant f^{\prime}\left(\bar{u}_{l}-\varepsilon_{0}\right) \tilde{\beta} M_{1} e^{\tilde{\beta}(x-s t)}-C M_{1} e^{\tilde{\beta}(x-s t)}\left|\partial_{x} u_{l}^{\nu}\right| .
\end{aligned}
$$

This yields that

$$
\begin{aligned}
\tilde{h} & \geqslant \tilde{\beta} M_{1} e^{\tilde{\beta}(x-s t)}\left(f^{\prime}\left(\bar{u}_{l}-\varepsilon_{0}\right)-(s+\tilde{\beta})\right)-C M_{1} e^{\tilde{\beta}(x-s t)}\left|\partial_{x} u_{l}^{\nu}\right| \\
& \geqslant M_{1} e^{\tilde{\beta}(x-s t)}\left[\tilde{\beta}\left(f^{\prime}\left(\bar{u}_{l}-\varepsilon_{0}\right)-(s+\tilde{\beta})\right)-C e^{-\alpha t}\right]
\end{aligned}
$$




$$
\begin{aligned}
& \geqslant M_{1} e^{\tilde{\beta}(x-s t)}\left[\tilde{\beta}\left(f^{\prime}\left(\bar{u}_{l}-\varepsilon_{0}\right)-f^{\prime}\left(\bar{u}_{l}-2 \varepsilon_{0}\right)\right)-C e^{-\alpha t}\right] \\
& \geqslant M_{1} e^{\tilde{\beta}(x-s t)}\left(\tilde{\beta} c_{0} \varepsilon_{0}-C e^{-\alpha t}\right),
\end{aligned}
$$

where $c_{0}:=\min f^{\prime \prime}>0$.

As a result of (1) and (2), there exists a constant $T_{2}>T_{1}$, independent of either $M_{1}$ or $\varepsilon$, such that

$$
\tilde{h}=\partial_{t} \tilde{u}-\nu \partial_{x}^{2} \tilde{u}+\partial_{x} f(\tilde{u})>0, \quad x \in \mathbb{R}, t \geqslant T_{2} .
$$

Set $\tilde{U}(x, t):=\int_{-\infty}^{x}\left(u^{\nu}-\tilde{u}\right)(y, t) d y$. Then as for the proof of Lemma 3.3, one can prove that

$$
\partial_{t} \tilde{U}-\nu \partial_{x}^{2} \tilde{U}+\left(f\left(u^{\nu}\right)-f(\tilde{u})\right)=-\int_{-\infty}^{x} \tilde{h}(y, t) d y, \quad x \in \mathbb{R}, t>T_{2},
$$

which implies that

$$
\partial_{t} \tilde{U}-\nu \partial_{x}^{2} \tilde{U}+a\left(u^{\nu}, \tilde{u}\right) \partial_{x} \tilde{U}<0 \quad \forall x \in \mathbb{R}, t>T_{2},
$$

where $a(u, v)$ is defined by (3.37).

By Lemma 3.2, there exists $N_{1}>0$ such that

$$
\left|U_{l}^{\nu}\left(x, T_{2}\right)\right|=\left|\int_{-\infty}^{x}\left(u^{\nu}-u_{l}^{\nu}\right)\left(y, T_{2}\right) d y\right| \leqslant \varepsilon \quad \forall x \leqslant-N_{1} .
$$

For this $N_{1}$, it can also follow from Lemma 3.2 that one can define a constant

$$
B:=\sup _{x \geqslant-N_{1}}\left|\int_{-N_{1}}^{x}\left(u^{\nu}-u_{r}^{\nu}\right)\left(y, T_{2}\right) d y\right|<+\infty .
$$

Thus, for the given constants $\tilde{\beta}, T_{2}, N_{1}$ and $B$, one can choose $M_{1}>0$ large enough such that

$$
\int_{-\infty}^{-N_{1}}\left(u_{l}^{\nu}-\tilde{u}\right)\left(y, T_{2}\right) d y=-\frac{M_{1}}{\tilde{\beta}} e^{-\tilde{\beta}\left(N_{1}+s T_{2}\right)}<-B .
$$

Now we claim that

Claim 1.

$$
\tilde{U}\left(x, T_{2}\right)=\int_{-\infty}^{x}\left(u^{\nu}-\tilde{u}\right)\left(y, T_{2}\right) d y \leqslant \varepsilon \quad \forall x \in \mathbb{R} .
$$

In fact, if $x \leqslant-N_{1}$, then (3.45) implies that

$$
\int_{-\infty}^{x}\left(u^{\nu}-\tilde{u}\right)\left(y, T_{2}\right) d y \leqslant \int_{-\infty}^{x}\left(u^{\nu}-u_{l}^{\nu}\right)\left(y, T_{2}\right) d y \leqslant \varepsilon .
$$

And if $x>-N_{1}$, it follows from (3.45) and (3.46) that

$$
\begin{aligned}
\int_{-\infty}^{x}\left(u^{\nu}-\tilde{u}\right)\left(y, T_{2}\right) d y= & \int_{-\infty}^{-N_{1}}\left(u^{\nu}-u_{l}^{\nu}\right)+\int_{-\infty}^{-N_{1}}\left(u_{l}^{\nu}-\tilde{u}\right) \\
& +\int_{-N_{1}}^{x}\left(u^{\nu}-u_{r}^{\nu}\right)+\int_{-N_{1}}^{x}\left(u_{r}^{\nu}-\tilde{u}\right) \\
\leqslant & \varepsilon-B+B+0=\varepsilon,
\end{aligned}
$$

which proves Claim 1 .

Combing (3.44) with Claim 1, and using the maximum principle [16, Lemma 1], one can obtain that

$$
\tilde{U}(x, t) \leqslant \varepsilon \quad \forall x \in \mathbb{R}, t \geqslant T_{2},
$$


which implies that

$$
U_{l}^{\nu}(x, t)=\int_{-\infty}^{x}\left(u^{\nu}-u_{l}^{\nu}\right)(y, t) d y=\tilde{U}(x, t)+\frac{M_{1}}{\tilde{\beta}} e^{\tilde{\beta}(x-s t)} \leqslant 2 \varepsilon,
$$

if $t \geqslant T_{2}$ and $x-s t<-N_{1}$ with $N_{1}>0$ large enough.

Step 2. In this step, it is aimed to construct a "sub-solution" $\psi_{\xi}^{\nu}$ to (1.1) such that the anti-derivative variable of $u^{\nu}-\psi_{\xi}^{\nu}$ has the lower bound $-\varepsilon$ (see (3.55)). The idea is to find a $C^{\infty}$ curve $\xi(t):\left[T_{2},+\infty\right) \rightarrow \mathbb{R}$ such that the ansatz $\psi_{\xi}^{\nu}$ defined by $(2.8)$ satisfies that

$$
h_{\xi}^{\nu}=\partial_{t} \psi_{\xi}^{\nu}+\partial_{x} f\left(\psi_{\xi}^{\nu}\right)-\nu \partial_{x}^{2} \psi_{\xi}^{\nu}<0 \quad \forall x \in \mathbb{R}, t>T_{2},
$$

with the proper initial data (3.53).

For two given constants $M_{2}>0$ and $d>0$, where $M_{2}$ will be determined in this step, and $d$ will be determined in the next step, we define a $C^{\infty}$ curves $\xi(t)$ : $\left[T_{2},+\infty\right) \rightarrow \mathbb{R}$, which solves the following problem:

$$
\left\{\begin{array}{l}
\xi^{\prime}(t)=s-M_{2} e^{-\alpha t}, \quad t>T_{2}, \\
\xi\left(T_{2}\right)=-d .
\end{array}\right.
$$

Then by (3.49), there exists a constant $D>d$, depending on $\alpha, T_{2}, d$ and $M_{2}$, such that

$$
\xi(t) \in[s t-D, s t+D] \quad \forall t \geqslant T_{2} .
$$

Now we calculate the source term $h_{\xi}^{\nu}$. It follows from (2.12) and (2.13) that for $t \geqslant T_{2}$,

$$
\begin{aligned}
h_{\xi}^{\nu}= & \left(u_{l}^{\nu}-u_{r}^{\nu}\right)\left(\xi^{\prime}(t)-s+f^{\prime}\left(\phi_{\xi}^{\nu}\right)-f^{\prime}\left(\psi_{\xi}^{\nu}\right)+2 \nu \frac{\partial_{x} u_{l}^{\nu}-\partial_{x} u_{r}^{\nu}}{u_{l}^{\nu}-u_{r}^{\nu}}\right)\left|\left(g_{\xi}^{\nu}\right)^{\prime}\right| \\
& -\left(u_{l}^{\nu}-u_{r}^{\nu}\right)\left(b\left(\psi_{\xi}^{\nu}, u_{l}^{\nu}\right) \partial_{x} u_{l}^{\nu}-b\left(\psi_{\xi}^{\nu}, u_{r}^{\nu}\right) \partial_{x} u_{r}^{\nu}\right) g_{\xi}^{\nu}\left(1-g_{\xi}^{\nu}\right),
\end{aligned}
$$

where $b(u, v)$ is defined in (3.38). Then by Lemma 2.1 and (2.4), one has that for $t \geqslant T_{2}$,

$$
\begin{aligned}
h_{\xi}^{\nu} \leqslant & \left(u_{l}^{\nu}-u_{r}^{\nu}\right)\left(\xi^{\prime}(t)-s+f^{\prime}\left(\phi_{\xi}^{\nu}\right)-f^{\prime}\left(\psi_{\xi}^{\nu}\right)+2 \nu \frac{\partial_{x} u_{l}^{\nu}-\partial_{x} u_{r}^{\nu}}{u_{l}^{\nu}-u_{r}^{\nu}}\right)\left|\left(g_{\xi}^{\nu}\right)^{\prime}\right| \\
& +C\left(u_{l}^{\nu}-u_{r}^{\nu}\right)\left(\left|\partial_{x} u_{r}^{\nu}\right|+\left|\partial_{x} u_{l}^{\nu}\right|\right)\left|\left(g_{\xi}^{\nu}\right)^{\prime}\right| \\
\leqslant & \left(u_{l}^{\nu}-u_{r}^{\nu}\right)\left|\left(g_{\xi}^{\nu}\right)^{\prime}\right|\left[-M_{2} e^{-\alpha t}+C\left|\phi_{\xi}^{\nu}-\psi_{\xi}^{\nu}\right|+C\left(\left|\partial_{x} u_{r}^{\nu}\right|+\left|\partial_{x} u_{l}^{\nu}\right|\right)\right] .
\end{aligned}
$$

Note that

$$
\left|\phi_{\xi}^{\nu}-\psi_{\xi}^{\nu}\right| \leqslant\left|\bar{u}_{l}-u_{l}^{\nu}\right| g_{\xi}^{\nu}+\left|\bar{u}_{r}-u_{r}^{\nu}\right|\left(1-g_{\xi}^{\nu}\right) \leqslant C e^{-\alpha t} \quad \forall x \in \mathbb{R} .
$$

Then if $M>0$ is large enough, it holds that

$$
h_{\xi}^{\nu} \leqslant\left(u_{l}^{\nu}-u_{r}^{\nu}\right)\left|\left(g_{\xi}^{\nu}\right)^{\prime}\right|\left(-M_{2}+C\right) e^{-\alpha t}<0 \quad \forall x \in \mathbb{R}, t>T_{2} .
$$

Therefore, for $M_{2}>0$ large enough, (3.48) is fulfilled with the $\xi$ constructed in (3.49).

Step 3. In this step, we will prove that there exists $N_{2}=N_{2}(\varepsilon)>0$ such that

$$
U_{l}^{\nu}(x, t)>-\varepsilon, \quad t>T_{2}, x<s t-N_{2} .
$$


For the constants $N_{1}$ and $B$ defined in (3.45) and (3.46), one can choose the constant $d=\xi\left(T_{2}\right)>0$ (which is in (3.49)) large enough such that

$$
\begin{aligned}
& \int_{-\infty}^{-N_{1}}\left(u_{l}^{\nu}-\psi_{-d}^{\nu}\right)\left(x, T_{2}\right) d x+\int_{-N_{1}}^{+\infty}\left(u_{r}^{\nu}-\psi_{-d}^{\nu}\right)\left(x, T_{2}\right) d x \\
= & \int_{-\infty}^{-N_{1}}\left(u_{l}^{\nu}-u_{r}^{\nu}\right)\left(x, T_{2}\right)\left(1-g^{\nu}(x+d)\right) d x-\int_{-N_{1}}^{+\infty}\left(u_{l}^{\nu}-u_{r}^{\nu}\right)\left(x, T_{2}\right) g^{\nu}(x+d) d x \\
> & B .
\end{aligned}
$$

Then we claim that

Claim 2.

$$
\check{U}_{\xi\left(T_{2}\right)}^{\nu}\left(x, T_{2}\right)=\int_{-\infty}^{x}\left(u^{\nu}-\psi_{-d}^{\nu}\right)\left(y, T_{2}\right) d y \geqslant-\varepsilon \quad \forall x \in \mathbb{R} .
$$

where $\check{U_{\xi}^{\nu}}$ is defined in $(3.21)$.

In fact, if $x \leqslant-N_{1}$, then it follows from (3.45) that

$$
\int_{-\infty}^{x}\left(u^{\nu}-\psi_{-d}^{\nu}\right)\left(y, T_{2}\right) d y \geqslant \int_{-\infty}^{x}\left(u^{\nu}-u_{l}^{\nu}\right)\left(y, T_{2}\right) d y \geqslant-\varepsilon
$$

And if $x>-N_{1}$, then by (3.45), (3.46) and (3.52), one can get that

$$
\begin{aligned}
\int_{-\infty}^{x}\left(u^{\nu}-\psi_{-d}^{\nu}\right) & =\int_{-\infty}^{-N_{1}}\left(u^{\nu}-u_{l}^{\nu}\right)+\int_{-\infty}^{-N_{1}}\left(u_{l}^{\nu}-\psi_{-d}^{\nu}\right)+\int_{-N_{1}}^{x}\left(u^{\nu}-u_{r}^{\nu}\right)+\int_{-N_{1}}^{x}\left(u_{r}^{\nu}-\psi_{-d}^{\nu}\right) \\
& \geqslant-\varepsilon+\int_{-\infty}^{-N_{1}}\left(u_{l}^{\nu}-\psi_{-d}^{\nu}\right)-B+\int_{-N_{1}}^{+\infty}\left(u_{r}^{\nu}-\psi_{-d}^{\nu}\right) \\
& \geqslant-\varepsilon .
\end{aligned}
$$

Thus Claim 2 is proved.

It can follow from Lemma 3.3 that

$$
\partial_{t} \check{U}_{\xi}^{\nu}-\nu \partial_{x}^{2} \check{U}_{\xi}^{\nu}=f\left(\psi_{\xi}^{\nu}\right)-f\left(u^{\nu}\right)-\int_{-\infty}^{x} h_{\xi}^{\nu}(y, t) d y \quad \forall x \in \mathbb{R}, t \geqslant T_{2} .
$$

This and (3.48) yield that

$$
\partial_{t} \check{U}_{\xi}^{\nu}-\nu \partial_{x}^{2} \check{U}_{\xi}^{\nu}+a\left(u^{\nu}, \psi_{\xi}^{\nu}\right) \partial_{x} \check{U}_{\xi}^{\nu}>0 \quad \forall x \in \mathbb{R}, t \geqslant T_{2},
$$

where $a$ is defined by (3.37). Then by (3.53), (3.54), and using the maximum principle, one can obtain

$$
\check{U}_{\xi}^{\nu}(x, t) \geqslant-\varepsilon \quad \forall x \in \mathbb{R}, t \geqslant T_{2} .
$$

This, together with (2.13) and (3.50), shows that

$$
\begin{aligned}
U_{l}^{\nu}(x, t) & =\int_{-\infty}^{x}\left(u^{\nu}-u_{l}^{\nu}\right)(y, t) d y \\
& =\int_{-\infty}^{x}\left(u^{\nu}-\psi_{\xi}^{\nu}\right)(y, t) d y+\int_{-\infty}^{x}\left(\psi_{\xi}^{\nu}-u_{l}^{\nu}\right)(y, t) d y \\
& =\check{U}_{\xi}^{\nu}(x, t)-\int_{-\infty}^{x}\left(u_{l}^{\nu}-u_{r}^{\nu}\right)(y, t)\left[1-g^{\nu}(y-\xi(t))\right] d y \\
& \geqslant-\varepsilon-2\left(\bar{u}_{l}-\bar{u}_{r}\right) \int_{-\infty}^{x}\left[1-g^{\nu}(y-s t+D)\right] d y
\end{aligned}
$$




$$
\geqslant-2 \varepsilon
$$

provided that $t \geqslant T_{2}$ and $x-s t<-N_{2}$ with $N_{2}>0$ large enough.

Step 4. In the last step, we complete the proof of (3.39).

By Steps 1 and 3, for any $t>T_{2}$ and $x_{1}<x_{2}<s t-N_{2}$, one has that

$$
\left|\int_{x_{1}}^{x_{2}}\left(u^{\nu}-u_{l}^{\nu}\right)(y, t) d y\right| \leqslant 2 \varepsilon .
$$

And by (1.4) and Lemma 2.4, there exists a positive number $M_{3}>0$ such that

$$
\partial_{x}\left(u^{\nu}-u_{l}^{\nu}\right) \leqslant M_{3} \quad \forall t>T_{2}, x \in \mathbb{R} .
$$

Then (3.39) follows from the following Claim.

Claim 3. For any $t>T_{2}, x<s t-N_{2}-\frac{3 \sqrt{\varepsilon}}{\sqrt{M_{3}}}$, it holds that

$$
\left|u^{\nu}(x, t)-u_{l}^{\nu}(x, t)\right| \leqslant 3 \sqrt{M_{3} \varepsilon} .
$$

In fact, if there exist $t_{0}>T_{2}$ and $x_{0}<s t_{0}-N_{2}-\frac{3 \sqrt{\varepsilon}}{\sqrt{M_{3}}}$ such that

$$
u^{\nu}\left(x_{0}, t_{0}\right)-u_{l}^{\nu}\left(x_{0}, t_{0}\right)<-3 \sqrt{M_{3} \varepsilon}
$$

then for any $x \in\left(x_{0}, x_{0}+\frac{3 \sqrt{\varepsilon}}{\sqrt{M_{3}}}\right)$, it holds that

$$
\left(u^{\nu}-u_{l}^{\nu}\right)\left(x, t_{0}\right)-\left(u^{\nu}-u_{l}^{\nu}\right)\left(x_{0}, t_{0}\right)=\partial_{x}\left(u^{\nu}-u_{l}^{\nu}\right)\left(\cdot, t_{0}\right)\left(x-x_{0}\right) .
$$

Due to (3.57), it follows from above that

$$
\left(u^{\nu}-u_{l}^{\nu}\right)\left(x, t_{0}\right)<-3 \sqrt{M_{3} \varepsilon}+M_{3}\left(x-x_{0}\right) .
$$

Then integrating this inequality over $\left(x_{0}, x_{0}+\frac{3 \sqrt{\varepsilon}}{\sqrt{M_{3}}}\right)$ yields that

$$
\int_{x_{0}}^{x_{0}+\frac{3 \sqrt{\varepsilon}}{\sqrt{M_{3}}}}\left(u^{\nu}-u_{l}^{\nu}\right)\left(x, t_{0}\right) d x \leqslant-3 \sqrt{M_{3} \varepsilon} \times \frac{3 \sqrt{\varepsilon}}{\sqrt{M_{3}}}+\frac{M_{3}}{2} \times \frac{9 \varepsilon}{M_{3}}=-\frac{9}{2} \varepsilon<-2 \varepsilon,
$$

which contradicts (3.56). For the other case that $u^{\nu}\left(x_{0}, t_{0}\right)-u_{l}^{\nu}\left(x_{0}, t_{0}\right)>3 \sqrt{M_{3} \varepsilon}$, it is also a contradiction by considering the interval $\left(x_{0}-\frac{3 \sqrt{\varepsilon}}{\sqrt{M_{3}}}, x_{0}\right)$ instead. So Claim 3 holds true.

\subsubsection{Anti-derivative variables.}

In this part, we consider the equation of the anti-derivative of $u^{\nu}-\psi_{X^{\nu}}^{\nu}$. It turns out that the error term in the equation for the anti-derivative variable decays exponentially both in space and in time; see Proposition 3.5. And then the idea of Il'in and Olě̌nik [16] is applied to proving (2.19), i.e. we construct an auxiliary function $(\Theta(x)$ constructed below), and then use the maximal principle.

We first define the anti-derivative variable of $-h_{X^{\nu}}^{\nu}$ in (2.9) as

$$
H^{\nu}(x, t):=-\int_{-\infty}^{x} h_{X^{\nu}(t)}^{\nu}(y, t) d y, \quad t \geqslant T_{0}, x \in \mathbb{R}, .
$$


Then due to (2.11), one has that

$$
\begin{aligned}
H^{\nu}= & -\left(f\left(\psi_{X^{\nu}}^{\nu}\right)-f\left(u_{l}^{\nu}\right)\right) g_{X^{\nu}}^{\nu}-\left(f\left(\psi_{X^{\nu}}^{\nu}\right)-f\left(u_{r}^{\nu}\right)\right)\left(1-g_{X^{\nu}}^{\nu}\right) \\
& +2 \nu\left(u_{l}^{\nu}-u_{r}^{\nu}\right)\left(g_{X^{\nu}}^{\nu}\right)^{\prime}-\int_{-\infty}^{x}\left(f\left(u_{l}^{\nu}\right)-f\left(u_{r}^{\nu}\right)\right)(y, t)\left(g_{X^{\nu}}^{\nu}\right)^{\prime}(y) d y \\
& +\left(X^{\nu}\right)^{\prime} \int_{-\infty}^{x}\left(u_{l}^{\nu}-u_{r}^{\nu}\right)(y, t)\left(g_{X^{\nu}}^{\nu}\right)^{\prime}(y) d y \\
& -\nu \int_{-\infty}^{x}\left(u_{l}^{\nu}-u_{r}^{\nu}\right)(y, t)\left(g_{X^{\nu}}^{\nu}\right)^{\prime \prime}(y) d y .
\end{aligned}
$$

On the other hand, by Lemma 3.2, one can define the anti-derivative variable of $u^{\nu}-\psi_{X^{\nu}}^{\nu}$ as:

$$
U^{\nu}(x, t):=\check{U}_{X^{\nu}}^{\nu}(x, t)=\int_{-\infty}^{x}\left(u^{\nu}-\psi_{X^{\nu}}^{\nu}\right)(y, t) d y, \quad t \geqslant 0, x \in \mathbb{R} .
$$

For convenience, in the following part of this paper we define

$$
\bar{a}(x, t):=a\left(u^{\nu}, \psi_{X^{\nu}}^{\nu}\right)(x, t) .
$$

Proposition 3.5. The functions $H^{\nu}(x, t)$ and $\bar{a}(x, t)$ are smooth in $\mathbb{R} \times\left[T_{0},+\infty\right)$, and $U^{\nu}(x, t)$ solves the equation

$$
\partial_{t} U^{\nu}-\nu \partial_{x}^{2} U^{\nu}+\bar{a} \partial_{x} U^{\nu}=H^{\nu}, \quad x \in \mathbb{R}, t \geqslant T_{0},
$$

where $a(u, v)$ is defined by (3.37), and all the derivatives of $U^{\nu}$ appearing in (3.63) are continuous in $\mathbb{R} \times\left[T_{0},+\infty\right)$. Moreover, $H^{\nu}(x, t)$ satisfies

$$
\left|H^{\nu}(x, t)\right| \leqslant C_{1} e^{-\alpha t} e^{-\beta\left|x-X^{\nu}(t)\right|} \quad \forall x \in \mathbb{R}, t \geqslant T_{0},
$$

and $U^{\nu}(x, t)$ satisfies

$$
\left|U^{\nu}(x, t)\right| \leqslant C_{2}(t) e^{-\beta\left|x-X^{\nu}(t)\right|} \quad \forall x \in \mathbb{R}, t \geqslant T_{0},
$$

where $C_{1}>0$ is a constant, independent of time $t$, and $C_{2}(t)>0$ is bounded on any compact subset of $\left[T_{0},+\infty\right)$.

Proof. The smoothness of $H^{\nu}(x, t)$ and $\bar{a}(x, t)$ can be derived easily from Lemma 2.4 and the smoothness of $u^{\nu}, u_{l}^{\nu}, u_{r}^{\nu}$ and $\psi_{X^{\nu}}^{\nu}$. By Lemma 3.3, $U^{\nu}=\check{U}_{X^{\nu}}^{\nu}$ solves (3.63) and all the derivatives of $U^{\nu}$ appearing in (3.63) are continuous in $\mathbb{R} \times\left[T_{0},+\infty\right)$.

Now we prove (3.64). Note that there exists a unique $x_{0} \in \mathbb{R}$ such that, if $x<x_{0}$, $\left(g^{\nu}\right)^{\prime \prime}(x)<0$, and if $x>x_{0},\left(g^{\nu}\right)^{\prime \prime}(x)>0$. Then for $x<X^{\nu}(t)+x_{0}$, it follows from (3.60) and Lemma 2.4 that

$$
\begin{aligned}
& H^{\nu}(x, t)=-\left(f\left(\psi_{X^{\nu}}^{\nu}\right)-f\left(u_{l}^{\nu}\right)\right)-\left(f\left(\bar{u}_{l}\right)-f\left(\bar{u}_{r}\right)+O\left(e^{-\alpha t}\right)\right)\left(1-g_{X^{\nu}}^{\nu}\right) \\
& \quad+2 \nu\left(\bar{u}_{l}-\bar{u}_{r}+O\left(e^{-\alpha t}\right)\right)\left(g_{X^{\nu}}^{\nu}\right)^{\prime}-\int_{-\infty}^{x}\left(f\left(\bar{u}_{l}\right)-f\left(\bar{u}_{r}\right)+O\left(e^{-\alpha t}\right)\right)\left(g_{X^{\nu}}^{\nu}\right)^{\prime}(y) d y \\
& \quad+\left(s+O\left(e^{-\alpha t}\right)\right) \int_{-\infty}^{x}\left(\bar{u}_{l}-\bar{u}_{r}+O\left(e^{-\alpha t}\right)\right)\left(g_{X^{\nu}}^{\nu}\right)^{\prime}(y) d y \\
& \quad-\nu \int_{-\infty}^{x}\left(\bar{u}_{l}-\bar{u}_{r}+O\left(e^{-\alpha t}\right)\right)\left(g_{X^{\nu}}^{\nu}\right)^{\prime \prime}(y) d y .
\end{aligned}
$$

If $x<X^{\nu}(t)+x_{0}, \int_{-\infty}^{x}\left|\left(g_{X^{\nu}}^{\nu}\right)^{\prime \prime}\right|=-\int_{-\infty}^{x}\left(g_{X^{\nu}}^{\nu}\right)^{\prime \prime}=-\left(g_{X^{\nu}}^{\nu}\right)^{\prime}$, and $1-g_{X^{\nu}}^{\nu}(x)+\left|\left(g_{X^{\nu}}^{\nu}\right)^{\prime}(x)\right|=$ $O\left(e^{\beta\left(x-X^{\nu}(t)\right)}\right)$, then

$$
H^{\nu}(x, t)=-\left(f\left(\psi_{X^{\nu}}^{\nu}\right)-f\left(u_{l}^{\nu}\right)\right)-\left(f\left(\bar{u}_{l}\right)-f\left(\bar{u}_{r}\right)\right)\left(1-g_{X^{\nu}}^{\nu}\right)+2 \nu\left(\bar{u}_{l}-\bar{u}_{r}\right)\left(g_{X^{\nu}}^{\nu}\right)^{\prime}
$$




$$
\begin{aligned}
& +\left(f\left(\bar{u}_{l}\right)-f\left(\bar{u}_{r}\right)\right)\left(1-g_{X^{\nu}}^{\nu}\right)-s\left(\bar{u}_{l}-\bar{u}_{r}\right)\left(1-g_{X^{\nu}}^{\nu}\right) \\
& -\nu\left(\bar{u}_{l}-\bar{u}_{r}\right)\left(g_{X^{\nu}}^{\nu}\right)^{\prime}+O\left(e^{-\alpha t} e^{\beta\left(x-X^{\nu}(t)\right)}\right) \\
= & -\left(f\left(\psi_{X^{\nu}}^{\nu}\right)-f\left(u_{l}^{\nu}\right)\right)+\left(f\left(\phi_{X^{\nu}}^{\nu}\right)-f\left(\bar{u}_{l}\right)\right) \\
= & -a\left(\psi_{X^{\nu}}^{\nu}, u_{l}^{\nu}\right)\left(\psi_{X^{\nu}}^{\nu}-u_{l}^{\nu}\right)+a\left(\phi_{X^{\nu}}^{\nu}, \bar{u}_{l}\right)\left(\phi_{X^{\nu}}^{\nu}-\bar{u}_{l}\right) \\
= & a\left(\psi_{X^{\nu}}^{\nu}, u_{l}^{\nu}\right)\left(u_{l}^{\nu}-u_{r}^{\nu}\right)\left(1-g_{X^{\nu}}^{\nu}\right)-a\left(\phi_{X^{\nu}}^{\nu}, \bar{u}_{l}\right)\left(\bar{u}_{l}-\bar{u}_{r}\right)\left(1-g_{X^{\nu}}^{\nu}\right) \\
= & \left(\bar{u}_{l}-\bar{u}_{r}\right)\left(1-g_{X^{\nu}}^{\nu}\right)\left(a\left(\psi_{X^{\nu}}^{\nu}, u_{l}^{\nu}\right)-a\left(\phi_{X^{\nu}}^{\nu}, \bar{u}_{l}\right)\right)+O\left(e^{-\alpha t} e^{\beta\left(x-X^{\nu}(t)\right)}\right) .
\end{aligned}
$$

And it is easy to verify that

$$
a\left(\psi_{X^{\nu}}^{\nu}, \bar{u}_{l}\right)-a\left(\phi_{X^{\nu}}^{\nu}, u_{l}^{\nu}\right)=O\left(e^{-\alpha t}\right)
$$

which implies that if $x<X^{\nu}(t)+x_{0}, H^{\nu}(x, t)=O\left(e^{-\alpha t} e^{\beta\left(x-X^{\nu}(t)\right)}\right)$. On the other hand, if $x>X^{\nu}(t)+x_{0}, \int_{x}^{+\infty}\left|\left(g_{X^{\nu}}^{\nu}\right)^{\prime \prime}\right|=\int_{x}^{+\infty}\left(g_{X^{\nu}}^{\nu}\right)^{\prime \prime}=-\left(g_{X^{\nu}}^{\nu}\right)^{\prime}$, and $g_{X^{\nu}}^{\nu}(x)+\left|\left(g_{X^{\nu}}^{\nu}\right)^{\prime}(x)\right|=$ $O\left(e^{-\beta\left(x-X^{\nu}(t)\right)}\right)$. It follows from (2.15) that for any $t>T_{0}$ and $x \in \mathbb{R}$,

$$
\begin{aligned}
H^{\nu}(x, t)= & -\left(f\left(\psi_{X^{\nu}}^{\nu}\right)-f\left(u_{l}^{\nu}\right)\right) g_{X^{\nu}}^{\nu}-\left(f\left(\psi_{X^{\nu}}^{\nu}\right)-f\left(u_{r}^{\nu}\right)\right)\left(1-g_{X^{\nu}}^{\nu}\right) \\
& +2 \nu\left(u_{l}^{\nu}-u_{r}^{\nu}\right)\left(g_{X^{\nu}}^{\nu}\right)^{\prime}+\int_{x}^{+\infty}\left(f\left(u_{l}^{\nu}\right)-f\left(u_{r}^{\nu}\right)\right)(y, t)\left(g_{X^{\nu}}^{\nu}\right)^{\prime}(y) d y \\
& -\left(X^{\nu}\right)^{\prime}(t) \int_{x}^{+\infty}\left(u_{l}^{\nu}-u_{r}^{\nu}\right)(y, t)\left(g_{X^{\nu}}^{\nu}\right)^{\prime}(y) d y \\
& +\nu \int_{x}^{+\infty}\left(u_{l}^{\nu}-u_{r}^{\nu}\right)(y, t)\left(g_{X^{\nu}}^{\nu}\right)^{\prime \prime}(y) d y .
\end{aligned}
$$

Then by similar arguments as above, one can prove that if $x<X^{\nu}(t)+x_{0}$, $H^{\nu}(x, t)=O\left(e^{-\alpha t} e^{-\beta\left(x-X^{\nu}(t)\right)}\right)$. Hence, one can get (3.64).

Now we will prove (3.65). Similar to the proof of (3.5), one can apply (2.15) and (2.17) to obtain that

$$
\int_{-\infty}^{+\infty}\left(u^{\nu}(x, t)-\psi_{X^{\nu}(t)}^{\nu}(x, t)\right) d x=0 \quad \forall t \geqslant T_{0}
$$

Hence, for any $x \in \mathbb{R}$ and $t>0$, one has that

$$
U^{\nu}(x, t)=\int_{-\infty}^{x}\left(u^{\nu}-\psi_{X^{\nu}}^{\nu}\right)(y, t) d y=-\int_{x}^{+\infty}\left(u^{\nu}-\psi_{X^{\nu}}^{\nu}\right)(y, t) d y
$$

Lemma 3.1 implies that $\left|X^{\nu}(t)\right| \leqslant C+|s| t$. Then by Lemma 3.2, one has that

$$
\left|u^{\nu}(x, t)-\psi_{X^{\nu}}^{\nu}(x, t)\right| \leqslant C(t) e^{\beta\left|X^{\nu}(t)\right|} e^{-\beta\left|x-X^{\nu}(t)\right|} \leqslant C_{2}(t) e^{-\beta\left|x-X^{\nu}(t)\right|},
$$

which, together with (3.67), yields (3.65).

Denote the positive constant $\varepsilon_{1}:=\min \left\{\frac{f^{\prime}\left(\bar{u}_{l}\right)-s}{2},-\frac{f^{\prime}\left(\bar{u}_{r}\right)-s}{2}\right\}>0$.

Lemma 3.6. There exist positive constants $T_{3}>T_{2}$ and $N_{0}$, independent of time, such that

$$
\begin{aligned}
& \bar{a}(x, t)-\left(X^{\nu}\right)^{\prime}(t)>\varepsilon_{1}>0, \quad x-X^{\nu}(t)<-N_{0}, t>T_{3}, \\
& \bar{a}(x, t)-\left(X^{\nu}\right)^{\prime}(t)<-\varepsilon_{1}<0, \quad x-X^{\nu}(t)>N_{0}, t>T_{3} .
\end{aligned}
$$


Proof. Here we prove (3.68) only, since (3.69) can be proved similarly.

By $(3.37), \bar{a}(x, t)=\int_{0}^{1} f^{\prime}\left(\psi_{X^{\nu}}^{\nu}+\rho\left(u^{\nu}-\psi_{X^{\nu}}^{\nu}\right)\right) d \rho$, where

$$
\begin{aligned}
& \left|\psi_{X^{\nu}}^{\nu}+\rho\left(u^{\nu}-\psi_{X^{\nu}}^{\nu}\right)-\bar{u}_{l}\right| \\
= & \left|u_{l}^{\nu} g_{X^{\nu}}^{\nu}+u_{r}^{\nu}\left(1-g_{X^{\nu}}^{\nu}\right)+\rho\left(u^{\nu}-u_{l}^{\nu}\right)+\rho\left(u_{l}^{\nu}-u_{r}^{\nu}\right)\left(1-g_{X^{\nu}}^{\nu}\right)-\bar{u}_{l}\right| \\
= & \left|\left(u_{l}^{\nu}-\bar{u}_{l}\right)-(1-\rho)\left(u_{l}^{\nu}-u_{r}^{\nu}\right)\left(1-g_{X^{\nu}}^{\nu}\right)+\rho\left(u^{\nu}-u_{l}^{\nu}\right)\right| \\
\leqslant & \left|u_{l}^{\nu}-\bar{u}_{l}\right|+2\left(\bar{u}_{l}-\bar{u}_{r}\right)\left(1-g_{X^{\nu}}^{\nu}\right)+\left|u^{\nu}-u_{l}^{\nu}\right| .
\end{aligned}
$$

Lemma 3.1 shows that $\left|X^{\nu}(t)-s t\right| \leqslant C$. Then by combining Lemmas 2.1 and 2.4 with Proposition 3.4, one can get that for any $\varepsilon>0$, there exist $T(\varepsilon)>T_{2}$ and $N(\varepsilon)>0$ such that

$$
\left|\psi_{X^{\nu}}^{\nu}+\rho\left(u^{\nu}-\psi_{X^{\nu}}^{\nu}\right)-\bar{u}_{l}\right|<\varepsilon \quad \forall t>T(\varepsilon), x-X^{\nu}(t)<-N(\varepsilon),
$$

and hence,

$$
\left|f^{\prime}\left(\psi_{X^{\nu}}^{\nu}+\rho\left(u^{\nu}-\psi_{X^{\nu}}^{\nu}\right)\right)-f^{\prime}\left(\bar{u}_{l}\right)\right| \leqslant C \varepsilon \quad \forall t>T(\varepsilon), x-X^{\nu}(t)<-N(\varepsilon) .
$$

Then it follows from Lemma 3.1 that

$$
\begin{aligned}
\bar{a}-\left(X^{\nu}\right)^{\prime} & >f^{\prime}\left(\bar{u}_{l}\right)-C \varepsilon-s+\left[s-\left(X^{\nu}\right)^{\prime}(t)\right] \\
& \geqslant f^{\prime}\left(\bar{u}_{l}\right)-C \varepsilon-s-C e^{-\alpha t} \\
& \geqslant \frac{f^{\prime}\left(\bar{u}_{l}\right)-s}{2} \quad \forall t>T_{3}, x-X^{\nu}(t)<-N_{0},
\end{aligned}
$$

provided that $\varepsilon$ is small enough, and $N_{0}>N(\varepsilon)$ and $T_{3}>T(\varepsilon)$ are large enough.

Define the linear operator $L$ as

$$
L:=\partial_{t}-\nu \partial_{x}^{2}+\bar{a}(x, t) \partial_{x} .
$$

Therefore, Proposition 3.5 shows that $L U^{\nu}=H^{\nu}$ on $\left\{t \geqslant T_{0}\right\}$. For the constant $N_{0}$ given in Lemma 3.6, we can define a convex $C^{2}$ function $\theta$ on $\mathbb{R}$ and the auxiliary function $\Theta$ as [16]:

$$
\begin{aligned}
& \theta(x):= \begin{cases}\cosh (\gamma x), & |x| \leqslant N_{0}, \\
\theta \in C^{2}, 0 \leqslant \theta^{\prime \prime} \leqslant \gamma^{2} \cosh (\gamma x), & N_{0}<|x| \leqslant N_{0}+1, \\
\text { linear function, } & |x|>N_{0}+1,\end{cases} \\
& \Theta(x):=e^{-\delta \theta(x)},
\end{aligned}
$$

where $\gamma$ and $\delta$ are two positive constants to be determined.

Lemma 3.7. There exist positive constants $\gamma, \delta$ and $\mu$, independent of time $t$, such that the auxiliary function $\Theta$ defined above satisfies that

$$
L\left(\Theta\left(x-X^{\nu}(t)\right)\right) \geqslant 2 \mu \Theta\left(x-X^{\nu}(t)\right) \quad \forall x \in \mathbb{R}, t>T_{3} .
$$

Proof. In the following we define $\zeta:=x-X^{\nu}(t)$ for simplicity.

By (3.70), it holds that

$$
\begin{aligned}
L(\Theta(\zeta)) & =e^{-\delta \theta(\zeta)}\left[\delta\left(X^{\nu}\right)^{\prime}(t) \theta^{\prime}(\zeta)-\delta^{2} \nu\left(\theta^{\prime}(\zeta)\right)^{2}+\delta \nu \theta^{\prime \prime}(\zeta)-\delta \bar{a} \theta^{\prime}(\zeta)\right] \\
& =\delta \Theta(\zeta)\left[\nu \theta^{\prime \prime}(\zeta)-\left(\bar{a}(x, t)-\left(X^{\nu}\right)^{\prime}(t)\right) \theta^{\prime}(\zeta)-\delta \nu\left(\theta^{\prime}(\zeta)\right)^{2}\right]
\end{aligned}
$$

There are three cases to be considered. 
(1) If $|\zeta|<N_{0}, \theta(\zeta)=\cosh (\gamma \zeta)$. Therefore,

$$
\begin{aligned}
& \nu \theta^{\prime \prime}(\zeta)-\left(\bar{a}(x, t)-\left(X^{\nu}\right)^{\prime}(t)\right) \theta^{\prime}(\zeta)-\delta \nu\left(\theta^{\prime}(\zeta)\right)^{2} \\
= & \nu \gamma^{2} \cosh (\gamma \zeta)-\left(\bar{a}-\left(X^{\nu}\right)^{\prime}(t)\right) \gamma \sinh (\gamma \zeta)-\nu \delta \gamma^{2}(\sinh (\gamma \zeta))^{2} \\
= & \gamma \cosh (\gamma \zeta)\left[\nu \gamma(1-\delta \sinh (\gamma \zeta) \tanh (\gamma \zeta))-\left(\bar{a}-\left(X^{\nu}\right)^{\prime}(t)\right) \tanh (\gamma \zeta)\right] .
\end{aligned}
$$

Since $\bar{a}-\left(X^{\nu}\right)^{\prime}$ is bounded, one can first choose $\gamma>0$ large enough such that

$$
\left|\bar{a}-\left(X^{\nu}\right)^{\prime}\right|<\frac{\nu \gamma}{4} \quad \forall x \in \mathbb{R}, t>0 .
$$

For $|\zeta|<N_{0},|\sinh (\gamma \zeta)|<e^{\gamma N_{0}}$, then one can choose $\delta=\delta\left(\gamma, N_{0}\right)>0$ small enough such that

$$
1-\delta e^{\gamma N_{0}} \geqslant \frac{1}{2}
$$

Hence, by $|\tanh (\gamma \zeta)| \leqslant 1$ and $\cosh (\gamma \zeta) \geqslant 1$, it holds that for $|\zeta|<N_{0}$ and $t>T_{3}$,

$$
L(\Theta(\zeta)) \geqslant \delta \Theta(\zeta) \cdot \gamma \cosh (\gamma \zeta) \cdot \frac{\nu \gamma}{4} \geqslant \frac{\delta \nu \gamma^{2}}{4} \Theta(\zeta) .
$$

(2) If $\zeta>N_{0}$, by $0 \leqslant \theta^{\prime \prime}(\zeta) \leqslant \gamma^{2} \cosh (\gamma \zeta)$, it holds that $0<k_{1} \leqslant \theta^{\prime}(\zeta) \leqslant k_{2}$, where $k_{1}=\gamma \sinh \left(\gamma N_{0}\right)$ and $k_{2}=\gamma \sinh \left(\gamma\left(N_{0}+1\right)\right)$. It follows from the fact $\theta^{\prime \prime} \geqslant 0$ and Lemma 3.6 that

$$
\begin{aligned}
& \nu \theta^{\prime \prime}(\zeta)-\left(\bar{a}-\left(X^{\nu}\right)^{\prime}(t)\right) \theta^{\prime}(\zeta)-\delta \nu\left(\theta^{\prime}(\zeta)\right)^{2} \\
\geqslant & -\left(\bar{a}-\left(X^{\nu}\right)^{\prime}\right) \theta^{\prime}(\zeta)-\delta \nu\left(\theta^{\prime}(\zeta)\right)^{2} \\
\geqslant & \theta^{\prime}(\zeta)\left(\varepsilon_{1}-\delta \nu \theta^{\prime}(\zeta)\right)
\end{aligned}
$$

One can choose $\delta=\delta\left(\gamma, N_{0}, \varepsilon_{1}\right)>0$ small enough such that for $\zeta>N_{0}$,

$$
\delta \nu \theta^{\prime}(\zeta) \leqslant \delta \nu k_{2} \leqslant \frac{\varepsilon_{1}}{2} .
$$

Hence,

$$
L(\Theta(\zeta)) \geqslant \delta \Theta(\zeta) \cdot k_{1} \cdot \frac{\varepsilon_{1}}{2} \geqslant \frac{\delta \varepsilon_{1} k_{1}}{2} \Theta(\zeta)
$$

(3) For the case $\zeta<-N_{0}$, (3.72) can be proved similarly.

Collecting (1), (2) and (3), one can prove the lemma by choosing $\gamma$ sufficiently large, $\delta$ sufficiently small and $\mu=\min \left\{\frac{\delta \nu \gamma^{2}}{8}, \frac{\delta \varepsilon_{1} k_{1}}{4}\right\}$.

\subsubsection{Proof of Proposition 2.\%.}

Set

$$
Z(x, t):=M_{2} e^{-\mu t} \Theta\left(x-X^{\nu}(t)\right) \pm U^{\nu}(x, t), \quad x \in \mathbb{R}, t \geqslant 0,
$$

where $M_{2}>0$ is a constant to be determined, and $\mu$ is the constant in Lemma 3.7, which can be actually chosen small enough with $0<\mu \leqslant \min \{1, \alpha\}$. Due to (3.64) and Lemma 3.7, it holds that

$$
\begin{aligned}
L Z & =-\mu M_{2} e^{-\mu t} \Theta\left(x-X^{\nu}(t)\right)+M_{2} e^{-\mu t} L \Theta\left(x-X^{\nu}(t)\right) \pm H^{\nu} \\
& \geqslant(-\mu+2 \mu) M_{2} e^{-\mu t} \Theta\left(x-X^{\nu}(t)\right)-C_{0} e^{-\alpha t} e^{-\beta\left|x-X^{\nu}(t)\right|} \\
& \geqslant e^{-\mu t}\left(\mu M_{2} e^{-\delta \theta\left(x-X^{\nu}(t)\right)}-C_{0} e^{-\beta\left|x-X^{\nu}(t)\right|}\right) .
\end{aligned}
$$


By (3.65), one has

$$
Z\left(x, T_{3}\right) \geqslant M_{2} e^{-\mu T_{3}} e^{-\delta \theta\left(x-X^{\nu}\left(T_{3}\right)\right)}-C\left(T_{3}\right) e^{-\beta\left|x-X^{\nu}\left(T_{3}\right)\right|} .
$$

We need to consider two cases:

(1) If $\left|x-X^{\nu}(t)\right| \leqslant N_{0}+1$, then

$$
\begin{gathered}
\mu M_{2} e^{-\delta \theta\left(x-X^{\nu}(t)\right)}-C_{0} e^{-\beta\left|x-X^{\nu}(t)\right|} \geqslant \mu M_{2} e^{-\delta \theta\left(N_{0}+1\right)}-C_{0}, \\
M_{2} e^{-\mu T_{3}} e^{-\delta \theta\left(x-X^{\nu}\left(T_{3}\right)\right)}-C\left(T_{3}\right) e^{-\beta\left|x-X^{\nu}\left(T_{3}\right)\right|} \geqslant M_{2} e^{-\mu T_{3}} e^{-\delta \theta\left(N_{0}+1\right)}-C\left(T_{3}\right)
\end{gathered}
$$

Therefore, choosing $M_{2}>\max \left\{\frac{C_{0}}{\mu} e^{\delta \theta\left(N_{0}+1\right)}, C\left(T_{3}\right) e^{\mu T_{3}} e^{\delta \theta\left(N_{0}+1\right)}\right\}$, one gets that $L Z>0$ and $Z\left(x, T_{3}\right)>0$.

(2) If $\left|x-X^{\nu}(t)\right|>N_{0}+1$, then $\theta$ is linear and

$$
\left|\theta^{\prime}\left(x-X^{\nu}(t)\right)\right|<k_{2}=\gamma \sinh \left(\gamma\left(N_{0}+1\right)\right) .
$$

Therefore, by $\theta(x) \leqslant \cosh (x)$ for $|x|>N_{0}+1$, it holds that

$$
\theta\left(x-X^{\nu}(t)\right)<k_{2}\left|x-X^{\nu}(t)\right|+\cosh \left(\gamma\left(N_{0}+1\right)\right) .
$$

Then one can get that

$$
\begin{aligned}
& \mu M_{2} e^{-\delta \theta\left(x-X^{\nu}(t)\right)}-C_{0} e^{-\beta\left|x-X^{\nu}(t)\right|} \\
& \quad \geqslant \mu M_{2} e^{-\delta \cosh \left(\gamma\left(N_{0}+1\right)\right)} e^{-\delta k_{2}\left|x-X^{\nu}(t)\right|}-C_{0} e^{-\beta\left|x-X^{\nu}(t)\right|}, \\
& M_{2} e^{-\mu T_{3}} e^{-\delta \theta\left(x-X^{\nu}\left(T_{3}\right)\right)}-C\left(T_{3}\right) e^{-\beta\left|x-X^{\nu}\left(T_{3}\right)\right|} \\
& \quad \geqslant M_{2} e^{-\mu T_{3}} e^{-\delta \cosh \left(\gamma\left(N_{0}+1\right)\right)} e^{-\delta k_{2}\left|x-X^{\nu}\left(T_{3}\right)\right|}-C\left(T_{3}\right) e^{-\beta\left|x-X^{\nu}\left(T_{3}\right)\right|}
\end{aligned}
$$

Then by choosing $\delta$ small enough with $\delta k_{2} \leqslant \beta$, and $M_{2}$ large enough with

$$
M_{2}>\max \left\{\frac{C_{0}}{\mu} e^{\delta \cosh \left(\gamma\left(N_{0}+1\right)\right)}, C\left(T_{3}\right) e^{\mu T_{3}} e^{\delta \cosh \left(\gamma\left(N_{0}+1\right)\right)}\right\},
$$

one can also obtain that $L Z>0$ and $Z\left(x, T_{3}\right)>0$.

By combining (1) with (2), one gets that if $\delta$ is small and $M_{2}$ is large, $L Z>0$ and $Z\left(x, T_{3}\right)>0$ for any $x \in \mathbb{R}$ and $t \geqslant T_{3}$. Therefore, the maximum principle implies that $Z(x, t) \geqslant 0$ for any $x \in \mathbb{R}, t \geqslant T_{3}$, which yields that

$$
\left|U^{\nu}(x, t)\right| \leqslant M_{2} e^{-\mu t} \Theta\left(x-X^{\nu}(t)\right) \leqslant M_{2} e^{-\mu t} \quad \forall x \in \mathbb{R}, t \geqslant T_{3} .
$$

Hence, by the definition (3.61) of $U^{\nu}$, one has that for any $x_{1}<x_{2}$ and $t \geqslant T_{3}$,

$$
\left|\int_{x_{1}}^{x_{2}}\left(u^{\nu}(y, t)-\psi_{X^{\nu}}^{\nu}(y, t)\right) d y\right|=\left|U^{\nu}\left(x_{2}, t\right)-U^{\nu}\left(x_{1}, t\right)\right| \leqslant 2 M_{2} e^{-\mu t} .
$$

By (1.4) and Lemmas 2.1 and 2.4, there exists a constant $M_{3}>0$, independent of time $t$, such that for any $x \in \mathbb{R}$ and $t \geqslant T_{3}$,

(3.74) $\partial_{x}\left(u^{\nu}-\psi_{X^{\nu}}^{\nu}\right)=\partial_{x}\left(u^{\nu}-u_{l}^{\nu}\right) g_{X^{\nu}}^{\nu}+\partial_{x}\left(u^{\nu}-u_{r}^{\nu}\right)\left(1-g_{X^{\nu}}^{\nu}\right)-\left(u_{l}^{\nu}-u_{r}^{\nu}\right)\left(g_{X^{\nu}}^{\nu}\right)^{\prime} \leqslant M_{3}$.

It then follows from (3.73) and (3.74) that the following claim holds true.

Claim 5 .

$$
\left|u^{\nu}(x, t)-\psi_{X^{\nu}}^{\nu}(x, t)\right| \leqslant 3 \sqrt{M_{2} M_{3}} e^{-\frac{\mu}{2} t} \quad \forall x \in \mathbb{R}, t \geqslant T_{3} .
$$

Indeed, if there exists $\left(x_{0}, t_{0}\right)$ with $x_{0} \in \mathbb{R}$ and $t_{0} \geqslant T_{3}$, such that

$$
u^{\nu}\left(x_{0}, t_{0}\right)-\psi_{X^{\nu}}^{\nu}\left(x_{0}, t_{0}\right)<-3 \sqrt{M_{2} M_{3}} e^{-\frac{\mu}{2} t_{0}} .
$$


Then for any $x \in\left(x_{0}, x_{1}\right)$, where $x_{1}:=x_{0}+3 \sqrt{\frac{M_{2}}{M_{3}}} e^{-\frac{\mu}{2} t_{0}},(3.74)$ yields that

$$
\left(u^{\nu}\left(x, t_{0}\right)-\psi_{X^{\nu}}^{\nu}\left(x, t_{0}\right)\right)-\left(u^{\nu}\left(x_{0}, t_{0}\right)-\psi_{X^{\nu}}^{\nu}\left(x_{0}, t_{0}\right)\right) \leqslant M_{3}\left(x-x_{0}\right) .
$$

Then

$$
\begin{aligned}
& \int_{x_{0}}^{x_{1}}\left(u^{\nu}\left(x, t_{0}\right)-\psi_{X^{\nu}}^{\nu}\left(x, t_{0}\right)\right) d x \\
\leqslant & \left(u^{\nu}\left(x_{0}, t_{0}\right)-\psi_{X^{\nu}}^{\nu}\left(x_{0}, t_{0}\right)\right)\left(x_{1}-x_{0}\right)+\frac{M_{3}}{2}\left(x_{1}-x_{0}\right)^{2} \\
\leqslant & -3 \sqrt{M_{2} M_{3}} e^{-\frac{\mu}{2} t_{0}} \cdot 3 \sqrt{\frac{M_{2}}{M_{3}}} e^{-\frac{\mu}{2} t_{0}}+\frac{M_{3}}{2} \cdot 9 \frac{M_{2}}{M_{3}} e^{-\mu t_{0}} \\
= & -\frac{9}{2} M_{2} e^{-\mu t_{0}}<-2 M_{2} e^{-\mu t_{0}},
\end{aligned}
$$

which contradicts $(3.73)$. In the other case for $u^{\nu}\left(x_{0}, t_{0}\right)-\psi_{X^{\nu}}^{\nu}\left(x_{0}, t_{0}\right)>3 \sqrt{M_{2} M_{3}} e^{-\frac{\mu}{2} t_{0}}$ at some point $\left(x_{0}, t_{0}\right)$, a contradiction can be obtained similarly by considering the interval $\left(x_{0}-3 \sqrt{\frac{M_{2}}{M_{3}}} e^{-\frac{\mu}{2} t_{0}}, x_{0}\right)$ instead.

Therefore, the claim above is proved. This, together with the fact that $u^{\nu}$ and $\psi_{X^{\nu}}^{\nu}$ are both bounded, yields (2.19).

\subsection{More results for Burgers' equation.}

In this section, we prove the result (1) in Theorem 1.2 and Proposition 2.8 for the Burgers' equation (1.1), where $f(u)=u^{2} / 2$ and the two periodic perturbations are the same.

First, under the assumptions of Theorem 1.2, one can use the Galilean transformation to verify that the periodic functions $w_{l}^{\nu}$ and $w_{r}^{\nu}$ defined in (3.11) satisfy

$$
w_{l}^{\nu}(x, t)=w_{r}^{\nu}\left(x-\left(\bar{u}_{l}-\bar{u}_{r}\right) t, t\right), \quad x \in \mathbb{R}, t \geqslant 0,
$$

Therefore, it holds that for any $t>0$,

$$
\begin{aligned}
& \int_{0}^{t} \int_{0}^{p}\left\{\left[f\left(u_{l}^{\nu}\right)-f\left(\bar{u}_{l}\right)\right]-\left[f\left(u_{r}^{\nu}\right)-f\left(\bar{u}_{r}\right)\right]\right\} d x d \tau \\
= & \int_{0}^{t} \int_{0}^{p} \frac{1}{2}\left\{\left[2 \bar{u}_{l}+w_{l}^{\nu}(x, \tau)\right] w_{l}^{\nu}(x, \tau)-\left[2 \bar{u}_{r}+w_{r}^{\nu}(x, \tau)\right] w_{r}^{\nu}(x, \tau)\right\} d x d \tau \\
= & \int_{0}^{t} \int_{0}^{p} \frac{1}{2}\left[\left(w_{l}^{\nu}\right)^{2}(x, \tau)-\left(w_{r}^{\nu}\right)^{2}(x, \tau)\right] d x d \tau \\
= & \int_{0}^{t} \int_{0}^{p} \frac{1}{2}\left(w_{r}^{\nu}\right)^{2}\left(x-\left(\bar{u}_{l}-\bar{u}_{r}\right) t, \tau\right) d x d \tau-\int_{0}^{t} \int_{0}^{p} \frac{1}{2}\left(w_{r}^{\nu}\right)^{2}(x, \tau) d x d \tau \\
= & \int_{0}^{t} \int_{-\left(\bar{u}_{l}-\bar{u}_{r}\right) t}^{p-\left(\bar{u}_{l}-\bar{u}_{r}\right) t} \frac{1}{2}\left(w_{r}^{\nu}\right)^{2}(x, \tau) d x d \tau-\int_{0}^{t} \int_{0}^{p} \frac{1}{2}\left(w_{r}^{\nu}\right)^{2}(x, \tau) d x d \tau=0,
\end{aligned}
$$

where the second equality holds since the averages of $w_{l}^{\nu}$ and $w_{r}^{\nu}$ are zero. Thus, letting $t \rightarrow+\infty$ in (3.76) shows that $X_{\infty, 2}^{\nu}$ defined in (1.11) is identically zero. 
Now it remains to prove Proposition 2.8. Under the assumptions of Proposition 2.8, it holds that $u_{l}^{\nu}(x, t) \geqslant u_{r}^{\nu}(x, t)$ for all $x \in \mathbb{R}$ and $t>0$, thus

$$
\begin{aligned}
\int_{\mathbb{R}}\left(u_{l}^{\nu}(x, t)-u_{r}^{\nu}(x, t)\right)\left(g_{\xi}^{\nu}\right)^{\prime}(x) d x & =\int_{0}^{p}\left(u_{l}^{\nu}(x, t)-u_{r}^{\nu}(x, t)\right) \sum_{k \in \mathbb{Z}}\left(g_{\xi}^{\nu}\right)^{\prime}(x+k p) d x \\
& \leqslant-C \int_{0}^{p}\left(u_{l}^{\nu}(x, t)-u_{r}^{\nu}(x, t)\right) \sum_{k \in \mathbb{Z}} e^{-\beta(x+k p)} d x \\
& \leqslant-C e^{-\beta p} \int_{0}^{p}\left(u_{l}^{\nu}(x, t)-u_{r}^{\nu}(x, t)\right) d x \\
& =-C e^{-\beta p} p\left(\bar{u}_{l}-\bar{u}_{r}\right)<0 .
\end{aligned}
$$

Then the number $M$ in (3.2) can be chosen to be zero. Moreover, since $M=0$ and the initial data given in Proposition 2.8 satisfies $\int_{\mathbb{R}}\left(u_{0}(x)-\psi_{0}^{\nu}(x)\right) d x=0$, the unique number $\hat{X}_{0}^{\nu}$ satisfying (3.5) is zero. Hence, one can get that the curve $\tilde{X}^{\nu}$ defined in (3.8) actually solves

$$
\left\{\begin{array}{l}
\left(\tilde{X}^{\nu}\right)^{\prime}(t)=F^{\nu}\left(\tilde{X}^{\nu}, t\right), \quad t>0 \\
\tilde{X}^{\nu}(0)=0
\end{array}\right.
$$

Due to $(3.75)$, if $\left(\bar{u}_{l}-\bar{u}_{r}\right) t_{k}=k p$ for any $k \geqslant 0$, one has $w_{l}^{\nu}\left(x, t_{k}\right) \equiv w_{r}^{\nu}\left(x, t_{k}\right)$. Thus the term $J^{N}$ defined in (3.12) and the limit $J$ satisfy that

$$
J^{N}\left(y, t_{k}\right) \equiv 0 \quad \Rightarrow \quad J\left(y, t_{k}\right)=\lim _{N \rightarrow+\infty} J^{N}\left(y, t_{k}\right) \equiv 0 .
$$

Then taking (3.76), $M=\hat{X}_{0}^{\nu}=0$, and (3.78) into (3.17) implies that $\tilde{X}^{\nu}\left(t_{k}\right)=s t_{k}$, and thus (2.22) holds true.

It remains to prove (2.21). In [14], Hopf introduced the well known Hopf transformation to obtain an explicit formula for the solution to (1.1) with any initial data $u_{0} \in L^{\infty}$, which is given by:

$$
u^{\nu}(x, t)=\frac{\int_{\mathbb{R}} \frac{x-y}{t} \exp \left\{-\frac{(x-y)^{2}}{4 \nu t}-\frac{1}{2 \nu} \int_{0}^{y} u_{0}(\eta) d \eta\right\} d y}{\int_{\mathbb{R}} \exp \left\{-\frac{(x-y)^{2}}{4 \nu t}-\frac{1}{2 \nu} \int_{0}^{y} u_{0}(\eta) d \eta\right\} d y}, \quad x \in \mathbb{R}, t>0 .
$$

Since $u_{0}$ is bounded, then integration by parts on the numerator of (3.79) yields that

$$
u^{\nu}(x, t)=\frac{\int_{\mathbb{R}} u_{0}(y) \exp \left\{-\frac{(x-y)^{2}}{4 \nu t}-\frac{1}{2 \nu} \int_{0}^{y} u_{0}(\eta) d \eta\right\} d y}{\int_{\mathbb{R}} \exp \left\{-\frac{(x-y)^{2}}{4 \nu t}-\frac{1}{2 \nu} \int_{0}^{y} u_{0}(\eta) d \eta\right\} d y}, \quad x \in \mathbb{R}, t>0 .
$$

Without loss of generality (the viscous shock profile is unique up to a shift), the viscous shock $\phi^{\nu}$ connecting the end states $\bar{u}_{l}$ at $x=-\infty$ and $\bar{u}_{r}$ at $x=+\infty$ to the Burgers' equation can be given by the explicit formula

$$
\phi^{\nu}(x)=\frac{\bar{u}_{l}+\bar{u}_{r}}{2}-\frac{\bar{u}_{l}-\bar{u}_{r}}{2} \tanh \left(\frac{\bar{u}_{l}-\bar{u}_{r}}{4 \nu} x\right) .
$$

Set $\lambda:=\frac{\bar{u}_{l}-\bar{u}_{r}}{4 \nu}$ for convenience. Then the associated $g^{\nu}$ defined in (2.3) is given by

$$
g^{\nu}(x)=\frac{1-\tanh (\lambda x)}{2}=\frac{e^{-\lambda x}}{e^{\lambda x}+e^{-\lambda x}},
$$

satisfying

$$
\phi^{\nu}(x)=\bar{u}_{l} g^{\nu}(x)+\bar{u}_{r}\left(1-g^{\nu}(x)\right)
$$


And one also has that

$$
\begin{aligned}
\int_{0}^{x} g^{\nu}(y) d y & =\frac{1}{2} \int_{0}^{x}[1-\tanh (\lambda y)] d y=\frac{1}{2 \lambda} \log \frac{e^{\lambda x}}{e^{\lambda x}+e^{-\lambda x}}+\frac{1}{2 \lambda} \log 2 \\
& =\frac{1}{2 \lambda} \log \left(1-g^{\nu}(x)\right)+\frac{1}{2 \lambda} \log 2 .
\end{aligned}
$$

Similarly,

$$
\int_{0}^{x}\left[1-g^{\nu}(y)\right] d y=-\frac{1}{2 \lambda} \log g^{\nu}(x)-\frac{1}{2 \lambda} \log 2 .
$$

If the initial data $u_{0}(x)=\phi^{\nu}(x)+w_{0}(x)$ with $\int_{0}^{p} w_{0}(x) d x=0$, then it follows from (3.80) and (3.82) that

$$
u^{\nu}(x, t)=\frac{P_{l}(x, t)+P_{r}(x, t)}{Q_{l}(x, t)+Q_{r}(x, t)}
$$

where the two terms in the numerator are

$$
\begin{aligned}
& P_{l}(x, t):=2 \int_{\mathbb{R}}\left(\bar{u}_{l}+w_{0}(y)\right) g^{\nu}(y) \exp \left\{-\frac{(x-y)^{2}}{4 \nu t}-\frac{1}{2 \nu} \int_{0}^{y} u_{0}(\eta) d \eta\right\} d y, \\
& P_{r}(x, t):=2 \int_{\mathbb{R}}\left(\bar{u}_{r}+w_{0}(y)\right)\left(1-g^{\nu}(y)\right) \exp \left\{-\frac{(x-y)^{2}}{4 \nu t}-\frac{1}{2 \nu} \int_{0}^{y} u_{0}(\eta) d \eta\right\} d y,
\end{aligned}
$$

and the two terms in the denominator are

$$
\begin{aligned}
& Q_{l}(x, t):=2 \int_{\mathbb{R}} g^{\nu}(y) \exp \left\{-\frac{(x-y)^{2}}{4 \nu t}-\frac{1}{2 \nu} \int_{0}^{y} u_{0}(\eta) d \eta\right\} d y \\
& Q_{r}(x, t):=2 \int_{\mathbb{R}}\left(1-g^{\nu}(y)\right) \exp \left\{-\frac{(x-y)^{2}}{4 \nu t}-\frac{1}{2 \nu} \int_{0}^{y} u_{0}(\eta) d \eta\right\} d y .
\end{aligned}
$$

It can follow from (3.84), $\lambda=\frac{\bar{u}_{l}-\bar{u}_{r}}{4 \nu}$, and $u_{0}=\bar{u}_{l} g^{\nu}+\bar{u}_{r}\left(1-g^{\nu}\right)+w_{0}=\bar{u}_{l}+w_{0}-$ $\left(\bar{u}_{l}-\bar{u}_{r}\right)\left(1-g^{\nu}\right)$ that

$$
\begin{aligned}
& g^{\nu}(y) \exp \left\{-\frac{1}{2 \nu} \int_{0}^{y} u_{0}(\eta) d \eta\right\} \\
= & g^{\nu}(y) \exp \left\{2 \lambda \int_{0}^{y}\left(1-g^{\nu}(\eta)\right) d \eta\right\} \exp \left\{-\frac{1}{2 \nu} \int_{0}^{y}\left(\bar{u}_{l}+w_{0}(\eta)\right) d \eta\right\} \\
= & \frac{1}{2} \exp \left\{-\frac{1}{2 \nu} \int_{0}^{y}\left(\bar{u}_{l}+w_{0}(\eta)\right) d \eta\right\} .
\end{aligned}
$$

Due to (3.83), similar calculations yield

$$
\left(1-g^{\nu}(y)\right) \exp \left\{-\frac{1}{2 \nu} \int_{0}^{y} u_{0}(\eta) d \eta\right\}=\frac{1}{2} \exp \left\{-\frac{1}{2 \nu} \int_{0}^{y}\left(\bar{u}_{r}+w_{0}(\eta)\right) d \eta\right\}
$$


Hence, one has that

$$
\begin{aligned}
& P_{l}(x, t)=\int_{\mathbb{R}}\left(\bar{u}_{l}+w_{0}(y)\right) \exp \left\{-\frac{(x-y)^{2}}{4 \nu t}-\frac{1}{2 \nu} \int_{0}^{y}\left(\bar{u}_{l}+w_{0}(\eta)\right) d \eta\right\} d y, \\
& P_{r}(x, t)=\int_{\mathbb{R}}\left(\bar{u}_{r}+w_{0}(y)\right) \exp \left\{-\frac{(x-y)^{2}}{4 \nu t}-\frac{1}{2 \nu} \int_{0}^{y}\left(\bar{u}_{r}+w_{0}(\eta)\right) d \eta\right\} d y, \\
& Q_{l}(x, t)=\int_{\mathbb{R}} \exp \left\{-\frac{(x-y)^{2}}{4 \nu t}-\frac{1}{2 \nu} \int_{0}^{y}\left(\bar{u}_{l}+w_{0}(\eta)\right) d \eta\right\} d y, \\
& Q_{r}(x, t)=\int_{\mathbb{R}} \exp \left\{-\frac{(x-y)^{2}}{4 \nu t}-\frac{1}{2 \nu} \int_{0}^{y}\left(\bar{u}_{r}+w_{0}(\eta)\right) d \eta\right\} d y .
\end{aligned}
$$

Moreover, by using the Hopf formula (3.80) for $u_{l}^{\nu}(x, t)$ and $u_{r}^{\nu}(x, t)$, respectively, one gets that

$$
u_{l}^{\nu}(x, t)=\frac{P_{l}(x, t)}{Q_{l}(x, t)} \quad \text { and } \quad u_{r}^{\nu}(x, t)=\frac{P_{r}(x, t)}{Q_{r}(x, t)} .
$$

If $t=t_{k}=\frac{k p}{\bar{u}_{l}-\bar{u}_{r}}=\frac{k p}{4 \nu \lambda}$, it holds that

$$
\begin{aligned}
Q_{r}\left(x, t_{k}\right)= & \int_{\mathbb{R}} \exp \left\{-\frac{(x-y)^{2}}{4 \nu t_{k}}+\frac{1}{2 \nu} \int_{0}^{y} 4 \nu \lambda d \eta-\frac{1}{2 \nu} \int_{0}^{y}\left(\bar{u}_{l}+w_{0}(\eta)\right) d \eta\right\} d y \\
= & e^{2 \lambda x+4 \lambda^{2} \nu t_{k}} \int_{\mathbb{R}} \exp \left\{-\frac{\left(y-x-4 \lambda \nu t_{k}\right)^{2}}{4 \nu t_{k}}-\frac{1}{2 \nu} \int_{0}^{y}\left(\bar{u}_{l}+w_{0}(\eta)\right) d \eta\right\} d y \\
= & e^{2 \lambda x+4 \lambda^{2} \nu t_{k}} \int_{\mathbb{R}} \exp \left\{-\frac{(y-x)^{2}}{4 \nu t_{k}}-\frac{1}{2 \nu} \int_{0}^{y+4 \lambda \nu t_{k}}\left(\bar{u}_{l}+w_{0}(\eta)\right) d \eta\right\} d y \\
= & e^{2 \lambda x+4 \lambda^{2} \nu t_{k}-2 \lambda \bar{u}_{l} t_{k}} \int_{\mathbb{R}} \exp \left\{-\frac{(y-x)^{2}}{4 \nu t_{k}}-\frac{1}{2 \nu} \int_{0}^{y}\left(\bar{u}_{l}+w_{0}(\eta)\right) d \eta\right\} \\
& \times \exp \left\{-\frac{1}{2 \nu} \int_{y}^{y+4 \lambda \nu t_{k}} w_{0}(\eta) d \eta\right\} d y .
\end{aligned}
$$

Then due to $\int_{0}^{p} w_{0}(x) d x=0$ and $4 \lambda \nu t_{k}=k p$, it holds that

$$
Q_{r}\left(x, t_{k}\right)=e^{2 \lambda\left(x-s t_{k}\right)} Q_{l}\left(x, t_{k}\right), \quad x \in \mathbb{R} .
$$

Similarly, one can get that

$$
P_{r}\left(x, t_{k}\right)=e^{2 \lambda\left(x-s t_{k}\right)} P_{l}\left(x, t_{k}\right)-4 \lambda e^{2 \lambda\left(x-s t_{k}\right)} Q_{l}\left(x, t_{k}\right), \quad x \in \mathbb{R} .
$$

Hence, (3.85) yields that

$$
\begin{aligned}
u^{\nu}\left(x, t_{k}\right) & =\frac{P_{l}\left(x, t_{k}\right)+e^{2 \lambda\left(x-s t_{k}\right)} P_{l}\left(x, t_{k}\right)-4 \lambda e^{2 \lambda\left(x-s t_{k}\right)} Q_{l}\left(x, t_{k}\right)}{Q_{l}\left(x, t_{k}\right)+e^{2 \lambda\left(x-s t_{k}\right)} Q_{l}\left(x, t_{k}\right)} \\
& =\frac{P_{l}\left(x, t_{k}\right)}{Q_{l}\left(x, t_{k}\right)}-4 \lambda\left(1-g^{\nu}\left(x-s t_{k}\right)\right)
\end{aligned}
$$

where $g^{\nu}$ is defined in (3.81). Meanwhile, (3.86) yields that

$$
u_{l}^{\nu}\left(x, t_{k}\right)=\frac{P_{l}\left(x, t_{k}\right)}{Q_{l}\left(x, t_{k}\right)} \text { and } u_{r}^{\nu}\left(x, t_{k}\right)=\frac{P_{l}\left(x, t_{k}\right)-4 \lambda Q_{l}\left(x, t_{k}\right)}{Q_{l}\left(x, t_{k}\right)}=\frac{P_{l}\left(x, t_{k}\right)}{Q_{l}\left(x, t_{k}\right)}-4 \lambda,
$$

which, together with (3.87), yields (2.21). 


\subsection{An example of non-zero shift.}

In this section, we prove the result (2) in Theorem 1.2, where the two periodic perturbations are assumed to be the same. For any given periodic perturbation $w_{0}$ with zero average and $0<\left\|w_{0}\right\|_{L^{\infty}(\mathbb{R})}<\left(\bar{u}_{l}-\bar{u}_{r}\right) / 2$, it holds that $\bar{u}_{r}+\left\|w_{0}\right\|_{L^{\infty}(\mathbb{R})}<$ $\bar{u}_{l}-\left\|w_{0}\right\|_{L^{\infty}(\mathbb{R})}$, then one can construct a smooth and strictly convex function $f$ such that $f(u)=\frac{1}{2 n} u^{2}$ when $u \leqslant \bar{u}_{r}+\left\|w_{0}\right\|_{L^{\infty}(\mathbb{R})}$ and $f(u)=\frac{1}{2} u^{2}$ when $u \geqslant \bar{u}_{l}-\left\|w_{0}\right\|_{L^{\infty}(\mathbb{R})}$, where $n$ is a positive number to be determined later; see Figure 2.

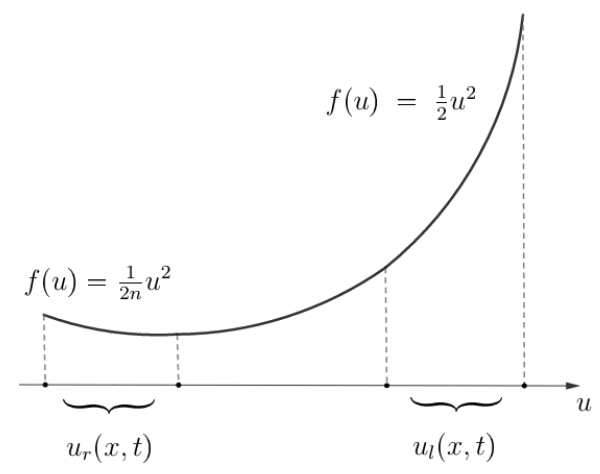

Figure 2. Construction of $f$

Since for any $x \in \mathbb{R}$ and $t>0$, it holds that

$$
\begin{aligned}
& u_{l}^{\nu}(x, t) \geqslant \inf u_{l}^{\nu}(\cdot, 0) \geqslant \bar{u}_{l}-\left\|w_{0}\right\|_{L^{\infty}(\mathbb{R})}, \\
& u_{r}^{\nu}(x, t) \leqslant \sup u_{r}^{\nu}(\cdot, 0) \leqslant \bar{u}_{r}+\left\|w_{0}\right\|_{L^{\infty}(\mathbb{R})} .
\end{aligned}
$$

And note that $w_{l}^{\nu}=u_{l}^{\nu}-\bar{u}_{l}$ and $w_{r}^{\nu}=u_{r}^{\nu}-\bar{u}_{r}$ have zero average, then one has that

$$
\begin{aligned}
\int_{0}^{\infty} \int_{0}^{p}\left[f\left(u_{l}^{\nu}\right)-f\left(\bar{u}_{l}\right)\right] d x d \tau & =\frac{1}{2} \int_{0}^{\infty} \int_{0}^{p}\left[\left(u_{l}^{\nu}\right)^{2}-\bar{u}_{l}^{2}\right] d x d \tau \\
& =\frac{1}{2} \int_{0}^{\infty} \int_{0}^{p}\left[\left(\bar{u}_{l}+w_{l}^{\nu}\right)^{2}-\bar{u}_{l}^{2}\right] d x d \tau \\
& =\frac{1}{2} \int_{0}^{\infty} \int_{0}^{p}\left(w_{l}^{\nu}\right)^{2} d x d \tau .
\end{aligned}
$$

Similarly,

$$
\begin{aligned}
\int_{0}^{\infty} \int_{0}^{p}\left[f\left(u_{r}^{\nu}\right)-f\left(\bar{u}_{r}\right)\right] d x d \tau & =\frac{1}{2 n} \int_{0}^{\infty} \int_{0}^{p}\left[\left(u_{r}^{\nu}\right)^{2}-\bar{u}_{r}^{2}\right] d x d \tau \\
& =\frac{1}{2 n} \int_{0}^{\infty} \int_{0}^{p}\left(w_{r}^{\nu}\right)^{2} d x d \tau .
\end{aligned}
$$

Since $w_{0}$ is not identically zero, the solution $u_{l}^{\nu}(x, t)$ with the initial data $\bar{u}_{l}+w_{0}(x)$ cannot be a constant in $\mathbb{R} \times[0,+\infty)$, thus the integral of (3.88) is positive. And more importantly, this integral is independent of $n$, since no matter what $n$ is, the range of $u_{l}^{\nu}(x, t)$ is always in the interval where $f(u)$ is $u^{2} / 2$, which means that $u_{l}^{\nu}(x, t)$ is actually a solution to the Burgers' equation.

On the other side, for the solution $u_{r}^{\nu}(x, t),(\mathrm{A} .2)$ yields that

$$
\int_{0}^{+\infty} \int_{0}^{p}\left(w_{r}^{\nu}\right)^{2} d x d \tau \leqslant C
$$


where $C$ is independent of $f$, depending only on $\nu, p$ and $\left\|u_{r}^{\nu}(x, 0)\right\|_{L^{\infty}}$. It follows from this and (3.89) that

$$
\int_{0}^{\infty} \int_{0}^{p}\left[f\left(u_{r}^{\nu}\right)-f\left(\bar{u}_{r}\right)\right] d x d \tau \leqslant \frac{C}{2 n} .
$$

By (3.88) and (3.90), one can choose $n$ sufficiently large such that

$$
\int_{0}^{\infty} \int_{0}^{p}\left[f\left(u_{l}^{\nu}\right)-f\left(\bar{u}_{l}\right)\right] d x d \tau>\int_{0}^{\infty} \int_{0}^{p}\left[f\left(u_{r}^{\nu}\right)-f\left(\bar{u}_{r}\right)\right] d x d \tau
$$

which implies that $X_{\infty, 2}^{\nu} \neq 0$. The proof of Theorem 1.2 is finished.

\subsection{Vanishing viscosity limit for the shift.}

We now study the vanishing viscosity limit for $X_{\infty, 2}^{\nu}$ defined in (1.11).

Lemma 3.8. There exists a constant $C>0$, independent of time $t$ or viscosity $\nu$, such that for all $0 \leqslant \nu \leqslant 1$, it holds that

$$
\sup _{x \in \mathbb{R}}\left|u_{l}^{\nu}-\bar{u}_{l}\right|+\sup _{x \in \mathbb{R}}\left|u_{r}^{\nu}-\bar{u}_{r}\right| \leqslant \frac{C}{1+t}, \quad t>0 .
$$

Proof. The proof can be found in Xin [30], which relies on the Oleǐnik's entropy condition (1.4). For $i=l$ or $r$, it follows from $\int_{0}^{p_{i}} \partial_{x} u_{i}^{\nu}(x, t) d x=0$ and (1.4) that for any $0<\nu \leqslant 1$ and $t>0$,

$$
\int_{x \in\left(0, p_{i}\right), \partial_{x} u_{i}^{\nu}<0}\left|\partial_{x} u_{i}^{\nu}(x, t)\right| d x=\int_{x \in\left(0, p_{i}\right), \partial_{x} u_{i}^{\nu}>0} \partial_{x} u_{i}^{\nu}(x, t) d x \leqslant \frac{p_{i} E}{t},
$$

which yields that for any $0<\nu \leqslant 1$ and $t>0$,

$$
\sup _{x}\left|u_{i}^{\nu}-\bar{u}_{l}\right| \leqslant \int_{0}^{p_{i}}\left|\partial_{x} u_{i}^{\nu}(x, t)\right| d x \leqslant \frac{2 p_{i} E}{t} .
$$

Since $u_{l}^{\nu}$ and $u_{r}^{\nu}$ converges almost everywhere to the periodic entropy solutions $u_{l}^{0}$ and $u_{r}^{0}$, respectively, (3.91) also holds true for $\nu=0$.

Proof of Theorem 1.3. It follows from Taylor's expansion and zero average of $u_{l}^{\nu}-\bar{u}_{l}$ that

$$
\begin{aligned}
\int_{0}^{p_{l}}\left[f\left(u_{l}^{\nu}\right)-f\left(\bar{u}_{l}\right)\right] d x & =\int_{0}^{p_{l}}\left(f^{\prime}\left(\bar{u}_{l}\right)\left(u_{l}^{\nu}-\bar{u}_{l}\right)+\frac{1}{2} f^{\prime \prime}(\cdot)\left(u_{l}^{\nu}-\bar{u}_{l}\right)^{2}\right) d x \\
& =\frac{1}{2} \int_{0}^{p} f^{\prime \prime}(\cdot)\left(u_{l}^{\nu}-\bar{u}_{l}\right)^{2} d x .
\end{aligned}
$$

This, together with Lemma 3.8 and the strict convexity of $f$, implies that

$$
0<\int_{0}^{p_{l}}\left[f\left(u_{l}^{\nu}\right)-f\left(\bar{u}_{l}\right)\right] d x \leqslant \frac{C}{(1+t)^{2}},
$$

where $C$ is independent of $\nu$ or $t$. Similarly, one has that

$$
0<\int_{0}^{p_{r}}\left[f\left(u_{r}^{\nu}\right)-f\left(\bar{u}_{r}\right)\right] d x \leqslant \frac{C}{(1+t)^{2}} .
$$


Hence, applying the dominated convergence theorem in (1.11) yields that, as $\nu \rightarrow 0+$,

$$
\begin{aligned}
X_{\infty, 2}^{\nu} \rightarrow & \int_{0}^{+\infty} \frac{1}{p_{l}} \int_{0}^{p_{l}}\left[f\left(u_{l}^{0}\right)-f\left(\bar{u}_{l}\right)\right] d x d t-\frac{1}{p_{l}} \int_{0}^{p_{l}} \int_{0}^{x} w_{0 l}(y) d y d x \\
& -\int_{0}^{+\infty} \frac{1}{p_{l}} \int_{0}^{p_{r}}\left[f\left(u_{r}^{0}\right)-f\left(\bar{u}_{r}\right)\right] d x d t+\frac{1}{p_{r}} \int_{0}^{p_{r}} \int_{0}^{x} w_{0 r}(y) d y d x .
\end{aligned}
$$

Now we prove that this limit is equal to

$$
X_{\infty, 2}^{0}=-\min _{x \in \mathbb{R}} \int_{0}^{x} w_{0 l}(y) d y+\min _{x \in \mathbb{R}} \int_{0}^{x} w_{0 r}(y) d y .
$$

In fact, for $i=l$ or $r$, since the anti-derivative variable $\int_{0}^{x} w_{0 i}(y) d y$ is continuous and periodic with the period $p_{i}$, one can choose a constant $x_{i} \in\left[0, p_{i}\right)$ such that

$$
\int_{0}^{x_{i}} w_{0 i}(y) d y=\min _{x \in\left[0, p_{i}\right]} \int_{0}^{x} w_{0 i}(y) d y
$$

which is equivalent to

$$
\int_{x_{i}}^{x} w_{0 i}(y) d y \geqslant 0, \quad x \in \mathbb{R} .
$$

Then one can finish the proof of (1.12) if it holds that

$$
\int_{0}^{+\infty} \int_{0}^{p_{i}}\left(f\left(u_{i}^{0}\right)-f\left(\bar{u}_{i}\right)\right) d x d t=\int_{0}^{p_{i}} \int_{x_{i}}^{x} w_{0 i}(y) d y d x
$$

To prove (3.96), it follows from [2, Theorem 14.1.1] that the periodic entropy solution $u_{i}^{0}$ takes the constant value $\bar{u}_{i}$ along the straight line $x=x_{i}+f^{\prime}\left(\bar{u}_{i}\right) t$. Then for any given $y \in\left(x_{i}, x_{i}+p_{i}\right)$ and $t>0$, denote the domain:

$$
\Omega_{(y, t)}:=\left\{(x, \tau): x_{i}+f^{\prime}\left(\bar{u}_{i}\right) \tau<x<y+f^{\prime}\left(\bar{u}_{i}\right) \tau, 0<\tau<t\right\} .
$$

Integrating the equation $\partial_{t} u_{i}^{0}+\partial_{x} f\left(u_{i}^{0}\right)=0$ over $\Omega_{(y, t)}$, one can obtain that

$$
\begin{aligned}
0= & -\int_{x_{i}}^{y}\left(\bar{u}_{i}+w_{0 i}(x)\right) d x+\int_{0}^{t}\left(f\left(u_{i}^{0}\right)-f^{\prime}\left(\bar{u}_{i}\right) u_{i}^{0}\right)\left(y+f^{\prime}\left(\bar{u}_{i}\right) \tau, \tau\right) d \tau \\
& -\int_{0}^{t}\left(f\left(\bar{u}_{i}\right)-f^{\prime}\left(\bar{u}_{i}\right) \bar{u}_{i}\right) d \tau+\int_{x_{i}+f^{\prime}\left(\bar{u}_{i}\right) t}^{y+f^{\prime}\left(\bar{u}_{i}\right) t} u_{i}^{0}(x, t) d x \\
= & -\int_{x_{i}}^{y} w_{0 i}(x) d x+\int_{0}^{t}\left(f\left(u_{i}^{0}\right)-f\left(\bar{u}_{i}\right)\right)\left(y+f^{\prime}\left(\bar{u}_{i}\right) \tau, \tau\right) d \tau \\
& -f^{\prime}\left(\bar{u}_{i}\right) \int_{0}^{t}\left(u_{i}^{0}-\bar{u}_{i}\right)\left(y+f^{\prime}\left(\bar{u}_{i}\right) \tau, \tau\right) d \tau+\int_{x_{i}+f^{\prime}\left(\bar{u}_{i}\right) t}^{y+f^{\prime}\left(\bar{u}_{i}\right) t}\left(u_{i}^{0}(x, t)-\bar{u}_{i}\right) d x .
\end{aligned}
$$

Since for any $y \in \mathbb{R}, t \geqslant 0, \int_{y}^{y+p_{i}}\left(u_{i}^{0}-\bar{u}_{i}\right)(x, t) d x=0$, thus one can integrate (3.97) with respect to $y$ over $\left(x_{i}, x_{i}+p_{i}\right)$ to get that

$$
\begin{aligned}
0= & -\int_{x_{i}}^{x_{i}+p_{i}} \int_{x_{i}}^{y} w_{0 i}(x) d x d y+\int_{x_{i}}^{x_{i}+p_{i}} \int_{0}^{t}\left(f\left(u_{i}^{0}\right)-f\left(\bar{u}_{i}\right)\right)\left(y+f^{\prime}\left(\bar{u}_{i}\right) \tau, \tau\right) d \tau d y \\
& +\int_{x_{i}}^{x_{i}+p_{i}} \int_{x_{i}+f^{\prime}\left(\bar{u}_{i}\right) t}^{y+f^{\prime}\left(\bar{u}_{i}\right) t}\left(u_{i}^{0}(x, t)-\bar{u}_{i}\right) d x d y
\end{aligned}
$$


Since $\int_{x_{i}}^{y} w_{0 i}(x) d x$ is periodic with respect to $y$, and

$$
\int_{x_{i}}^{x_{i}+p_{i}} \int_{x_{i}+f^{\prime}\left(\bar{u}_{i}\right) t}^{y+f^{\prime}\left(\bar{u}_{i}\right) t}\left(u_{i}^{0}(x, t)-\bar{u}_{i}\right) d x d y=O\left(\frac{1}{1+t}\right),
$$

then it holds that

$$
\int_{0}^{t} \int_{0}^{p_{i}}\left(f\left(u_{i}^{0}(x, \tau)\right)-f\left(\bar{u}_{i}\right)\right) d x d \tau=\int_{0}^{p_{i}} \int_{x_{i}}^{y} w_{0 i}(x) d x d y+O\left(\frac{1}{1+t}\right) .
$$

Similar to the proof of (3.92), one can show that

$$
\int_{0}^{p_{i}}\left(f\left(u_{i}^{0}(x, \tau)\right)-f\left(\bar{u}_{i}\right)\right) d x=O\left(\frac{1}{(1+\tau)^{2}}\right) .
$$

Then (3.96) follows by letting $t \rightarrow+\infty$ in (3.98) and using the dominated convergence theorem.

It remains to prove (1.13) to finish the proof of Theorem 1.3. If both the periodic perturbations $w_{0 l}$ and $w_{0 r}$ have bounded total variations on the respective periodic domains:

$$
\mathrm{TV}_{\left[0, p_{l}\right]} w_{0 l}<+\infty, \quad \mathrm{TV}_{\left[0, p_{r}\right]} w_{0 r}<+\infty,
$$

then it can be derived from Kruzhkov's theory (see [21, 19]) that, for $i=l$ or $r$, the viscous solution $u_{i}^{\nu}$ to (1.1) tends to the inviscid entropy solution $u_{i}^{0}$ in the $L^{1}$ norm at the following rate:

$$
\int_{0}^{p_{i}}\left|u_{i}^{\nu}(x, t)-u_{i}^{0}(x, t)\right| d x \leqslant C(\nu t)^{1 / 2} \mathrm{TV}_{\left[0, p_{i}\right]} w_{0 i}, \quad t>0,0 \leqslant \nu \leqslant 1,
$$

where $C>0$ is independent of $\nu$ or $t$.

Then for any given $T>0$, and $i=l$ or $r$, one has that

$$
\begin{aligned}
& \int_{0}^{+\infty} \int_{0}^{p_{i}}\left(f\left(u_{i}^{\nu}\right)-f\left(\bar{u}_{i}\right)\right) d x d \tau-\int_{0}^{+\infty} \int_{0}^{p_{i}}\left(f\left(u_{i}^{0}\right)-f\left(\bar{u}_{i}\right)\right) d x d \tau \\
= & \int_{0}^{T} \int_{0}^{p_{i}}\left(f\left(u_{i}^{\nu}\right)-f\left(u_{i}^{0}\right)\right) d x d \tau+\int_{T}^{+\infty} \int_{0}^{p_{i}}\left(f\left(u_{i}^{\nu}\right)-f\left(\bar{u}_{i}\right)\right) d x d \tau \\
& -\int_{T}^{+\infty} \int_{0}^{p_{i}}\left(f\left(u_{i}^{0}\right)-f\left(\bar{u}_{i}\right)\right) d x d \tau .
\end{aligned}
$$

Then it follows from (3.92), (3.93) and (3.99) that for $i=l$ or $r$,

$$
\begin{aligned}
& \left|\int_{0}^{+\infty} \int_{0}^{p_{i}}\left(f\left(u_{i}^{\nu}\right)-f\left(\bar{u}_{i}\right)\right) d x d \tau-\int_{0}^{+\infty} \int_{0}^{p_{i}}\left(f\left(u_{i}^{0}\right)-f\left(\bar{u}_{i}\right)\right) d x d \tau\right| \\
& \leqslant C\left\{\nu^{1 / 2} \int_{0}^{T} t^{1 / 2} d t+\int_{T}^{+\infty} \frac{1}{(1+t)^{2}} d t\right\} \leqslant C\left(\nu^{1 / 2} T^{3 / 2}+T^{-1}\right) .
\end{aligned}
$$

Letting $T=\nu^{-1 / 5}$ in the above inequality yields that for $i=l$ or $r$,

$$
\left|\int_{0}^{+\infty} \int_{0}^{p_{i}}\left(f\left(u_{i}^{\nu}\right)-f\left(\bar{u}_{i}\right)\right) d x d \tau-\int_{0}^{+\infty} \int_{0}^{p_{i}}\left(f\left(u_{i}^{0}\right)-f\left(\bar{u}_{i}\right)\right) d x d \tau\right| \leqslant C \nu^{1 / 5} .
$$

It follows from this and the formulas of $X_{\infty, 2}^{\nu}$ and $X_{\infty, 2}^{0}$ that

$$
\left|X_{\infty, 2}^{\nu}-X_{\infty, 2}^{0}\right| \leqslant C \nu^{1 / 5},
$$

where $C>0$ is independent of $\nu$. 


\section{Stability of RAREFACTion WAVES}

The proof of Theorem 1.4 can follow from the idea in [16]. To make this paper complete, we still give the details here. The proof consists of two steps. The first step is to prove a time-independent estimate of the solution $u$, just as Proposition 3.4 for the shock profile. Step 2 is to construct an auxiliary function and use the maximal principle to complete the proof.

Proposition 4.1. For any $\varepsilon>0$, there exist $N_{\varepsilon}>0$ and $T_{\varepsilon}>0$ such that

$$
\begin{aligned}
& \left|u^{\nu}(x, t)-\bar{u}_{l}\right| \leqslant \varepsilon, \quad t>T_{\varepsilon}, x-f^{\prime}\left(\bar{u}_{l}\right) t<-N_{\varepsilon}, \\
& \left|u^{\nu}(x, t)-\bar{u}_{r}\right| \leqslant \varepsilon, \quad t>T_{\varepsilon}, x-f^{\prime}\left(\bar{u}_{r}\right) t>N_{\varepsilon} .
\end{aligned}
$$

Proof. We prove only (4.1), since the proof of (4.2) is similar.

For any $\varepsilon>0$, there exists $T>0$ such that $\left|u_{l}^{\nu}-\bar{u}_{l}\right|<\varepsilon \nu / 2$, for any $x \in \mathbb{R}, t \geqslant T$. Since $f$ is strictly convex, there exists $B>0$ such that, for any $0<\sigma<2\left\|u_{0}\right\|_{L^{\infty}(\mathbb{R})}$,

$$
f^{\prime}\left(\bar{u}_{l}+\sigma\right)-f^{\prime}\left(\bar{u}_{l}\right)>B \sigma .
$$

Without loss of generality, one can assume that $\varepsilon>0$ is small enough such that $B \varepsilon<2 \beta$, where $\beta>0$ is the constant in Lemma 2.3.

(1) By Lemma 2.3 and that $\left\|u^{\nu}\right\|_{L^{\infty}(d x, d t)} \leqslant\left\|u_{0}\right\|_{L^{\infty}}$, one can choose $M>0$ large enough such that

$$
M e^{\frac{B \varepsilon}{2}\left(x-f^{\prime}\left(\bar{u}_{l}\right) T\right)}+\frac{\varepsilon \nu}{2}+\bar{u}_{l}>u^{\nu}(x, T), \quad x \in \mathbb{R} .
$$

Define

$$
V(x, t):=M e^{\frac{B \varepsilon}{2}\left(x-f^{\prime}\left(\bar{u}_{l}\right) t\right)}+\frac{\varepsilon \nu}{2}+\bar{u}_{l}-u^{\nu}(x, t) .
$$

It can be checked easily that $V(x, T)>0$, and

$$
\nu \partial_{x}^{2} V-\partial_{t} V-f^{\prime}\left(u^{\nu}\right) \partial_{x} V=M \frac{B \varepsilon}{2} e^{\frac{B \varepsilon}{2}\left(x-f^{\prime}\left(\bar{u}_{l}\right) t\right)}\left(\frac{B \varepsilon \nu}{2}+f^{\prime}\left(\bar{u}_{l}\right)-f^{\prime}\left(u^{\nu}\right)\right) .
$$

If

$$
\min _{x \in \mathbb{R}, t \geqslant T} V(x, t)=V\left(x_{0}, t_{0}\right)<0,
$$

by the definition of $V$, one has that $\bar{u}_{l}-u^{\nu}\left(x_{0}, t_{0}\right)<-\frac{\varepsilon \nu}{2}$, and then

$$
f^{\prime}\left(\bar{u}_{l}\right)-f^{\prime}\left(u^{\nu}\left(x_{0}, t_{0}\right)\right)<f^{\prime}\left(\bar{u}_{l}\right)-f^{\prime}\left(\bar{u}_{l}+\frac{\varepsilon \nu}{2}\right) \leqslant-\frac{B \varepsilon \nu}{2} .
$$

Thus (4.3) yields that at the point $\left(x_{0}, t_{0}\right)$,

$$
\nu \partial_{x}^{2} V-\partial_{t} V-f^{\prime}\left(u^{\nu}\right) \partial_{x} V<0
$$

Therefore, it follows from the maximum principle ([16, Lemma 1]) that $V(x, t) \geqslant 0$ for any $x \in \mathbb{R}, t \geqslant T$. Choosing $N:=\frac{2}{B \varepsilon} \ln \frac{\varepsilon}{2 M}$, one has that for any $t>T$ and $x-f^{\prime}\left(\bar{u}_{l}\right) t<-N$, it holds that

$$
u^{\nu}(x, t) \leqslant \bar{u}_{l}+\frac{\varepsilon \nu}{2}+M e^{\frac{B \varepsilon}{2}\left(x-f^{\prime}\left(\bar{u}_{l}\right) t\right)}<\bar{u}_{l}+\varepsilon .
$$

(2) On the other hand, for the initial data (1.3) and any $\varepsilon>0$, one can let $\tilde{u}(x, t)$ be the unique solution to (1.1) with the $L^{\infty}$ initial data

$$
\tilde{u}(x, 0)= \begin{cases}u_{0}(x), & x<0, \\ \bar{u}_{l}-\varepsilon / 2+\left(u_{0}(x)-\bar{u}_{r}\right), & x>0,\end{cases}
$$


which satisfies

$$
\left\{\begin{array}{l}
\left|\tilde{u}(x, 0)-\bar{u}_{l}-w_{0 l}(x)\right| \leqslant C_{0} e^{\beta_{0} x}, \\
\left|\tilde{u}(x, 0)-\left(\bar{u}_{l}-\varepsilon / 2\right)-w_{0 r}(x)\right| \leqslant C_{0} e^{-\beta_{0} x}, \quad x \in \mathbb{R} .
\end{array}\right.
$$

Theorem 1.1 implies that as $t \rightarrow+\infty, \tilde{u}$ tends to a viscous shock profile $\tilde{\phi}$ connecting $\bar{u}_{l}$ as $x \rightarrow-\infty$ and $\bar{u}_{l}-\varepsilon / 2$ as $x \rightarrow+\infty$. Thus, there exists $T>0$ such that for any $x \in \mathbb{R}, t>T$,

$$
\tilde{u}(x, t) \geqslant \tilde{\phi}(x-s t)-\varepsilon / 2 \geqslant \bar{u}_{l}-\varepsilon .
$$

By $\tilde{u}(x, 0) \leqslant u_{0}(x)$ and the comparison principle, one has

$$
u^{\nu}(x, t) \geqslant \tilde{u}(x, t) \geqslant \bar{u}_{l}-\varepsilon, \quad x \in \mathbb{R}, t \geqslant 0 .
$$

Then (4.1) follows from (4.4) and (4.6).

Proof of Theorem 1.4. It is equivalent to prove that for any $\varepsilon>0$, there exists $T>0$ such that

$$
\sup _{x \in \mathbb{R}}\left|u^{\nu}(x, t)-u^{R}(x, t)\right|<\varepsilon, \quad t>T .
$$

For the constants $N_{\varepsilon}$ and $T_{\varepsilon}$ in Proposition 4.1, one can define two constants

$$
x_{0}:=N_{\varepsilon} \frac{f^{\prime}\left(\bar{u}_{l}\right)+f^{\prime}\left(\bar{u}_{r}\right)}{f^{\prime}\left(\bar{u}_{l}\right)-f^{\prime}\left(\bar{u}_{r}\right)} \text { and } t_{0}:=\frac{-2 N_{\varepsilon}}{f^{\prime}\left(\bar{u}_{l}\right)-f^{\prime}\left(\bar{u}_{r}\right)}>0,
$$

and the region

$$
\Omega_{\varepsilon}:=\left\{(x, t): f^{\prime}\left(\bar{u}_{l}\right) t-N_{\varepsilon}<x<f^{\prime}\left(\bar{u}_{r}\right) t+N_{\varepsilon}, t>T_{\varepsilon}\right\} .
$$

Then the shifted rarefaction wave $\tilde{u}^{R}(x, t):=u^{R}\left(x-x_{0}, t+t_{0}\right)$ satisfies that

$$
\tilde{u}^{R}(x, t)= \begin{cases}\bar{u}_{l} & \text { for } x-f^{\prime}\left(\bar{u}_{l}\right) t \leqslant-N_{\varepsilon}, \\ \left(f^{\prime}\right)^{-1}\left(\frac{x-x_{0}}{t+t_{0}}\right) & \text { for } x \in \Omega_{\varepsilon} \\ \bar{u}_{r} & \text { for } x-f^{\prime}\left(\bar{u}_{r}\right) t \geqslant N_{\varepsilon} .\end{cases}
$$

Therefore, Proposition 4.1 implies that for any $x<f^{\prime}\left(\bar{u}_{l}\right) t-N_{\varepsilon}$ or $x>f^{\prime}\left(\bar{u}_{r}\right) t+$ $N_{\varepsilon}, t \geqslant T_{\varepsilon}$, one has that

$$
\left|u^{\nu}(x, t)-\tilde{u}^{R}(x, t)\right|<\varepsilon .
$$

Define

$$
Z(x, t):=\left(t+t_{0}\right)^{\kappa}\left(u^{\nu}(x, t)-\tilde{u}^{R}(x, t)\right),
$$

where $0<\kappa<1$ is a constant to be determined. Direct calculations show that

$$
\nu \partial_{x}^{2} Z-f^{\prime}\left(u^{\nu}\right) \partial_{x} Z-\partial_{t} Z=\left(f^{\prime \prime}(v) \partial_{x} \tilde{u}^{R}-\frac{\kappa}{t+t_{0}}\right) Z-\nu\left(t+t_{0}\right)^{\kappa} \partial_{x}^{2} \tilde{u}^{R} \quad \text { in } \Omega_{\varepsilon}
$$

where $v$ is the function satisfying

$$
f^{\prime}\left(u^{\nu}\right)-f^{\prime}\left(\tilde{u}^{R}\right)=f^{\prime \prime}(v)\left(u^{\nu}-\tilde{u}^{R}\right) .
$$

For $(x, t) \in \Omega_{\varepsilon}$, it holds that $f^{\prime \prime}(v) \partial_{x} \tilde{u}^{R}=f^{\prime \prime}(v) \frac{1}{f^{\prime \prime}\left(\tilde{u}^{R}\right)} \frac{1}{t+t_{0}}>\frac{2 \omega}{t+t_{0}}$ for some $\omega>0$. Then $0<\kappa<1$ can be chosen small enough such that

$$
f^{\prime \prime}(v) \partial_{x} \tilde{u}^{R}-\frac{\kappa}{t+t_{0}}>\frac{\omega}{t+t_{0}}>0 .
$$


Note that for any $x \in \mathbb{R}$,

$$
\left|Z\left(x, T_{\varepsilon}\right)\right| \leqslant M_{4}\left(T_{\varepsilon}+t_{0}\right)^{\kappa},
$$

provided that $M_{4}>\left\|u_{0}\right\|_{L^{\infty}}+\left\|u^{R}\right\|_{L^{\infty}}$ is large enough. Due to (4.8), one has that for any $t \geqslant T_{\varepsilon}$,

$$
|Z(x, t)| \leqslant \varepsilon\left(t+t_{0}\right)^{\kappa}
$$

if $x=f^{\prime}\left(\bar{u}_{l}\right) t-N_{\varepsilon}$ or $f^{\prime}\left(\bar{u}_{r}\right) t+N_{\varepsilon}$. Now, assume that the maximum value $\max _{\Omega_{\varepsilon}} Z$ is achieved at $\left(x_{1}, t_{1}\right) \in \Omega_{\varepsilon}$ and satisfies that

$$
Z\left(x_{1}, t_{1}\right) \geqslant \max \left\{M_{4}\left(T_{\varepsilon}+t_{0}\right)^{\kappa}, \varepsilon\left(t_{1}+t_{0}\right)^{\kappa}, \frac{M_{4}}{\omega}\left(T_{\varepsilon}+t_{0}\right)^{\kappa-1}\right\}>0 .
$$

Then by (4.10)-(4.12), one has that at $\left(x_{1}, t_{1}\right)$,

$$
\nu \partial_{x}^{2} Z-f^{\prime}\left(u^{\nu}\right) \partial_{x} Z-\partial_{t} Z \geqslant \frac{\omega}{t_{1}+t_{0}} \frac{M_{4}}{\omega}\left(T_{\varepsilon}+t_{0}\right)^{\kappa-1}-\nu\left(t_{1}+t_{0}\right)^{\kappa} \frac{M_{5}}{\left(t_{1}+t_{0}\right)^{2}}>0,
$$

where $M_{5}=\max \left|\left(\left(f^{\prime}\right)^{-1}\right)^{\prime \prime}\right|$ and $M_{4}>M_{5}$ is large enough. Therefore, the maximal principle implies that for any $(x, t) \in \Omega_{\varepsilon}$,

$$
Z(x, t) \leqslant \max \left\{M_{4}\left(T_{\varepsilon}+t_{0}\right)^{\kappa}, \varepsilon\left(t+t_{0}\right)^{\kappa}, \frac{M_{4}}{\omega}\left(T_{\varepsilon}+t_{0}\right)^{\kappa-1}\right\} .
$$

Similarly, one can verify that for any $(x, t) \in \Omega_{\varepsilon}$,

$$
Z(x, t) \geqslant \min \left\{-M_{4}\left(T_{\varepsilon}+t_{0}\right)^{\kappa},-\varepsilon\left(t+t_{0}\right)^{\kappa},-\frac{M_{4}}{\omega}\left(T_{\varepsilon}+t_{0}\right)^{\kappa-1}\right\} .
$$

As a result, by choosing a large $T>T_{\varepsilon}$, one can get that

$$
\left|u^{\nu}(x, t)-u^{R}\left(x-x_{0}, t+t_{0}\right)\right| \leqslant \max \left\{\frac{M_{4}\left(T_{\varepsilon}+t_{0}\right)^{\kappa}}{\left(t+t_{0}\right)^{\kappa}}, \varepsilon, \frac{M_{4}}{\left(T_{\varepsilon}+t_{0}\right)^{1-\kappa}\left(t+t_{0}\right)^{\kappa}}\right\} \leqslant \varepsilon
$$

for any $f^{\prime}\left(\bar{u}_{l}\right) t-N_{\varepsilon}<x<f^{\prime}\left(\bar{u}_{r}\right) t+N_{\varepsilon}$ and $t>T$. Moreover, since $u^{R}$ is Lipschitz continuous and $\frac{x}{t}-\frac{x-x_{0}}{t+t_{0}} \rightarrow 0$ as $t \rightarrow \infty$, (4.7) follows easily from combining Proposition 4.1 and (4.13), and thus Theorem 1.4 is proved.

\section{Appendix A. Proof of Lemma 2.1}

Proof. For convenience, we let $\nu=1$ and omit the symbol $\nu$. By multiplying $u-\bar{u}$ on each side of (1.1) and integrating on $[0, p]$, it holds that

$$
\frac{d}{d t} \int_{0}^{p}(u-\bar{u})^{2}(x, t) d x+2 \int_{0}^{p}\left(\partial_{x} u\right)^{2}(x, t) d x=0, \quad t>0 .
$$

By the Poincaré inequality on $[0, p]$, there exists a constant $\alpha>0$, which depends only on $p$, such that

$$
\int_{0}^{p}\left(\partial_{x} u\right)^{2}(x, t) d x \geqslant \frac{\alpha}{2} \int_{0}^{p}(u-\bar{u})^{2}(x, t) d x .
$$

Then by (A.1), one has

$$
\int_{0}^{p}(u-\bar{u})^{2}(x, t) d x \leqslant C_{0} e^{-\alpha t} \quad \forall t \geqslant 0,
$$


where $C_{0}=\int_{0}^{p}\left(u_{0}-\bar{u}\right)^{2} d x$ depends on $p$ and $\left\|u_{0}\right\|_{L^{\infty}}$.

Claim 1. For any integer $k \geqslant 1$,

$$
\int_{0}^{p}\left(\partial_{x}^{k} u\right)^{2}(x, t) d x \leqslant C \quad \forall t \geqslant 1,
$$

where $C$ depends on $k, p, f$ and $\left\|u_{0}\right\|_{L^{\infty}}$.

In fact, for each $k \geqslant 0$, we let $t_{k}:=\frac{1}{2}-\frac{1}{k+3}$, and define smooth functions $\zeta_{k}(t)$ : $[0,+\infty) \rightarrow[0,1]$, which are non-decreasing and satisfy that for all $t \geqslant 0$,

$$
\zeta_{k}(t)=\left\{\begin{array}{ll}
0, & t \in\left[0, t_{k}\right] \\
1, & t \in[1,+\infty)
\end{array} \quad \text { and } \quad \zeta_{k}^{\prime}(t)+\zeta_{k}(t) \leqslant B_{k} \zeta_{k-1}(t)\right.
$$

where $B_{k}>0$ is a constant depending on $k$; see Figure 3 .

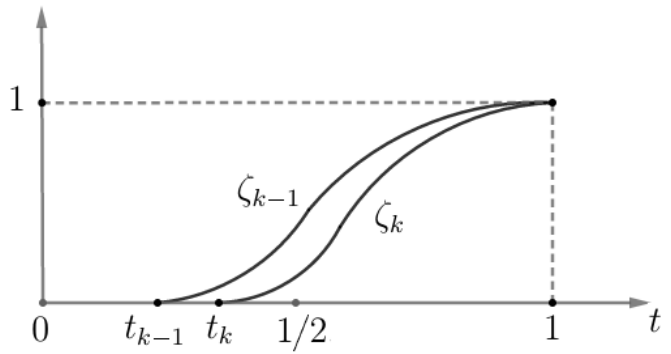

FIGURE 3.

Then we prove Claim 1 by the induction method. We will prove that for each $k \geqslant 1$, there exists a constant $C>0$, depending on $k, p, f$ and $\left\|u_{0}\right\|_{L^{\infty}}$, such that

$$
\int_{0}^{p}\left(\partial_{x}^{k-1}(u-\bar{u})\right)^{2} d x+\int_{0}^{t} \zeta_{k-1}(\tau) \int_{0}^{p}\left(\partial_{x}^{k} u\right)^{2} d x d \tau \leqslant C \quad \forall t>t_{k} .
$$

In fact, when $k=1$, (A.3) follows from (A.1). Then we assume that (A.3) holds for $k=1,2, \cdots, m$ with $m \geqslant 1$, and then we will prove that (A.3) also holds for $k=m+1$. By taking the derivative $\partial_{x}^{m}$ in (1.1) and multiplying $\zeta_{m} \partial_{x}^{m} u$ on each side, one can obtain

$$
\begin{aligned}
& \partial_{t}\left(\zeta_{m}\left(\partial_{x}^{m} u\right)^{2}\right)-\zeta_{m}^{\prime}\left(\partial_{x}^{m} u\right)^{2}+\partial_{x}\left(\zeta_{m} \partial_{x}^{m} u \partial_{x}^{m} f(u)\right)-\zeta_{m} \partial_{x}^{m+1} u \partial_{x}^{m} f(u) \\
= & \partial_{x}\left(\zeta_{m} \partial_{x}^{m} u \partial_{x}^{m+1} u\right)-\zeta_{m}\left(\partial_{x}^{m+1} u\right)^{2} .
\end{aligned}
$$

This, with the Cauchy-Schwartz inequality, yields that for all $t>0$,

$$
\begin{aligned}
& \frac{d}{d t} \int_{0}^{p} \zeta_{m}(t)\left(\partial_{x}^{m} u\right)^{2} d x+\zeta_{m}(t) \int_{0}^{p}\left(\partial_{x}^{m+1} u\right)^{2} d x \\
\leqslant & C\left(\zeta_{m}^{\prime}+\zeta_{m}\right) \sum_{k=1}^{m} \int_{0}^{p}\left(\partial_{x}^{k} u\right)^{2} d x \\
\leqslant & C B_{m} \zeta_{m-1}(t) \sum_{k=1}^{m} \int_{0}^{p}\left(\partial_{x}^{k} u\right)^{2} d x .
\end{aligned}
$$


Thus one can have that for all $t>0$,

$$
\begin{aligned}
& \int_{0}^{p} \zeta_{m}(t)\left(\partial_{x}^{m} u\right)^{2} d x+\int_{0}^{t} \zeta_{m}(\tau) \int_{0}^{p}\left(\partial_{x}^{m+1} u\right)^{2} d x d \tau \\
\leqslant & C_{m} \sum_{k=1}^{m} \int_{0}^{t} \zeta_{k-1}(\tau) \int_{0}^{p}\left(\partial_{x}^{k} u\right)^{2} d x d \tau
\end{aligned}
$$

where $C_{m}>0$ depends on $f, p$ and $\left\|u_{0}\right\|_{L^{\infty}}$. Then by (A.3) for $k=1,2, \cdots, m$, for any $t>t_{m}$, the right hand side of (A.5) is bounded by a constant, so (A.3) holds true for $k=m+1$. Thus, by the induction method, (A.3) holds true for any $k \geqslant 1$ and any $t \geqslant 1$, which completes the proof of Claim 1 .

Then it follows from Sobolev inequality, Claim 1, and the equation (1.1) that for any integers $k, l \geqslant 0$,

$$
\left\|\partial_{t}^{l} \partial_{x}^{k}(u-\bar{u})\right\|_{L^{\infty}(\mathbb{R})} \leqslant C_{k l} \quad \forall t \geqslant 1 .
$$

And since for each $k \geqslant 0, \zeta_{k}(t)=1$ and $\zeta_{k}^{\prime}(t)=0$ for all $t \geqslant 1,($ A.1) and (A.4) yield that

$$
\frac{d}{d t} \int_{0}^{p}\left(\partial_{x}^{k}(u-\bar{u})\right)^{2} d x+\int_{0}^{p}\left(\partial_{x}^{k+1} u\right)^{2} d x \leqslant C \sum_{l=1}^{k} \int_{0}^{p}\left(\partial_{x}^{l} u\right)^{2} d x \quad \forall t \geqslant 1,
$$

where $C>0$ depends on $k, p, f$ and $\left\|u_{0}\right\|_{L^{\infty}}$.

Claim 2. For each $k \geqslant 0$, there holds that

$$
\int_{0}^{p}\left(\partial_{x}^{k}(u-\bar{u})\right)^{2}(x, t) d x \leqslant C e^{-\alpha t} \quad \forall t \geqslant 1
$$

where $C>0$ depends on $k, p, f$ and $\left\|u_{0}\right\|_{L^{\infty}}$.

To prove Claim 2, we also use the induction method. For $k=0$, (A.7) follows from (A.2). Thus, one can assume that for $k=0,1, \cdots, m-1$ with $m \geqslant 1$, Claim 2 is true. Then for $k=m$, by (A.6) with $k=m$, one has that for all $t \geqslant 1$,

$$
\begin{aligned}
\frac{d}{d t} \int_{0}^{p}\left(\partial_{x}^{m} u\right)^{2} d x & \leqslant C_{m} \sum_{k=1}^{m} \int_{0}^{p}\left(\partial_{x}^{k} u\right)^{2} d x \\
& \leqslant C e^{-\alpha t}+C_{m} \int_{0}^{p}\left(\partial_{x}^{m} u\right)^{2} d x
\end{aligned}
$$

where $C, C_{m}>0$ depend on $m, p, f$ and $\left\|u_{0}\right\|_{L^{\infty}}$, and $C_{m}$ can be large enough such that $C_{m}>\alpha$. Letting $k=m-1$ in (A.6), one gets that for all $t \geqslant 1$,

$$
\frac{d}{d t} \int_{0}^{p}\left(\partial_{x}^{m-1}(u-\bar{u})\right)^{2} d x+\int_{0}^{p}\left(\partial_{x}^{m} u\right)^{2} d x \leqslant C e^{-\alpha t} .
$$

Then by multiplying $2 C_{m}$ on (A.9) and then adding it to (A.8), one can obtain that for all $t \geqslant 1$,

$$
\frac{d}{d t}\left[2 C_{m} \int_{0}^{p}\left(\partial_{x}^{m-1}(u-\bar{u})\right)^{2} d x+\int_{0}^{p}\left(\partial_{x}^{m} u\right)^{2} d x\right]+C_{m} \int_{0}^{p}\left(\partial_{x}^{m} u\right)^{2} d x \leqslant C e^{-\alpha t} .
$$

Denote

$$
E_{m}(t):=2 C_{m} \int_{0}^{p}\left(\partial_{x}^{m-1}(u-\bar{u})\right)^{2} d x+\int_{0}^{p}\left(\partial_{x}^{m} u\right)^{2} d x
$$


Then (A.7) with $k=m-1$ and (A.10) yield that for all $t \geqslant 1$,

$$
E_{m}^{\prime}(t)+C_{m} E_{m}(t) \leqslant C_{m}^{\prime} e^{-\alpha t} .
$$

Since $C_{m}>\alpha$, one can easily obtain that $E_{m}(t) \leqslant C e^{-\alpha t}$, where $C>0$ depends on depends on $m, p, f$ and $\left\|u_{0}\right\|_{L^{\infty}}$. The proof of Claim 2 is finished.

Then by Sobolev inequality and Claim 2, and combined with the equation (1.1), one can have that for any integers $k, l \geqslant 0$ and $t \geqslant 1$,

$$
\left\|\partial_{t}^{l} \partial_{x}^{k}(u-\bar{u})\right\|_{L^{\infty}(\mathbb{R})} \leqslant C e^{-\alpha t},
$$

which finishes the proof of Lemma 2.1.

\section{Appendix B. Proof of Lemma 2.2}

Proof. For convenience, we assume that $\nu=1$ and omit the symbol $\nu$. And let $K^{t}(x):=\frac{1}{\sqrt{2 \pi t}} e^{-\frac{x^{2}}{4 t}}$ denote the heat kernel.

As in [5], the solution $S_{t} u_{0}$ can be obtained by constructing the following approximating sequence

$$
\begin{aligned}
u^{(1)} & =K^{t} * u_{0}, \\
u^{(n+1)} & =K^{t} * u_{0}-\int_{0}^{t} \partial_{x} K^{\tau}(\cdot) * f\left(u^{(n)}(\cdot, t-\tau)\right) d \tau, \quad n=1,2,3, \ldots
\end{aligned}
$$

where "*" represents the convolution operation with respect to the space variable.

Suppose that $\left\{\tilde{u}^{(n)}\right\}_{n=1}^{\infty}$ is the approximating sequence induced by $\tilde{u}_{0}$, constructed in the same way as $u^{(n)}$. Therefore, one has that

$$
\begin{aligned}
\left|u^{(1)}-\tilde{u}^{(1)}\right| & =\left|K^{t} *\left(u_{0}-v_{0}\right)\right| \leqslant \int_{\mathbb{R}} \frac{1}{\sqrt{2 \pi t}} e^{-\frac{y^{2}}{4 t}} C e^{\delta(x-y)} d y \\
& \leqslant \int_{\mathbb{R}} \frac{1}{\sqrt{2 \pi t}} e^{-\frac{1}{4 t}(y+2 \delta t)^{2}} C e^{\delta x+\delta^{2} t} d y=C e^{\delta x+\delta^{2} t}, \\
\left|u^{(2)}-\tilde{u}^{(2)}\right| & \leqslant\left|K^{t} *\left(u_{0}-\tilde{u}_{0}\right)\right|+\mid \int_{0}^{t} \partial_{x} K^{\tau}(\cdot) *\left[f\left(u^{(1)}(\cdot, t-\tau)\right)\right. \\
& \left.\left.\leqslant C e^{\delta x+\delta^{2} t}+\int_{0}^{t} \int_{\mathbb{R}} \frac{1}{\sqrt{2 \pi \tau}} \frac{|y|}{2 \tau} e^{-\frac{y^{2}}{4 \tau}} C_{0} C e^{\delta(1)}(\cdot, t-\tau)\right)\right] d \tau \mid \\
& \leqslant C e^{\delta x+\delta^{2} t}+C_{0} C e^{\delta x+\delta^{2} t} \int_{0}^{t} \int_{\mathbb{R}} \frac{1}{\sqrt{2 \pi \tau}(t-\tau)} d y d \tau \mid \frac{|y|}{2 \tau} e^{-\frac{(y+2 \delta \tau)^{2}}{4 \tau}} d y d \tau \\
& \leqslant C e^{\delta x+\delta^{2} t}+C_{0} C e^{\delta x+\delta^{2} t} \int_{0}^{t} \int_{\mathbb{R}} \frac{1}{\sqrt{2 \pi \tau}}\left(\frac{|y|}{2 \tau}+|\delta|\right) e^{-\frac{y^{2}}{4 \tau}} d y d \tau \\
& =C e^{\delta x+\delta^{2} t}+C_{0} C e^{\delta x+\delta^{2} t} \int_{0}^{t}\left(\sqrt{\frac{2}{\pi \tau}}+|\delta|\right) d \tau \\
& \leqslant C e^{\delta x+\delta^{2} t}\left(1+\frac{2 \sqrt{2}}{\sqrt{\pi}} C_{0} \sqrt{t}+C_{0}|\delta| t\right)
\end{aligned}
$$


where $C_{0}:=\max \left\{\left|f^{\prime}(u)\right|:|u| \leqslant\left\|u_{0}, \tilde{u}_{0}\right\|_{L^{\infty}(\mathbb{R})}\right\}$. By induction, one has that for $t>0$ small,

$$
\begin{aligned}
\left|u^{(n)}-\tilde{u}^{(n)}\right| & \leqslant C e^{\delta x+\delta^{2} t}\left[1+\left(\frac{2 \sqrt{2}}{\sqrt{\pi}} C_{0} \sqrt{t}+C_{0}|\delta| t\right)+\cdots+\left(\frac{2 \sqrt{2}}{\sqrt{\pi}} C_{0} \sqrt{t}+C_{0}|\delta| t\right)^{n-1}\right] \\
& \leqslant C e^{\delta x+\delta^{2} t} \frac{1}{1-\left(\frac{2 \sqrt{2}}{\sqrt{\pi}} C_{0} \sqrt{t}+C_{0}|\delta| t\right)} .
\end{aligned}
$$

Therefore, by letting $n \rightarrow+\infty$, there exists a small enough $t_{0}=t_{0}\left(|\delta|, C_{0}\right)$ with

$$
e^{\delta^{2} t_{0}} \frac{1}{1-\left(\frac{2 \sqrt{2}}{\sqrt{\pi}} C_{0} \sqrt{t_{0}}+C_{0}|\delta| t_{0}\right)}<2,
$$

such that $\left|S_{t} u_{0}-S_{t} \tilde{u}_{0}\right| \leqslant 2 C e^{\delta x}$ holds for any $x \in \mathbb{R}, t \in\left(0, t_{0}\right]$. At time $t=k t_{0}$, $k=1,2,3, \ldots$, one can take $S_{k t_{0}} u_{0}, S_{k t_{0}} \tilde{u}_{0}$ instead of $u_{0}, \tilde{u}_{0}$ as the initial data and then repeat the same estimates as above in the interval $\left[k t_{0},(k+1) t_{0}\right]$. It concludes that for any $x \in \mathbb{R}, t>0,\left|S_{t} u_{0}-S_{t} \tilde{u}_{0}\right| \leqslant 2^{\frac{t}{t_{0}}+1} C e^{\delta x}$.

\section{Appendix C. Proof of Lemma 2.4}

Proof. For convenience, we let $\nu=1$ and omit the symbol $\nu$. Integrating the equation (1.5) shows that the shock profile $\phi$ satisfies

$$
\phi^{\prime}=f(\phi)-f\left(\bar{u}_{l}\right)-s\left(\phi-\bar{u}_{l}\right),
$$

which implies that

$$
\phi^{\prime}=\left(\phi-\bar{u}_{l}\right)\left(\frac{f(\phi)-f\left(\bar{u}_{l}\right)}{\phi-\bar{u}_{l}}-s\right)=\left(\phi-\bar{u}_{r}\right)\left(\frac{f(\phi)-f\left(\bar{u}_{r}\right)}{\phi-\bar{u}_{r}}-s\right) .
$$

thus one has that the function $g^{\nu}(x)$ defined in (2.3) satisfies the equation:

$$
\begin{aligned}
g^{\prime} & =\frac{1}{\bar{u}_{l}-\bar{u}_{r}}\left[f\left(\left(\bar{u}_{l}-\bar{u}_{r}\right) g+\bar{u}_{r}\right)-f\left(\bar{u}_{r}\right)\right]-s g \\
& =\frac{1}{\bar{u}_{l}-\bar{u}_{r}}\left[f\left(\bar{u}_{l} g+\bar{u}_{r}(1-g)\right)-f\left(\bar{u}_{l}\right) g-f\left(\bar{u}_{r}\right)(1-g)\right] .
\end{aligned}
$$

(i). Since $f$ is smooth, for any $x>y, 0 \leqslant \rho \leqslant 1, z=\rho x+(1-\rho) y$, one has

$$
\begin{aligned}
& f(z)-[\rho f(x)+(1-\rho) f(y)] \\
= & \rho(z-x) \int_{0}^{1} f^{\prime}(\tau z+(1-\tau) x) d \tau+(1-\rho)(z-y) \int_{0}^{1} f^{\prime}(\tau z+(1-\tau) y) d \tau \\
= & \rho(1-\rho)(x-y) \int_{0}^{1} \int_{0}^{1}(y-x)(1-\tau) \times \\
& f^{\prime \prime}(\tilde{\tau}[\tau z+(1-\tau) y]+(1-\tilde{\tau})[\tau z+(1-\tau) x]) d \tilde{\tau} d \tau .
\end{aligned}
$$

Therefore,

$$
\frac{1}{2} \min _{u \in[y, x]} f^{\prime \prime}(u) \leqslant-\frac{f(z)-[\rho f(x)+(1-\rho) f(y)]}{\rho(1-\rho)(x-y)^{2}} \leqslant \frac{1}{2} \max _{u \in[y, x]} f^{\prime \prime}(u) .
$$

Then (2.4) follows by substituting $x=\bar{u}_{l}, y=\bar{u}_{r}, \rho=g$ and $z=\bar{u}_{l} g+\bar{u}_{r}(1-g)$, and applying the definition (C.1). 
(ii). Integrating the equation (2.4) yields

$$
\begin{aligned}
& \beta_{1} x \leqslant \ln \frac{1-g(x)}{g(x)}-\beta_{3} \leqslant \beta_{2} x, \quad x>0, \\
& \beta_{2} x \leqslant \ln \frac{1-g(x)}{g(x)}-\beta_{3} \leqslant \beta_{1} x, \quad x<0,
\end{aligned}
$$

where $\beta_{3}=\ln \frac{1-g(0)}{g(0)}$. And then

$$
\begin{gathered}
\frac{1}{1+e^{\beta_{2} x+\beta_{3}}} \leqslant g(x) \leqslant \frac{1}{1+e^{\beta_{1} x+\beta_{3}}}, \quad x>0, \\
\frac{e^{\beta_{2} x+\beta_{3}}}{1+e^{\beta_{2} x+\beta_{3}}} \leqslant 1-g(x) \leqslant \frac{e^{\beta_{1} x+\beta_{3}}}{1+e^{\beta_{1} x+\beta_{3}}}, \quad x<0 .
\end{gathered}
$$

Therefore, (2.5) follows, and $C$ depends on $\beta_{1}, \beta_{2}$ and $\beta_{3}$.

\section{REFERENCES}

1. C. M. Dafermos, Large time behavior of periodic solutions of hyperbolic systems of conservation laws, Journal of Differential Equations 121 (1995), no. 1, 183-202.

2. __ Hyperbolic conservation laws in continuum physics, fourth ed., Grundlehren der Mathematischen Wissenschaften [Fundamental Principles of Mathematical Sciences], vol. 325, Springer-Verlag, Berlin, 2016. MR 3468916

3. A.-L. Dalibard, Long time behavior of parabolic scalar conservation laws with space periodic flux, Indiana Univ. Math. J. 59 (2010), no. 1, 257-300.

4. A. L. Dalibard and M. J. Kang, Existence and stability of planar shocks of viscous scalar conservation laws with space-periodic flux, Journal des Mathematiques Pures et Appliquees 107 (2017), no. 3, 336-366.

5. H. Freistühler and D. Serre, $l^{1}$ stability of shock waves in scalar viscous conservation laws, Communications on Pure and Applied Mathematics 51 (1998), no. 3, 291-301.

6. A. Friedman, Partial Differential Equations of Parabolic Type, Prentice-Hall, Inc., Englewood Cliffs, N.J., 1964.

7. J. Glimm and P. D. Lax, Decay of solutions of systems of nonlinear hyperbolic conservation laws, Memoirs of the American Mathematical Society, No. 101, American Mathematical Society, Providence, R.I., 1970.

8. J. Goodman, Nonlinear asymptotic stability of viscous shock profiles for conservation laws, Archive for Rational Mechanics and Analysis 95 (1986), no. 4, 325-344.

9. __ Stability of viscous scalar shock fronts in several dimensions, Trans. Amer. Math. Soc. 311 (1989), no. 2, 683-695.

10. J. Goodman and Z. Xin, Viscous limits for piecewise smooth solutions to systems of conservation laws, Archive for Rational Mechanics and Analysis 121 (1992), no. 3, 235-265.

11. E. Harabetian, Rarefactions and large time behavior for parabolic equations and monotone schemes, Communications in Mathematical Physics 114 (1988), no. 4, 527-536.

12. D. Hoff and K. Zumbrun, Asymptotic behavior of multidimensional scalar viscous shock fronts, Indiana University Mathematics Journal 49 (2000), no. 2, 427-474.

13. __ Pointwise green's function bounds for multidimensional scalar viscous shock fronts, J. Differential Equations 183 (2002), no. 2, 368-408.

14. E. Hopf, The partial differential equation $u_{t}+u u_{x}=\mu u_{x x}$, Communications on Pure and Applied Mathematics 3 (1950), no. 3, 201-230.

15. P. Howard, Pointwise green's function approach to stability for scalar conservation laws, Communications on Pure and Applied Mathematics 52 (1999), no. 10, 1295-1313. 
16. A. M. Il'in and O. A. Oleǐnik, Asymptotic behavior of solutions of the cauchy problem for some quasilinear equations for large values of time, Matematicheskii Sbornik 51(93) (1960), no. 2, 191-216.

17. C. E. Kenig and F. Merle, Asymptotic stability and liouville theorem for scalar viscous conservation laws in cylinders, Communications on Pure and Applied Mathematics 59 (2006), no. 6, 769-796.

18. N. Kopell and L. N. Howard, Bifurcations and trajectories joining critical points, Advances in Mathematics 18 (1975), no. 3, 306-358.

19. H. O. Kreiss, Fourier expansions of the solutions of the navier-stokes equations and their exponential decay rate, Analyse mathématique et applications, Gauthier-Villars, Montrouge, 1988, pp. 245-262.

20. S N Kruzkov, First order quasilinear equations with several independent variables, Mat. Sb. (N.S.) 81 (123) (1970), 228-255.

21. N. N. Kuznetsov, Accuracy of some approximate methods for computing the weak solutions of a first-order quasi-linear equation, USSR Computational Mathematics and Mathematical Physics 16 (1976), no. 6, 105-119.

22. P. D. Lax, Hyperbolic systems of conservation laws ii, Communications on Pure and Applied Mathematics 10 (1957), no. 4, 537-566.

23. T.-P. Liu, Nonlinear stability of shock waves for viscous conservation laws, Bullentin (New Series) of the American Mathematical Society 12 (1985), no. 2, 233-236.

24. __ Pointwise convergence to shock waves for viscous conservation laws, Communications on Pure and Applied Mathematics 50 (1997), no. 11, 1113-1182.

25. T.-P. Liu and Z. Xin, Nonlinear stability of rarefaction waves for compressible Navier-Stokes equations, Communications in Mathematical Physics 118 (1988), 451-465.

26. A. Matsumura and K. Nishihara, On the stability of travelling wave solutions of a onedimensional model system for compressible viscous gas, Japan J. Appl. Math. 2 (1985), no. 1, $17-25$.

27. K. Nishihara, A note on the stability of travelling wave solutions of burgers' equation, Japan Journal of Applied Mathematics 2 (1985), no. 1, 27-35.

28. O. A. Oleǐnik, Discontinuous solutions of non-linear differential equations, Uspehi Mat. Nauk (N.S.) 12 (1957), no. 3(75), 3-73.

29. A. Szepessy and Z. Xin, Nonlinear stability of viscous shock waves, Archive for Rational Mechanics and Analysis 122 (1993), no. 1, 53-103.

30. Z. Xin, Lectures on Hyperbolic Conservation Laws, IMS, CUHK.

31. Asymptotic stability of rarefaction waves for $2 \times 2$ viscous hyperbolic conservation laws, Journal of Differential Equations 73 (1988), no. 1, 45-77.

32. __ Asymptotic stability of planar rarefaction waves for viscous conservation laws in several dimensions, Trans. Amer. Math. Soc. 319 (1990), no. 2, 805-820.

33. Z. Xin, Q. Yuan, and Y. Yuan, Asymptotic stability of shock waves and rarefaction waves under periodic perturbations for 1-d convex scalar conservation laws, SIAM Journal on Mathematical Analysis 51 (2019), no. 4, 2971-2994.

34. Q. Yuan and Y. Yuan, On Riemann solutions under different initial periodic perturbations at two infinities for 1-d scalar convex conservation laws, arXiv: 1907.13043 (2019), 1-15.

(Z. Xin) The Institute of Mathematical Sciences \& Department of Mathematics, The Chinese University of Hong Kong, Shatin, N.T., Hong Kong

E-mail address: zpxin@ims.cuhk.edu.hk

(Q. Yuan) The Institute of Mathematical Sciences \& Department of Mathematics,

The Chinese University of Hong Kong, Shatin, N.T., Hong Kong

E-mail address: qyuan103@link.cuhk.edu.hk

(Y. Yuan) South China Research Center for Applied Mathematics and InterdisciPlinary Studies, South China Normal University, Guangzhou, Guangdong, China

E-mail address: yyuan $2102 @ \mathrm{~m} . \mathrm{scnu} . \mathrm{edu} . \mathrm{cn}$ 